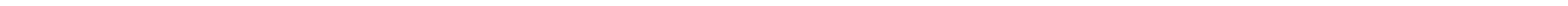


IMAGING VELOCITY AND ATTENUATION SCATTERERS WITH WAVE EQUATION MIGRATION 



\title{
IMAGING VELOCITY AND ATTENUATION SCATTERERS WITH WAVE EQUATION MIGRATION
}

\section{Proefschrift}

\author{
ter verkrijging van de graad van doctor \\ aan de Technische Universiteit Delft, \\ op gezag van de Rector Magnificus prof. ir. K.Ch.A.M. Luyben, \\ voorzitter van het College voor Promoties,
}

in het openbaar te verdedigen op vrijdag 12 november 2010 om 10:00 uur

door Bobby HAK

ingenieur in de technische wiskunde

geboren te Laren. 
Dit proefschrift is goedgekeurd door de promotor:

Prof. dr. W.A. Mulder

Samenstelling promotiecommissie:

Rector Magnificus, voorzitter

Prof. dr. W.A. Mulder, Technische Universiteit Delft, promotor

Prof. dr. ir. C.P.A. Wapenaar, Technische Universiteit Delft

Prof. dr. ir. R.J. Arts, $\quad$ Technische Universiteit Delft

Prof. dr. ir. C. Vuik, $\quad$ Technische Universiteit Delft

Prof. dr. D.G. Simons, Technische Universiteit Delft

Prof. dr. A.E.P. Veldman, Rijksuniversiteit Groningen

Dr. R.-E.A.M. Plessix, Shell International Exploration \& Production

\section{SUPPORT}

This work is part of the research programme of the 'Stichting voor Fundamenteel Onderzoek der Materie' (FOM), financially supported by the 'Nederlandse Organisatie voor Wetenschappelijk Onderzoek' (NWO) and the 'Stichting Shell Research'.

ISBN 978-90-9025799-0

Copyright (C) 2010 by Bobby Hak, Delft University of Technology, Delft, The Netherlands.

All rights reserved. No part of this book may be reproduced, in any form or by any means, without prior permission in writing from the author. clearpage 


\section{Contents}

$\begin{array}{ll}\text { Titlepage } & \text { i }\end{array}$

Contents $v$

Summary ix

Samenvatting $x i$

1 Introduction 1

1.1 Motivation ..................... . . 2

1.2 Thesis objectives . . . . . . . . . . . . . . . . 4

1.3 Thesis outline . . . . . . . . . . . . . . 4

2 Mathematical background 7

2.1 Wave equation . . . . . . . . . . . . 7

2.1.1 Stress-strain relation . . . . . . . . . . . . . 8

2.1.2 Low-loss approximation . . . . . . . . . . . . . . 12

2.1 .3 Constant quality factor . . . . . . . . . . . . . . 12

2.2 Migration . . . . . . . . . . . . . . . 13

2.2.1 Linearisation . . . . . . . . . . . . . . . . 14

2.2.2 Least-squares cost functional . . . . . . . . . . . . . 15

2.2 .3 Jacobian matrix . . . . . . . . . . . . . . . 16

2.2.4 Gradient and Hessian . . . . . . . . . . . . . 17

3 Non-uniqueness in iterative visco-acoustic migration 19

3.1 Introduction . . . . . . . . . . . . . . 20 
3.2 Governing equations . . . . . . . . . . . . . . 21

3.2.1 Constant density visco-acoustic wave equation . . . . 21

3.2.2 The Green function . . . . . . . . . . . . . . . . . . 22

3.2.3 Least-squares minimisation . . . . . . . . . . . . . 23

3.3 Synthetic example . . . . . . . . . . . . . . . . . 24

3.4 Gradient . . . . . . . . . . . . . . . . . . . 25

3.5 Preconditioner results . . . . . . . . . . . . . . . 28

3.6 Summary and conclusion . . . . . . . . . . . 32

4 An ambiguity in attenuation scattering imaging 33

4.1 Introduction . . . . . . . . . . . . . . . . . . . 34

4.2 Governing equations . . . . . . . . . . . . 35

4.3 3-D homogeneous background with 1-D scattering model . . 37

4.3.1 Scattered wavefield . . . . . . . . . . . . . . 37

4.3 .2 Ambiguity . . . . . . . . . . . . . 38

4.3 .3 Example . . . . . . . . . . . . . . . 42

4.3.4 Fourier representation . . . . . . . . . . . . . 44

4.3 .5 Migration . . . . . . . . . . . . . . . 47

4.4 2-D numerical example . . . . . . . . . . . . . . . . 48

4.5 Discussion . . . . . . . . . . . . . . . . 53

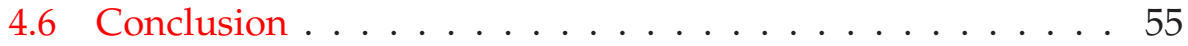

5 Migration for velocity and attenuation perturbations 57

5.1 Introduction . . . . . . . . . . . . . 58

5.2 Problem statement . . . . . . . . . . . . . . . 6 61

5.3 Resolution function for a constant model . . . . . . . . . 63

5.4 Other acquisition geometries . . . . . . . . . 68

5.4 .1 Circle . . . . . . . . . . . . . . . . . . . . . 69

5.4 .2 Cross-well data . . . . . . . . . . . . . . . . . . 69

5.4 .3 Diving waves . . . . . . . . . . . . . . 72

5.5 Discussion and conclusions . . . . . . . . . . . . 74

5.A Demigration for a 1D scattering model . . . . . . . . . 75

6 Seismic attenuation imaging with causality 77

6.1 Introduction . . . . . . . . . . . . . 78

6.2 Methodology . . . . . . . . . . . . . . . 80

6.2 .1 Forward modelling . . . . . . . . . . . . . . 80

6.2 .2 Linearised inversion . . . . . . . . . . . . . . 82 
6.2.3 Nonlinear inversion . . . . . . . . . . . . . . . . 83

6.3 Linearised inversion in constant background . . . . . . . . . 83

6.4 Nonlinear inversion . . . . . . . . . . . . . . . . . . . 87 87

6.4.1 Constant velocity model . . . . . . . . . . . . . . 87

6.4 .2 Noise . . . . . . . . . . . . . . . . . . . . . 88

6.4 .3 Marine model . . . . . . . . . . . . . . . . . . . 92

6.5 Conclusions . . . . . . . . . . . . . . . . . . 95

6.A Gradient of the least-squares functional . . . . . . . . 95

7 Conclusions and further research $\quad 99$

7.1 Conclusions . . . . . . . . . . . . . . . . . . 999

7.2 Suggestions for further research . . . . . . . . . . . 102

$\begin{array}{ll}\text { Bibliography } & 103\end{array}$

$\begin{array}{ll}\text { Acknowledgements } & 113\end{array}$

$\begin{array}{ll}\text { Curriculum Vitae } & 115\end{array}$ 


\section{Summary}

\section{Imaging velocity and attenuation scatterers with wave equation migration}

The thesis treats the seismic least-squares migration under the viscoacoustic assumption and in the frequency domain. The central question we try to answer in this thesis is: can we simultaneously retrieve velocity and attenuation perturbations? The attenuation may give more precise fluid information and can, for instance, contribute in making a distinction between a gas- or fluid-filled reservoir. The benefit for exploration geophysicists is obvious.

After a general introduction, we present the physical and mathematical background and focus on the visco-acoustic wave equation and the leastsquares formulation of the inverse problem. The thesis revolves around the four chapters thereafter, from which two were, and one is about to be published in established geophysical journals. These chapters describe the development from inversion based on a simple but non-causal wave propagation model to the proper causal approach.

In the frequency domain, including attenuation amounts to using a complex-valued wave velocity. In the third chapter, we considered several preconditioners with aim to not only speed up the convergence of the conjugate-gradient method, which is used to solve the linear system of the inverse problem, but also to compensate for the crosstalk -the interferance of one parameter on the other parameter- that appears when updating the velocity and attenuation perturbations simultaneously. None of the preconditioners enabled us to accurately reconstruct the perturbations. It 
turns out that the result is not unique and also didn't correspond to the true perturbations in any of the examples considered. We conclude that perturbations of the velocity and the attenuation cannot be reliably retrieved. A more fundamental problem must lie at the root of this problem.

In the fourth chapter, we derive two models that give practically the same seismic responses. This reveals that there is a non-uniqueness or ambiguity in the inverse problem that already begins at the forward modelling stage.

The fifth chapter investigates the influence of the seismic acquisition geometry on the ambiguity. We examine iterative migration results of synthetic data sets for different types of acquisitions. A circular acquisition leads to a correct reconstruction of both the velocity and attenuation perturbations. In case of an acquisition that is more realistic for explorationists, i.e., a surface or cross-well acquisition, the reconstructions are still significantly affected by the crosstalk and the ambiguity remains. Also in a setting where the background velocity increases linearly with depth -illuminating the perturbation from above but also partly from below-the ambiguity remains.

These chapters are all based on a non-causal attenuation model, which is not all that realistic. Also, the synthetic examples used so far are mostly very simple and free of noise. Therefore, we consider a more realistic model in the sixth chapter by accounting for the frequency-dependence of the real part of the velocity with the classic Kolsky-Futterman formula. We also introduce slightly more complex subsurface models. We carry out several linear and non-linear iterative migrations and show that the ambiguity is almost resolved, allowing us to more or less retrieve the velocity and attenuation perturbations. However, to achieve this result a large number of iterations is required to solve the least-squares inverse problem. This is because the gradient of the least-squares cost functional, which roughly correspond to a classic migration, contains substantial crosstalk.

Returning to the central question of this thesis, we conclude that we can only retrieve viscous scattering parameters if we account for dispersion by including causality. This requires data of sufficient bandwidth, as available in typical seismic datasets. 


\section{Samenvatting}

\section{Het afbeelden van snelheids- en dempings- verstrooiers met golfvergelijkingsmigratie}

Dit proefschrift behandelt kleinste-kwadraten migratie van seismische date onder de viskeus-akoestische aanname en in het frequentie domein. De centrale vraag die we proberen te beantwoorden in dit proefschrift is: kunnen we gelijktijdig verstoringen in de snelheid en attenuatie terugvinden? Attenuatie kan meer precieze informatie geven over de aanwezigheid van een vloeistof of van gas. Ter illustratie, het draagt bij in het maken van nderscheid tussen een reservoir dat gevuld is met gas of vloeistof. Het voordeel voor de exploratie geofysicus is evident.

$\mathrm{Na}$ een algemene introductie presenteren we de fysische en wiskundige achtergrond en richten ons op de viskeus-akoestische golfvergelijking en het formuleren van het kleinste-kwadraten inversieprobleem. Het proefschrift is gebaseerd op de vier hoofdstukken die volgen, van welke twee zijn, en één binnenkort wordt, gepubliceerd in gerenomeerde geofysische tijdschriften. Deze hoofdstukken beschrijven de ontwikkeling van de inversie van een simpele, maar niet-causale golfvoortplantingsmodel tot een nette causale aanpak.

In het frequentie-domein betekent het gebruik van demping dat een complexwaardige golfsnelheid nodig is. In het derde hoofdstuk behandelen we verschillende preconditioners voor de iteratieve migratie. Het doel is niet alleen de convergentie van de geconjugeerde-gradienten methode te versnellen, maar ook om te compenseren voor overspraak -invloed van de ene parameter op de andere parameter- die optreedt wanneer de 
snelheids- en dempingsverstrooiers gelijktijdig worden bijgewerkt. Geen van de preconditioners helpt ons om accuraat de verstrooiers terug te vinden. Het blijkt dat de resultaten niet uniek zijn en ook in geen enkel geval overeen komen met de werkelijke verstrooier. Wij concluderen dat de verstrooiers ten gevolge van snelheids- en dempingsverstoringen niet met zekerheid bepaald kunnen worden en dat er een meer fundamenteel probleem aan de wortel van dit probleem moet liggen.

In het vierde hoofdstuk leiden we twee modellen af die praktisch dezelfde seismische data geven. Dit laat zien dat er geen uniciteit maar een ambiguiteit is in het inverse probleem die al begint op het niveau van de voorwaardse modellering.

Het vijfde hoofdstuk onderzoekt de invloed van de geometrie van de seismische acquisitie op de ambiguïteit. We bestuderen resultaten van iteratieve migratie van synthetische data voor verschillende acquisities. Een circelvormige acquisitie leidt tot een correcte reconstructie van zowel de snelheids- als dempingsverstrooiers. In het geval van een acquisitie die meer realistisch is voor seismische exploratie, d.w.z. een acquisitie aan het oppervlak of tussen twee putten, wordt de reconstructie nog erg beïnvloed door de overspraak en blijft de ambiguïteit. Ook wanneer de snelheids van het achtergrondmodel lineair toeneemt met diepte-waardoor de verstrooier van boven, maar ook deels van beneden wordt belicht- blijft de ambiguïteit.

Al deze hoofdstukken zijn gebaseerd op een niet-causaal dempingsmodel, wat niet heel erg realistisch is. Bovendien zijn synthetische voorbeelden veelal erg simpel en zonder ruis. Daarom gebruiken we een meer realistisch model in het zesde hoofdstuk door rekening te houden met de frequentie-afhankelijkheid van het reëelwaardige deel van de snelheid, zoals gegeven door de klassieke Kolky-Futterman formule. Ook introduceren we een iets ingewikkelder model van de ondergrond. We doen verschillende lineaire en niet-lineaire iteratieve migraties en laten zien dat de ambiguïteit bijna verholpen wordt en ons toestaat om de snelheidsen dempingsverstrooiing min of meer te bepalen. Desalniettemin, om dit resultaat te behalen is een groot aantal iteraties nodig voor het oplossen van het kleinste-kwadraten inversieprobleem. Dit is omdat de gradient van de kleinste-kwadraten kosten functionaal, die nagenoeg overeenkomt me de klassieke migratie, substansiële overspraak bevat.

Terugkomend op de centrale vraag van dit proefschrift, concluderen we dat we alleen de viskeuze parameters kunnen terugvinden als we rekening 
houden met dispersie door middel van causaliteit. Hiervoor is data nodig dat voldoende bandbreedte heeft, zoals die beschikbaar is in een typische seismische dataset. 


\section{Chapter 1}

\section{Introduction}

In geophysics we study the Earth by quantitative physical methods, especially by seismic reflection and diffraction, gravity, magnetic, electrical, electromagnetic, and radioactivity methods (Sheriff, 2002). In applied geophysics, or exploration geophysics, these methods are employed in the search for oil, gas, minerals, water, etc., with the objective of economic exploitation. To achieve this goal, several imaging techniques have been developed since the mid $20^{\text {th }}$ century.

The imaging techniques aim to transform seismic data recorded as a function of arrival time into the features that produced the record (Sheriff, 2002). It involves focussing and positioning. The model produced by the imaging technique gives the geometry of geologic features and, ideally, corresponding physical properties such as seismic velocity, density, porosity, and attenuation. Seismic sources generate waves that travel through the medium and may be recorded by a detector. The recorded data consist of all sorts of waves, for instance, scattered waves that have been reflected because of impedance -the product of density and wave velocity-contrasts in the medium.

Migration is an imaging technique involving rearrangement of seismic recorded data such that reflections and diffractions are placed at their true locations in time or depth. In the early 1980s, Lailly (1983) and Tarantola (1984) recognised that the migration step not only relocated reflections and diffractions to their original location, but also maintained a relative amplitude such that medium parameters could be reconstructed. 

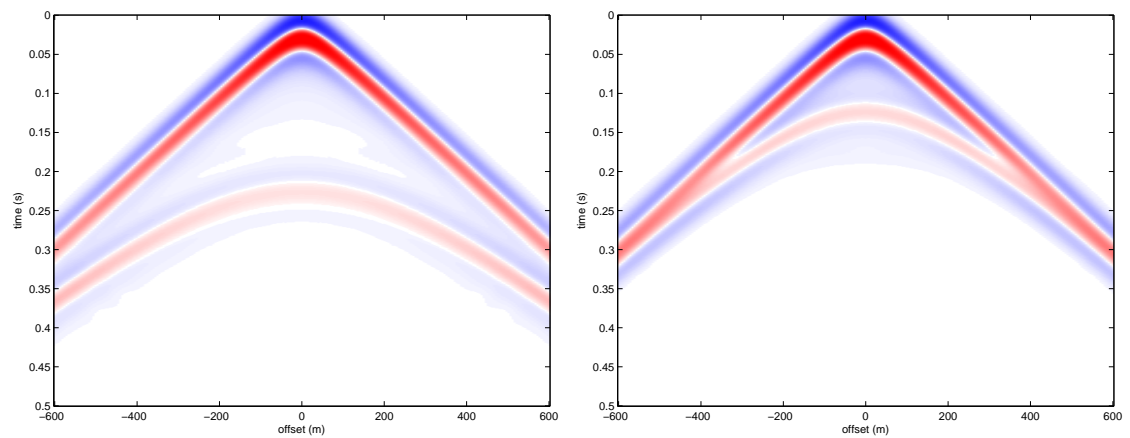

Figure 1.1: Recorded data for model 1 (left panel) and model 2 (right panel) as described by Vasheghani and Lines (2009). In model 1 the wave velocity and quality factor change simultaneously. In model 2 an additional layer is added, of which only the quality factor has changed. Data are computed with a frequency domain code on a $1200 \times 1200$ grid using a Ricker wavelet source with peak frequency at $18 \mathrm{~Hz}$. One source is placed in the middle of the domain at $200 \mathrm{~m}$ depth. Receivers are placed every $5 \mathrm{~m}$ at $150 \mathrm{~m}$ depth.

This opened a way to do a true-amplitude migration by a method that iteratively formed the true value of the medium parameter(s).

There is a wide range of migration algorithms (e.g., dip moveout, raytrace, Kirchhoff, and wave-equation, time- and frequency-domain). Here, when we talk about migration, we refer to wave-equation migration in the frequency domain.

\subsection{Motivation}

By now, for acoustic migration most fundamental problems are reasonably well understood (Mulder and Plessix, 2004; Yoon et al., 2004). An acoustic model assumes the Earth behaves as a fluid where the waves that propagate through the medium are pressure waves (also called P-waves). At interfaces, the P-impedance contrast gives reflections and refractions. However, the acoustic representation of the Earth is not realistic. A more representative description, although still far from the truth, is a viscoacoustic model that includes wave attenuation.

Waves lose their energy while travelling through the Earth, not only 


\begin{tabular}{lcccc}
\hline \multicolumn{2}{c}{ Thickness (m) } & V (m/s) & \multicolumn{2}{c}{ Quality factor } \\
& & & model 1 & model 2 \\
\hline \hline Layer 1 & 300 & 2000 & 40 & 40 \\
Layer 2 & 100 & 2000 & 40 & 3 \\
Layer 3 & 800 & 3000 & 800 & 800 \\
\hline
\end{tabular}

Table 1.1: Parameters used in models 1 and 2 for synthetic forward modeling of surface seismic experiments.

because of geometrical spreading, but also by conversion into heat, or possibly mode conversions, for example. A part of these attenuating effects are due to viscosity of the medium. A viscous medium not only decreases the amplitude, but can also cause phase changes. The viscosity may be described by an Earth's quality factor. In the frequency-domain, it can be expressed by a complex-valued wave velocity, resulting in complex-valued impedance contrasts. The Earth's quality factor may contain information about fluid saturation, which can distinguish between a gas-filled or fluidfilled reservoir, for instance. For seismic exploration, the relevance of knowing its value is obvious.

For the complex-valued impedance, it can be shown that both the real and imaginary part of the impedance contrasts produce reflections and refractions. For an acoustic medium only the real part produces these. Consequently, assuming a visco-acoustic medium gives different recordings than assuming an acoustic medium. Figure 1.1 shows an example, similar to the one given by Vasheghani and Lines (2009), for two models given by the parameters of Table 1.1.

Although the example is quite extreme, it shows that the data are indeed influenced by the viscous effects. Moreover, explaining such data by an acoustic model, rather than a visco-acoustic model, will place its reflection point at the wrong time or depth position.

As was said earlier, in the frequency domain a complex-valued model parameter should be considered when including viscous effects. In fact, for migration the whole algorithm becomes complex-valued, whereas in a lossless medium only the real part is needed. Luckily, the additional computational cost is relatively small in 2D with a frequency-domain code.

Hence, from realistic, economical, and computational point of view, and maybe even because of necessity, it is plausible to include viscosity. The 
question is now if iterative migration can recover small-scale perturbations in the visco-acoustic parameters.

We started by investigating this question assuming a constant density, leading to the constant-density visco-acoustic wave equation or viscoscalar wave equation. It proved to be an opening to an interesting area of research and it led to several noteworthy observations which we will present in this thesis.

\subsection{Thesis objectives}

A propagating wave in a visco-acoustic medium loses its energy -and with that its wave amplitude- due to viscous effects. Compared to an acoustic medium, the recorded data of a visco-acoustic medium will thus have smaller amplitudes. In addition, the phase of the waves will be distorted. These effects are illustrated in the work of Lines et al. (2008) and Vasheghani and Lines (2009), for instance. The recorded data can be significantly different by taking into account changes in viscosity (Figure 1.1). As a result, data can only be explained correctly when viscous effects are taken into account. Moreover, including viscous effects is more realistic than ignoring them. Therefore, we wish to include viscosity in our migration algorithm.

The governing parameters then depend on a wave velocity for a lossless medium and an Earth quality factor, for instance. Estimating these governing parameters did receive attention from several authors, but results vary (Ribodetti and Virieux, 1998; Causse et al., 1999; Hicks and Pratt, 2001) and many of them report it is difficult to obtain good, reliable results. In this thesis, we aim to show why and when imaging of simultaneous visco-acoustic parameters is difficult and investigate what we can do to overcome this difficulty.

\subsection{Thesis outline}

The thesis is built up as follows. The second chapter gives a mathematical foundation that is used in the remainder of this thesis. There, elementary steps for the derivation of the constant-density visco-acoustic wave equation are given. Linear viscosity is included via the stress-strain relation 
for a Kelvin-Voigt model. Then, equations for wave-equation migration are given, where we assume the Born approximation is valid. In the third chapter we will show that (iterative) migration of complex-valued parameters results in erroneous behaviour; despite convergence of the algorithm, different preconditioners give different results, and, on top of that, none of the schemes gives the correct amplitude for both the real and imaginary part of the parameter. We show that the gradient that should update the parameter update, initially does more harm than good. In the fourth chapter we show non-uniqueness for a layered medium if no additional constraints on the parameters are assumed. We show that this ambiguity is already visible in the recorded data. The explanation is accompanied by a synthetic data example. This chapter is also available as journal article (Mulder and Hak, 2009). In the fifth chapter we will investigate different acquisition geometries and their influence on the occurrence of the ambiguity. We review an acquisition that is circular around the scatterer, a cross-well acquisition, and a setting in which we assume the velocity of the background model increases linearly with depth. A journal article of this chapter is available (Hak and Mulder, 2010a). In the sixth chapter we elaborate upon the previous chapter. Here, we investigate if the ambiguity shows up when causality is included in the scatterer model, when non-linear effects without causality are taken into account, or both. The chapter includes an example that has noise and a more realistic synthetic marine example. It has been submitted as a journal article (Hak and Mulder, 2010b). The seventh chapter summarises the main findings. 


\section{Chapter 2}

\section{Mathematical background}

In this chapter, we review the mathematical background of this thesis. Most of the basic mathematical concepts that are used in later chapters will be presented here. Further details on these concepts, can be found in many standard textbooks, for instance Aki and Richards (2002).

Section 2.1 contains the elementary steps in the derivation of the wave equation and the viscous parameter is discussed. The specific type of wave equation depends on the stress-strain relation that is used and the wavefield the equations are expressed in. In Section 2.2 the wave equation is linearised under the Born approximation. Expressions are given for the error, the gradient, and the Hessian of a least-squares cost functional. Here, we will present the linear theory that is valid when displacements or deformations are small. Distinction between time-domain and frequencydomain parameters is made by using lower-case and upper-case symbols, respectively. Operators are in an upright font and the distinction between time and frequency-domain is indicated by capitalising the latter. Equations will be in index-notation and we adopt Einstein's summation convention for repeated subscripts.

\subsection{Wave equation}

In this section, we derive the constant-density visco-acoustic wave equation, or visco-scalar wave equation. We start with formulating a linear 
stress-strain relation for solids that gives the traction forces on a closed volume, where it is assumed that deformations are small. For fluids, the shear stresses vanish and therefore the stress-strain relation is simplified in the next step. Then, a general wave equation is found by balancing the forces that work on the closed system with Newton's second law. By expressing the wave equation in a pressure wavefield rather than a displacement wavefield, we obtain the desired visco-scalar wave equation. Last, some more words are devoted to the viscous parameters, which plays an important role in this thesis.

\subsubsection{Stress-strain relation}

The common theory assumes stresses and strains are connected through a linear relation. Stress is a measure for the average amount of force exerted per unit area of a surface within a deformable body on which internal forces act. The internal forces are the consequence of deformations in the medium. Deformation is expressed by strains, which are a measure for the change of dimensions or shape caused by stress.

When the wave is far away from the source, the deformations of a body are relatively small, justifying a low order approximation of the deformation. The first order approximation of the relation between strains $\varepsilon$ and displacements $u$ is given by

$$
\varepsilon_{k l}=\frac{1}{2}\left(\partial_{k} u_{l}+\partial_{l} u_{k}\right) .
$$

A linear relation connecting stresses $\tau$ and strains can be expressed using an operator $\Psi$. This operator maps the strains to stresses, and in many cases can be seen as a function. However, operators may also contain derivatives or integrals, for instance. The stress-strain relation has 81 independent elements, which by themselves may be operators. In index notation this can be written as,

$$
\tau_{i j}=\Psi_{i j k l} \varepsilon_{k l} .
$$

Here, each operator $\Psi_{i j k l}$ can contain (time) derivatives and may depend on the strain history. The operator should be causal, assuring that no stress is present before zero time. Because both the strain and stress are symmetrical and we assume they have a linear relation, the elements of $\Psi$ 
also have symmetries. In addition, a thermodynamic argument imposes symmetry as well, such that we have

$$
\Psi_{i j k l}=\Psi_{k l i j}=\Psi_{j i k l}=\Psi_{i j l k} .
$$

Hence, the total amount of independent operators can be reduced to 21, which in the isotropic case will even further reduce to just two independent operators. For the isotropic case, it is assumed the medium has the same physical properties regardless of the direction it is measured in. As a result, the operators are given by

$$
\Psi_{i j k l}=\delta_{i j} \delta_{k l} \mathrm{M}+\left(\delta_{i k} \delta_{j l}+\delta_{i l} \delta_{j k}\right) \mathrm{G},
$$

where $\delta$ is the Kronecker delta and we see the two independent operators are $\mathrm{M}$ and $\mathrm{G}$.

A well-known stress-strain relation is given by Hooke's law, which results in the elastic stress-strain relation. There, we have

$$
\mathrm{M} \varepsilon_{k l}=\lambda \varepsilon_{k l}, \quad \text { and } \quad \mathrm{G} \varepsilon_{k l}=\mu \varepsilon_{k l} \text {. }
$$

and we see that applying the operators $M$ and $G$ to the strain function gives a multiplication with $\lambda$ and $\mu$, respectively, such that we find

$$
\tau_{i j}=\delta_{i j} \lambda \varepsilon_{k k}+2 \mu \varepsilon_{i j} .
$$

Parameters $\lambda$ and $\mu$ are known as the Lamé parameters. When the second term vanishes, i.e., when $\mu=0$, this leaves the acoustic relation.

There are several stress-strain relations that include viscosity, e.g., the Standard Linear Solid model, the Maxwell model, and the Kelvin-Voigt model. All relations aim to model the time-depending behaviour of the wave, changing the form of the $\mathrm{M}$ and $\mathrm{G}$, often including time derivatives. For a visco-acoustic medium $\mathrm{G}$ equals zero and we have

$$
\tau_{11}=\tau_{22}=\tau_{33}=\mathrm{M} \varepsilon_{k k} .
$$

All other stresses vanish. 


\section{Newton's second law}

Newton's second law relates forces and velocity, yielding conservation of linear momentum for a closed system within a volume $\Omega$ with boundary $\Gamma$. It states the total change of momentum of a body is equal to the total force on the body and the traction force acting normal to the surface of the body. Hence,

$$
\int_{\Omega} \rho \ddot{u} \mathrm{~d} \Omega=\int_{\Omega} \rho f \mathrm{~d} \Omega+\int_{\Gamma} t(\hat{n}) \mathrm{d} \Gamma .
$$

The density is denoted by $\rho$, the body force per unit mass is given by $f$, and the traction is given by $\boldsymbol{t}(\hat{n})=\tau \cdot \hat{n}$, where $\hat{n}$ is the normal vector. By applying Gauss' theorem to the surface integral, we find Cauchy's first law of motion

$$
\rho \ddot{u}=\rho f+\nabla \cdot \tau .
$$

The wave equation can now be obtained by defining a stress-strain relation for this medium and using equation (2.1).

\section{Visco-acoustic wave equation}

Suppose the operator $M$ can be represented by a convolution with the function $m(t, x)$ in the time-domain or multiplication with the function $M(\omega, x)$ in the frequency-domain. For the isotropic case and a Kelvin-Voigt medium, the wave equation and its frequency-domain representation are found by combining equation (2.7) and (2.9), which gives

$$
\rho \ddot{\boldsymbol{u}}=\nabla(m *(\nabla \cdot \boldsymbol{u}))+\rho \boldsymbol{f}, \quad \text { and } \quad \omega^{2} \rho \boldsymbol{U}+\nabla(M(\nabla \cdot \boldsymbol{U}))=-\rho \boldsymbol{F},
$$

respectively. Now assume the density is constant. By taking the divergence on both sides of the equality sign and defining the pressure wavefield $P=-M(\nabla \cdot \boldsymbol{U})$ and source function $S=-\rho(\nabla \cdot \boldsymbol{F})$ it follows that

$$
-\frac{\omega^{2}}{v^{2}} P-\nabla^{2} P=S, \quad v=\sqrt{\frac{M}{\rho}} .
$$


This is the constant-density visco-acoustic wave equation or scalar wave equation, which is one of the central equations in this thesis. The wave velocity of the visco-acoustic medium $v(\omega, x)$ may depend on frequency and position and may be complex valued.

Issues that remain are the definition of the parameter $M$ and inclusion of causality in the frequency-domain. In the next paragraph, we go deeper into this viscous parameter and causality issue. First, we define a more commonly used viscous parameter, after which we will include the causality constraints.

\section{Viscous parameter}

The intrinsic quality factor, or Earth quality factor, measures the energy dissipated from the system. O'Connell and Budiansky (1978) defined this term, denoted by $Q$, as

$$
\frac{4 \pi}{Q}=-\frac{\Delta \phi}{\langle E\rangle},
$$

where $-\Delta \phi$ is the energy dissipation per stress cycle and $\langle E\rangle$ the average energy per cycle. Suppose now, we have a single frequency component of the stress at which $\tau(t)=\tau_{0} e^{\mathrm{i} \omega t}$. The energy and dissipation energy of a system with stress was derived by Bland (1960) as

$$
\begin{aligned}
E(t) & =\frac{1}{4} M_{r}\left|\tau_{0}\right|^{2}+\frac{1}{4} \operatorname{Re}\left\{\left(M(\omega)-\omega M^{\prime}(\omega)\right) \tau_{0}^{2} e^{2 \mathrm{i} \omega t}\right\}, \\
\delta E(t) & =\frac{1}{2} M_{i}\left|\tau_{0}\right|^{2}-\frac{1}{2} \omega^{2} \operatorname{Re}\left\{M^{\prime}(\omega) \tau_{0}^{2} e^{2 \mathrm{i} \omega t}\right\},
\end{aligned}
$$

where $M=M_{r}+\mathrm{i} M_{i}$ is the frequency-domain kernel of the operator relating stresses to strains. From this, for this single frequency, the energy dissipation, average energy, and quality factor are then given by

$$
\langle E\rangle=\frac{1}{4} M_{r}\left|\tau_{0}\right|^{2}, \quad \Delta \phi=\pi M_{i}\left|\tau_{0}\right|^{2}, \quad Q=-\frac{M_{r}}{M_{i}} .
$$

Note that when we consider all frequencies, the quality factor is frequency dependent. 


\subsubsection{Low-loss approximation}

For a wave that is damped the wavenumber $k(\omega)$ can be defined as

$$
k=\frac{\omega}{c}+\mathrm{i} \alpha .
$$

where $c(\omega)$ is the wave phase velocity and $\alpha(\omega)$ is the attenuation coefficient. For a constant-density, the visco-acoustic wave equation, equation (2.11), also yields a wavenumber, that is given by

$$
k^{2}=\frac{\omega^{2} \rho}{M}=\frac{\omega^{2} \rho}{M_{r}\left(1-\mathrm{i} Q^{-1}\right)} \simeq \frac{\omega^{2} \rho}{M_{r}}\left(1+\mathrm{i} Q^{-1}\right) .
$$

The real part of $k$ is positive and we assumed $Q \gg 1$, meaning the losses of this system are low. In that case, it follows that the phase velocity and attenuation may be given by

$$
c \simeq \sqrt{\frac{M_{r}}{\rho}}, \quad \text { and } \quad \alpha \simeq \frac{\omega}{2 Q c} .
$$

For causality to hold, the real and imaginary part of $M(\omega)$ should fulfill the Kramer-Krönig relation.

\subsubsection{Constant quality factor}

The stress-strain relation is a causal relation, meaning no stress is present before a strain is applied and visa-versa. It was proven by Titchmarsh (1948) that if a system is causal, the Kramer-Krönig relations should hold. Here, the Kramer-Krönig equations relate real and imaginary part of the complex modulus $M$ by a Hilbert transform, i.e.,

$$
M_{r}(\omega)=\frac{2}{\pi} \mathcal{P} \int_{0}^{\infty} \frac{\omega^{\prime} M_{i}\left(\omega^{\prime}\right)}{\omega^{\prime 2}-\omega^{2}} \mathrm{~d} \omega^{\prime}, \quad \text { and } \quad M_{i}(\omega)=-\frac{2 \omega}{\pi} \mathcal{P} \int_{0}^{\infty} \frac{M_{r}\left(\omega^{\prime}\right)}{\omega^{\prime 2}-\omega^{2}} \mathrm{~d} \omega^{\prime},
$$

where $\mathcal{P}$ denotes that Cauchy's principle value should be taken. The Kramer-Krönig relations lead to the approximation (see Aki and Richards, 
2002, box 5.8):

$$
\frac{\omega}{c(\omega)}=\frac{\omega}{c_{\infty}}+\mathcal{H}[\alpha(\omega)]
$$

where $c_{\infty}$ is the limit of $c(\omega)$ as $\omega \rightarrow \infty$, and $\mathcal{H}[\alpha(\omega)]$ denotes the Hilbert transform of the attenuation factor $\alpha$. It can be shown this condition only holds for a frequency-dependent quality factor $Q$.

For parameter estimation it is more convenient to have a constant quality factor. The most commonly used model in seismic exploration is the Kolsky-Futterman model (Kolsky, 1956; Futterman, 1962), which was used by Aki and Richards. Following their derivation, the visco-acoustic wave velocity $v$, for a quality factor that is constant over the frequency band, is approximately given by

$$
v \approx c\left[1+\frac{1}{\pi Q} \log \left(\frac{\omega}{\omega_{0}}\right)-\mathrm{i} \frac{1}{2 Q}\right],
$$

or its equivalent

$$
\frac{1}{v} \approx \frac{1}{c}\left[1-\frac{1}{\pi Q} \log \left(\frac{\omega}{\omega_{0}}\right)+\mathrm{i} \frac{1}{2 Q}\right], \quad \text { and } \quad \frac{1}{v^{2}} \approx \frac{1}{c^{2}}\left[1-\frac{2}{\pi Q} \log \left(\frac{\omega}{\omega_{0}}\right)+\mathrm{i} \frac{1}{Q}\right] .
$$

The angular frequency $\omega_{0}=2 \pi f_{0}$ is a reference frequency against which causality is checked, which commonly is chosen as $f_{0}=1 \mathrm{~Hz}$. Note that despite its popularity, the Kolsky-Futterman model only approximately fulfills the Kramer-Krönig relations.

A more simple equation is the model by Toksöz and Johnston (1981), that does not include the causality correction and reads

$$
\frac{1}{v^{2}} \approx \frac{1}{c^{2}}\left[1+\mathrm{i} \frac{1}{Q}\right]
$$

\subsection{Migration}

Migration is a step in seismic processing in which reflections in seismic data are moved to their correct locations in space or time. These locations may 
differ from the recorded time image in areas where there are significant and rapid lateral or vertical changes of the wave velocity.

In wave equation migration, this is accomplished by applying the wave equation for a specific Earth model to the recorded seismic data and correlating that with modelled source data. Hence, the method requires and depends on a velocity model given by this Earth model. The resulting image shows the reflectors at their true location in this Earth model, but may also contain artefacts.

In the early 1980s, Lailly (1983) and Tarantola (1984) showed that the gradient of the least-squares cost functional with respect to the model parameter(s) provides a migration image that maintains relative amplitudes. This opens a way to iteratively reconstruct the parameter amplitude using a gradient-based method. In addition, (an approximation of) the Hessian may provide additional information, giving a better step length to take in the direction of the gradient, and, in general, also adjusting the direction. The Hessian is the second derivative of the least-squares cost functional with respect to the model parameter(s). In this section, first the wave equation is linearised with the Born approximation, after which analytic expressions are given for the gradient and Hessian. The equations in this paragraph are presented for vector functions, although for the constantdentsity visco-acoustic wave-equation, a scalar representation would be sufficient.

\subsubsection{Linearisation}

The wave equation is linearised in the model parameters, such that variation in the wavefield becomes independent of perturbations of the parameters. The wave equation, like equation (2.9), can be put in the form:

$$
\llcorner u=-f,
$$

where the model operator $L$ may depend on model parameters $v$. The operator works on the field $u$, which is excited by a source function $f$. Assume now the operator is linear in the parameter $\boldsymbol{v}$. Then, the parameters can be written by the sum of a background parameter vector $\boldsymbol{v}_{b}$, giving a background operator $L_{b}$, and a vector of much smaller variations around this background $\delta \boldsymbol{v}$, giving a scatterer operator $\delta \mathrm{L}$. Similar, the wavefield $\boldsymbol{u}$ may be written as a sum of a background field $\boldsymbol{u}_{b}$ that solves 
for the background model, and a scattered field $\delta \boldsymbol{u}$ that explains the small perturbations about this background field. In this case, equation (2.23) may be written as

$$
\left(\mathrm{L}_{b}+\delta \mathrm{L}\right)\left(\boldsymbol{u}_{b}+\delta \boldsymbol{u}\right)=-f,
$$

Note that both $L_{b}$ and $\delta L$ are also linear in their parameter.

In the Born approximation, it is assumed that the reflection data of the scattered wavefield are much weaker than the data of the incident wavefield, such that they may be neglected. By dropping the term containing the reflection data of scattered wavefield, the wave equation splits into the pair of equations

$$
\mathrm{L}_{b} \boldsymbol{u}_{b}=-f, \quad \mathrm{~L}_{b} \delta \boldsymbol{u}=-\delta\left\llcorner\boldsymbol{u}_{b} .\right.
$$

The background model should not contain significant reflectors in the seismic frequency bandwidth. Assume Green's tensor $\mathcal{G}$ solves

$$
\mathrm{L}_{b} \mathcal{G}\left(\omega, x_{\mathrm{s}}, x\right)=-\mathbf{I} \delta\left(x-x_{\mathrm{s}}\right),
$$

where I is the identity matrix of appropriate size. Green's tensor gives the wavefield at $x$ in each direction due to a delta function perturbation at $x_{\mathrm{s}}$ in those directions, or vica versa. For a delta-function source at position $x_{\mathrm{s}}$, having a source wavelet given by $w_{i}(\omega)$, the scattered wavefield is given by

$$
\delta \boldsymbol{u}\left(\omega, x, x_{\mathrm{s}}\right)=\int_{\Omega} \mathrm{d} \xi \mathcal{G}(\omega, \xi, x) \delta \mathrm{L} \mathcal{G}\left(\omega, x_{\mathrm{s}}, \xi\right) w(\omega) .
$$

where the scattered operator $\delta \mathrm{L}$ is evaluated at $\xi$.

\subsubsection{Least-squares cost functional}

The error between simulated and measured data for a specific source $x_{\mathrm{s}}$, receiver $x_{\mathrm{r}}$, and frequency $\omega$ can be expressed by an error $\epsilon$. For the scattered model, define the error as

$$
\boldsymbol{\epsilon}\left(\omega, x_{\mathrm{s}}, x_{\mathrm{r}}\right)=\int_{\Omega} \mathrm{d} x \mathrm{R}_{\mathrm{r}(\mathrm{s})} \delta \boldsymbol{u}\left(\omega, x_{\mathrm{s}}, x\right)-\delta \boldsymbol{u}^{\mathrm{obs}}\left(\omega, x_{\mathrm{s}}, x_{\mathrm{r}}\right) .
$$


Here $R_{r(s)}$ is a mapping operator restricting the wavefield to receiver locations and we corrected the observed data, such that $\delta \boldsymbol{u}^{\text {obs }}$ contains only the scattered data. The least-squares cost functional $\mathcal{J}$ for the linearised equations can now be defined as

$$
\mathcal{J}=\sum_{\mathrm{r}(\mathrm{s}), \mathrm{s}} \int_{\mathcal{W}} \mathrm{d} \omega\left|\epsilon\left(\omega, x_{\mathrm{s}}, x_{\mathrm{r}}\right)\right|^{2},
$$

where we sum the error over all sources and receivers, indexed by s and $\mathrm{r}(\mathrm{s})$, respectively and integrate over the frequency bandwidth defined by the domain $\mathcal{W}$. The model perturbations $\delta \boldsymbol{v}$ are optimised to obtain the smallest difference between simulated and observed data, where the wavefield is subjected to the specific wave equation.

\subsubsection{Jacobian matrix}

For further use, here we define the Jacobian matrix $\mathfrak{g}$ with the first order derivatives of the displacement vector with respect to parameters vector, yielding

$$
\mathfrak{g}=\frac{\partial \delta \boldsymbol{u}}{\partial \delta \boldsymbol{v}}, \quad \text { where, } \quad \mathfrak{g}_{k}=\frac{\partial \delta \boldsymbol{u}}{\partial \delta v_{k}}, \quad \text { and } \quad \mathfrak{g}_{i k}=\frac{\partial \delta u_{i}}{\partial \delta v_{k}} .
$$

The colomns of the matrix are denoted by $\mathfrak{g}_{k}$, giving the derivative of the displacement vector with respect to a single parameter. Its elements are denoted by $\mathfrak{g}_{i k}$, representing a single partial derivative. From the Born approximation it follows the background wavefield is independent of the scattered parameters and thus, for a given background model $L_{b}$, that

$$
\mathrm{L}_{b} \mathfrak{g}_{k}=\frac{\partial \delta \mathrm{L}}{\partial \delta v_{k}} \boldsymbol{u}_{b} .
$$

In terms of Green's tensors this means for a point-scatterer of parameter $\delta v_{k}$ at location $x_{\mathrm{q}}$ that

$$
\mathfrak{g}_{k}\left(x, x_{\mathrm{q}}, x_{\mathrm{s}}, \omega\right)=\mathcal{G}\left(\omega, x_{\mathrm{q}}, x\right) \frac{\partial \delta \mathrm{L}}{\partial \delta v_{k}} \mathcal{G}\left(\omega, x_{\mathrm{s}}, x_{\mathrm{q}}\right) w(\omega),
$$


where the derivative of the operator is evaluated in the point $x_{\mathrm{q}}$. Recall that at the beginning of section 2.2.1, we assumed the model operator was linear in its parameter. Clearly, the derivative of the error then becomes

$$
\frac{\partial \epsilon}{\partial \delta v}=\int_{\Omega} \mathrm{d} x \mathrm{R}_{\mathrm{r}(\mathrm{s})} \frac{\partial \delta \boldsymbol{u}}{\partial \delta \boldsymbol{v}}=\int_{\Omega} \mathrm{d} x \mathrm{R}_{\mathrm{r}(\mathrm{s})} \mathfrak{g}\left(\omega, x, x_{\mathrm{q}}, x_{\mathrm{s}}\right)
$$

\subsubsection{Gradient and Hessian}

The gradient is the first derivative of the cost functional with respect to the model parameters in some point. For a given estimate of the perturbed model parameters, the corresponding scattered field can be obtained, where the gradient is given by

$$
g\left(x_{\mathrm{q}}\right)=\left(\frac{\partial \mathcal{J}}{\partial \delta \boldsymbol{v}}\right)^{\mathrm{H}}=\sum_{\mathrm{r}(\mathrm{s}), \mathrm{s}} \int_{\mathcal{W}} \mathrm{d} \omega\left[\frac{\partial \epsilon}{\partial \delta \boldsymbol{v}}\right]^{\mathrm{H}} \epsilon\left(\omega, x_{\mathrm{s}}, x_{\mathrm{r}}\right) .
$$

The gradient can easily be made dimensionless by pointwise scaling with the known background model. For the Hessian, or second derivative, we have

$$
H\left(x_{\mathrm{q}^{\prime}}^{\prime}, x_{\mathrm{q}}\right)=\frac{\partial}{\partial \delta \boldsymbol{v}^{\prime}}\left(\frac{\partial \mathcal{J}}{\partial \delta \boldsymbol{v}}\right)^{\mathrm{H}}=\sum_{\mathrm{r}(\mathrm{s}), \mathrm{s}} \int_{\mathcal{W}} \mathrm{d} \omega\left[\frac{\partial \epsilon}{\partial \delta \boldsymbol{v}}\right]^{\mathrm{H}}\left[\frac{\partial \boldsymbol{\epsilon}}{\partial \delta \boldsymbol{v}^{\prime}}\right] .
$$

Since also $\delta \mathrm{L}$ is linear in its parameters, the second derivative with respect to the model parameter vanishes. Here, a dimensionless Hessian matrix can be obtained by twice scaling with the background model.

It should be noted that computationally, the Hessian is out of reach for large-scale applications, although it is sometimes used for smaller sized problems (Pratt et al., 1998; Van Houten et al., 1999). However, a suitable diagonal approximation can already be sufficient in an iterative scheme. For the linearised acoustic case, this is shown by Shin et al. (2001) and Plessix and Mulder (2004), who mix results from the gradient computation with analytical estimates. For the elastic case Beylkin and Burridge $(1987,1990)$ show that the Hessian is blockdiagonal in the high-frequency asymptotic limit, as used in ray theory, if multi-pathing is avoided. Gélis et al. (2007) compute this blockdiagonal of the Hessian without approximations. 


\title{
Chapter 3
}

\section{Non-uniqueness in iterative visco-acoustic migration}

\begin{abstract}
We investigated iterative migration in the frequency domain for the constant density visco-acoustic wave equation. Then, impedance perturbations become perturbations of a complex-valued velocity. We used synthetic data generated by a single point scatterer consisting of both a velocity and an attenuation perturbation in a constant background model. The iterative solution scheme employed the conjugate gradient method, preconditioned by an approximation of the Hessian of the inverse problem. We found that different preconditioners not only affected the convergence rate but also led to different reconstructions of the scatterer. Also, none of the preconditioners could find a proper, band-limited reconstruction of the true perturbation. The results suggest that the migration problem has a non-uniqueness that goes beyond the usual one that is due to limitations in bandwidth of the recorded data and finite aperture of the acquisition. We conclude that there is a fundamental problem in getting reliable trueamplitude migration results for the constant density visco-acoustic wave equation.
\end{abstract}




\subsection{Introduction}

Seismic migration moves reflection events in the recorded data to their correct position in the subsurface. In addition, the migration algorithm maintains the relative amplitude behaviour of the reflectors. Lailly (1983) and Tarantola (1984) formulated migration as an inverse problem, relating the migration image to the gradient of the least-squares cost functional. This development initiated research on true-amplitude migration.

The migration algorithm follows from minimising a least-squares cost functional with respect to the model parameters. Minimising the discretised equations then amounts to solving a matrix-vector system, where the matrix is given by the Hessian and the right-hand side by the gradient of the cost functional with respect to the unknown model parameters. Theoretically, the system can be solved by multiplying the gradient with the inverse Hessian matrix. Unfortunately, for most realistic problems the computation of the Hessian and its inverse is too expensive, both in terms of storage size and computation time. Its full computation can be avoided by using an iterative scheme, whose convergence may be accelerated with an inexpensive approximation of the Hessian (Lambaré et al., 1992; Nemeth et al., 1999; Østmo et al., 2002; Plessix and Mulder, 2004).

The constant density visco-acoustic wave equation has the velocity and the quality factor as model parameters. The latter is directly related to attenuation, which describes the absorption of energy in the system. The amount of attenuation is affected by the saturation of fluids in the medium and may indicate the presence of hydrocarbons (Vasheghani and Lines, 2009). Several authors point out the benefit of estimating the quality factor (e.g., Causse et al., 1999; Hicks and Pratt, 2001; Ribodetti et al., 2007). Tarantola (1988) provided a theoretical background for inversion including attenuation. Attenuation has a damping effect on the wave amplitude. Recorded seismic data are always influenced by attenuation effects. Including attenuation into the propagation algorithm gives a better agreement with observed data. Also and more importantly, it opens the way to estimate its value with an inversion or migration scheme.

However, many authors acknowledge it is difficult to simultaneously find an accurate velocity model and a proper value for the quality factor (Tarantola, 1988; Ribodetti et al., 2007). For a visco-elastic medium, Tarantola recommends to update P-velocity, P-impedance, S-velocity, S-impedance, and attenuation, in that order. He concludes that finding 
attenuation is unlikely. Ribodetti et al. use visco-acoustic full-waveform tomography with a smooth initial model to estimate the P-velocity and the quality factor. Their velocity estimates are good, but quality factor estimates are less satisfying. Both cases are indecisive on the reason behind the poor attenuation estimations in the simultaneous case. An obvious and often-used method to overcome this is by separating the velocity updates from the attenuation updates (Hicks and Pratt, 2001; Rao and Wang, 2008). An alternative approach is to use a preconditioner to improve the final result (Hak and Mulder, 2008a,b).

The inconsistency of the results for visco-acoustic migration motivated us to investigate the performance of the preconditioned conjugate-gradient method. We limit our discussion to the constant density visco-acoustic wave equation in the frequency domain. The frequency-independent model parameter is complex-valued and is a cobination of the scattered wave velocity and scattered quality factor. We will see that the attenuation perturbations hardly contribute to the gradient and the shape of the gradient is mainly determined by the velocity perturbations. This has negative consequences for the update of the imaginary part of the model parameter. We will also see that different preconditioners lead to different estimates of the model parameters, despite convergence of the iterative algorithm.

In the next section, we briefly review the governing equations of constant density visco-acoustics and iterative migration. In the following section we describe our synthetic model. In Section 3.4, we explain the complications that arise when estimating velocity and attenuation perturbations by looking at the gradient that is used by migration for a simple point scatterer model. In section 3.5, we will perform the iterative migration of the model using different preconditioners, of which the results will be discussed in the final section.

\subsection{Governing equations}

\subsubsection{Constant density visco-acoustic wave equation}

The constant density visco-acoustic wave equation, or visco-scalar wave equation, in the frequency domain has a single, complex-valued parameter 
$m$. For a single source, with source function $s$, the wave equation reads

$$
\left[-\omega^{2} m(\omega, x)-\Delta\right] p(\omega, x)=s(\omega, x)
$$

where $p$ is the pressure wavefield, and $\Delta$ is the Laplace operator. For simplicity, we assume the quality factor is constant in the frequency range of interest. We also omit the correction for causality. The medium parameter $m$ then becomes independent of frequency. It equals the inverse of the squared effective wave velocity, given by

$$
m(x) \equiv \frac{1}{v^{2}(x)}=\frac{1}{c^{2}(x)}\left[1+\frac{\mathrm{i}}{Q(x)}\right]
$$

Here $v$ is the (complex-valued) effective velocity, $c$ is the wave velocity for a lossless medium, and $Q$ is the quality factor. Note that the real part of the parameter is related only to the velocity and the imaginary part incorporates the quality factor. The Born approximations splits Equation (3.1) into the pair

$$
\begin{aligned}
& {\left[-\omega^{2} m_{b}-\Delta\right] p_{b}(\omega, x)=s(\omega, x),} \\
& {\left[-\omega^{2} m_{b}-\Delta\right] \delta p(\omega, x)=\omega^{2} \delta m(\boldsymbol{x}) p_{b}(\omega, \boldsymbol{x}) .}
\end{aligned}
$$

Here, the model parameter $m$ is divided into a constant background model $m_{b}$ and perturbations $\delta m$ to this background model, such that $m(x)=$ $m_{b}+\delta m(\boldsymbol{x})$. In the case of a constant background model, all reflections are due to the perturbations. The background pressure wavefield $p_{b}$ is easily computed via the Green function.

\subsubsection{The Green function}

For a constant wave velocity $v_{b}=m_{b}^{-1 / 2}$, the visco-acoustic Green function $G$ at $x$ due to a source at $x_{\mathrm{s}}$ is given by

$$
G\left(\omega, x_{\mathrm{s}}, x\right)=\frac{\exp \left\{\mathrm{i} \omega R / v_{b}\right\}}{4 \pi R}
$$


with $R=\left|x_{\mathrm{s}}-x\right|$ and where $\mathrm{s}$ indexes source locations. Hence, for our Born approximation we have

$$
\left[-\omega^{2} m_{b}-\Delta\right] G\left(\omega, x_{\mathrm{s}}, x\right)=\delta\left(x-x_{\mathrm{s}}\right) .
$$

For a point source at $x_{\mathrm{s}}$ with frequency $\omega$ that is given by $s(\omega, x)=$ $w_{\mathrm{s}}(\omega) \delta\left(\boldsymbol{x}-\boldsymbol{x}_{\mathrm{s}}\right)$, the scattered pressure wavefield at a receiver $\boldsymbol{x}_{\mathrm{r}}$ then yields

$$
\delta p_{\mathrm{r}(\mathrm{s})}(\omega)=\omega^{2} w_{\mathrm{s}}(\omega) \int G\left(\omega, x, x_{\mathrm{r}}\right) \delta m(\boldsymbol{x}) G\left(\omega, x_{\mathrm{s}}, \boldsymbol{x}\right) \mathrm{d} \boldsymbol{x},
$$

where $\mathrm{r}(\mathrm{s})$ indexes receiver positions for this source. For a finite field, this can be expressed by a matrix-vector system for discreet subsurface positions $x_{k}$, where $\mathrm{k}$ indexes these subsurface positions. Suppose $\delta \mathbf{p}_{\mathrm{r}(\mathrm{s})}$ gives a vector with the pressure wavefield at the different positions, and $\delta \mathbf{m}$ the vector of medium parameters per location. Then, the system

$$
\delta \mathbf{p}_{\mathrm{r}(\mathrm{s})}(\omega)=\mathbf{F}_{\mathrm{r}(\mathrm{s})} \delta \mathbf{m}
$$

is the discretised representation of Equation (3.4).

\subsubsection{Least-squares minimisation}

Define the error for a source at $x_{\mathrm{s}}$ as the difference between simulated scattered data $\delta \mathbf{p}_{\mathrm{r}(\mathrm{s})}$ at receiver locations $\boldsymbol{x}_{\mathrm{r}(\mathrm{s})}$ and observed data $\mathbf{p}_{\mathrm{r}(\mathrm{s})}^{\text {obs }}$. Then, the least-squares cost functional $\mathcal{J}$ for all receivers, sources, and frequencies, is given by

$$
\mathcal{J}=\sum_{\omega, s, \mathrm{r}(\mathrm{s})}\left\|\delta \mathbf{p}_{\mathrm{r}(\mathrm{s})}(\omega)-\mathbf{p}_{\mathrm{r}(\mathrm{s})}^{o b s}(\omega)\right\|^{2} .
$$

This cost functional is expressed as a function of complex-valued arguments. Note that it differs from the more common definition of a leastsquares cost functional that contains a factor $\frac{1}{2}$. Instead of using the real and imaginary part of the complex model parameter vector $\delta \mathbf{m}$, which is one way of dealing with differentiation of a function that is not analytic, we use $\delta \mathbf{m}$ and its complex conjugate $\delta \mathbf{m}^{*}$ as two distinct parameter vectors. Van den Bos (1994), among others, gave a short description of this 
approach, including the first and second derivatives of the least-squares cost functional with respect to the model parameters. These derivatives can then be used by the conjugate-gradient method to compute the model parameters that explain the data in the 'best' way.

From a Taylor series expansion, it follows that the minimum of equation (3.6) with respect to the model parameters solves the system

$$
\mathbf{H} \delta \mathbf{m}=-\mathbf{g},
$$

where $\mathbf{H}$ is a Hessian matrix, or second derivative of the cost functional with respect to the perturbation parameters, and $g(x)$ is the gradient, or first derivative of the cost functional with respect to the perturbation. The gradient and Hessian are given by

$$
\mathbf{g}=\sum_{\omega, \mathrm{s}, \mathrm{r}(\mathrm{s})} \mathbf{F}_{\mathrm{r}(\mathrm{s})}^{\mathrm{H}}\left[\delta \mathbf{p}_{\mathrm{r}(\mathrm{s})}(\omega)-\mathbf{p}_{\mathrm{r}(\mathrm{s})}^{o b s}(\omega)\right] .
$$

and

$$
\mathbf{H}=\sum_{\omega, \mathrm{s}, \mathrm{r}(\mathrm{s})} \mathbf{F}_{\mathrm{r}(\mathrm{s})}^{\mathrm{H}} \mathbf{F}_{\mathrm{r}(\mathrm{s})} .
$$

Here, the superscript $(\cdot)^{\mathrm{H}}$ denotes the complex conjugate transpose. Note that both the gradient and the Hessian are complex-valued functions.

\subsection{Synthetic example}

Our synthetic example consists of a single point scatterer in an otherwise homogeneous background medium. Analytic expressions for the observed data, the gradient, and the Hessian are readily available using equations (3.5) - (3.9). At position $x_{q}$, the model is perturbed in both the real and imaginary part. We will refer to the real part of the parameter as the first parameter and to the imaginary part as the second parameter, denoted as $\delta m^{(1)}$ and $\delta m^{(2)}$, respectively. The first parameter is related to perturbations of the wave velocity, the second parameter also incorporates perturbations of the quality factor.

The synthetic model has a constant background model with a velocity $c_{b}$ of $2000 \mathrm{~m} / \mathrm{s}$ and a quality factor $Q_{b}$ of 50 . At the point scatterer $x_{\mathrm{q}}$, 

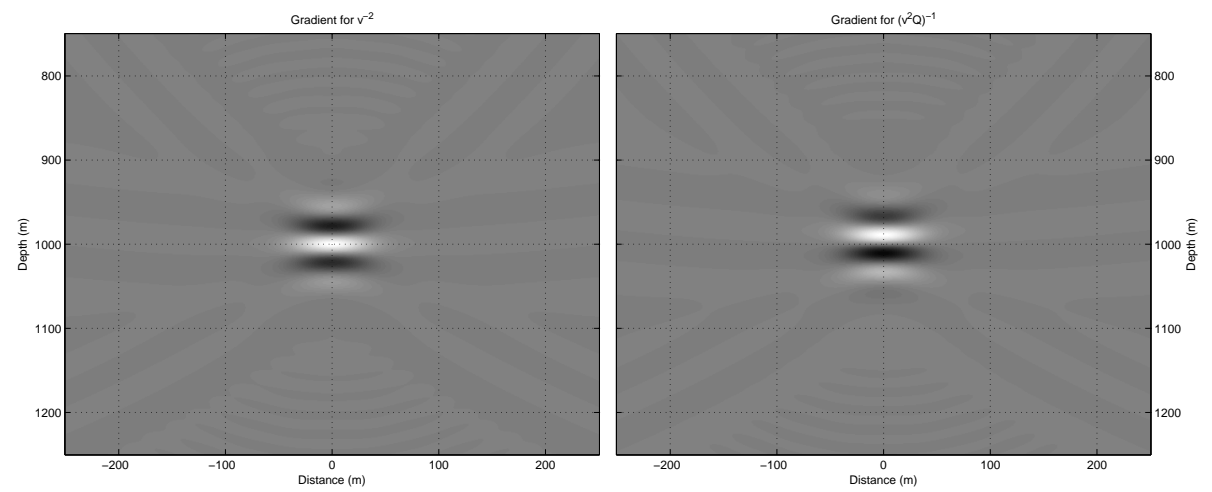

Figure 3.1: The real (left panel) and imaginary (right panel) part of the gradient for the model described in this article. The grey scale is set to the absolute maximum of each panel separately, with white being positive and black being negative.

the true model has a velocity $c$ of $2500 \mathrm{~m} / \mathrm{s}$ and a quality factor $Q$ of 80 . This means that our linear, perturbed parameters at the scatterer are given by $\delta m^{(1)}\left(x_{\mathrm{q}}\right)=-9 \cdot 10^{-8}(\mathrm{~s} / \mathrm{m})^{2}$ and $\delta m^{(2)}\left(x_{\mathrm{q}}\right)=-3 \cdot 10^{-9}(\mathrm{~s} / \mathrm{m})^{2}$ and zero elsewhere. Receivers are positioned on a $1800 \mathrm{~m}$ line every $25 \mathrm{~m}$ and sources are placed mid-way between each pair of successive receivers. The frequencies range from $4 \mathrm{~Hz}$ to $44 \mathrm{~Hz}$ and we use a Ricker wavelet with a peak frequency of $20 \mathrm{~Hz}$. The scatterer is located at a depth of $1000 \mathrm{~m}$ beneath the central receiver.

Figure 3.1 shows the gradient in the target area for the two-dimensional plane containing the scatterer and source-receiver line. For the image, a finer grid was used than for the iterative scheme later on.

\subsection{Gradient}

The gradient is the first derivative of the cost functional with respect to the model parameters. For a gradient-based method, it gives the direction of the update, but in general with the wrong step length. For migration, the gradient gives the scatterer location, but in general has a wrong amplitude. The scatterer locations and amplitudes can be improved by using an iterative method, e.g., a conjugate-gradient method. The method benefits 
from proper weighting of the gradient by preconditioning, where the optimal preconditioner is the pseudo-inverse of the Hessian that is usually too costly to compute.

Ideally, the gradient has a true-amplitude spike at scatterer locations only and should be zero elsewhere. In that case, the estimated model will be updated only at the true scatterer location with the correct value. Ideally, the gradient with respect to a perturbed parameter has this spike for the perturbed parameter only and it has no crosstalk with other parameters, meaning the gradient updates only the parameter responsible for the perturbation. For several reasons, e.g., acquisition geometry or band-limited data, the actual gradient differs from this ideal case and has artifacts that could harm the final result.

The question is now why iterative migration for the constant density visco-acoustics is difficult when we want to simultaneously retrieve the parameter. For our synthetic model, a vertical cross-section of the gradient through the scatterer is shown in Figure 3.2. Because our equations are linear in the perturbation parameter, the gradient with respect to the perturbation parameter $\delta m(x)$ is the sum of the gradient with respect to the first parameter $\delta m^{(1)}(x)$ and i times the gradient with respect to the second parameter $\delta m^{(2)}(\boldsymbol{x})$.

The gradient with respect to the first parameter (red) shows a symmetric behaviour around the scatterer in the left panel and an anti-symmetric behaviour around the scatterer in the right panel. This gradient for the first parameter peaks at the desired location (left panel), but also shows a contribution of equal amplitude - an artifact - to the second parameter (right panel). The reverse is true for the gradient with respect to the second parameter (blue) and its contribution to the first parameter, although this can hardly be seen in the figure. However, the amplitude of the gradient with respect to the second parameter is much smaller than the one with respect to the first. Its sum, that is, the full gradient (dashed, black) is practically on top of the gradient for the first parameter and the influence of gradient with respect to the second parameter is negligible.

Figure 3.3 displays a horizontal line of the gradient, passing through the scatterer, where the amplitude scale of the right panel is boosted 20 times. The behaviour is symmetric in both panels with a peak at the correct location. Also, in the right panel the amplitude of the gradient for the second parameter is a few times larger than the artifact of the first parameter gradient. In the horizontal direction, the gradient appears to be 

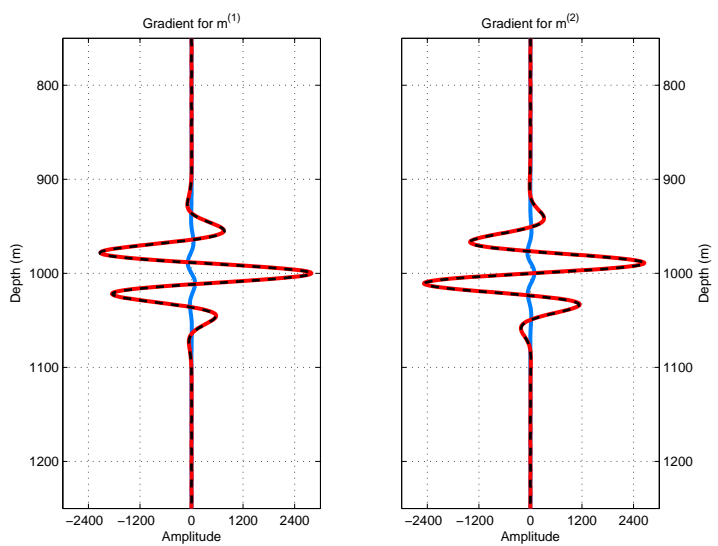

Figure 3.2: Real (left panel) and imaginary (right panel) part of the vertical crosssection through the point scatterer of the gradient for a medium perturbated in both the real and imaginary part (dashed, black), for a medium with real-valued perturbation only (red) and with an imaginary-valued perturbation only (blue). The amplitude scale is the same in both panels. Note the red line and the dashed black line are on top of each other.
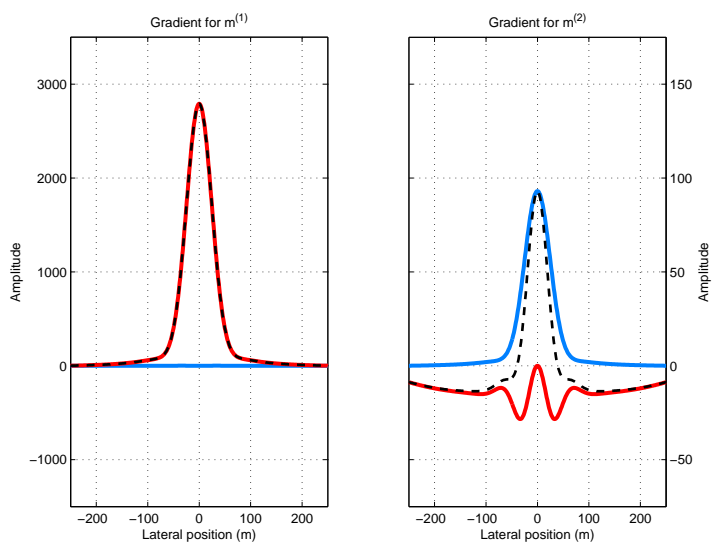

Figure 3.3: As figure 3.2, but now for a horizontal cross-section. Note that the right panel is magnified 20 times in comparison with the left panel. 
showing the desired characteristics and we conclude that the undesired behaviour occurs mainly in the depth direction.

Figure 3.1 confirms that the behaviour we see in the one-dimensional cross-sections (i.e., that the image of the second parameter is exceeded by the artifact of the first parameter) is mainly present in vertical direction. To be more precise, we observe that this artifact shows up in lines from the source-receiver array through the scatterer. In our setting, with a sourcereceiver array that lies symmetric around the much deeper scatterer, this means these lines are almost vertical.

\subsection{Preconditioner results}

The inverse problem of determining the perturbation parameters from the data is linear. Gradient-based minimisation of the least-squares functional with the conjugate gradient method is a common choice. A suitable preconditioner should accelerate the convergence of this method. The main problem during inversion is the disentanglement of the first and second parameter. The artifacts of the gradient associated with the first parameter, observed in the right panel of Figure 3.1 and 3.2, tends to hide the contribution of the second parameter. Since this behaviour is influenced by the acquisition geometry and finite bandwidth of the data, one could investigate their effect on the quality of the inversion result. This topic is not addressed here. Instead, we consider different preconditioners and investigate its performance in a conjugate-gradient update.

For a conjugate-gradient method, the condition number or the spectrum of the eigenvalues of the coefficient matrix are an important factor for the rate of convergence rate (Shewchuk, 1994). If the condition number is small, the amount of iterations for the method to find the solution is at most the number of unknowns, but if the matrix is nearly singular, the condition number is high and convergence becomes slow. Also, the result of a conjugate gradient method for a nearly singular system is unreliable. A preconditioner may be used to improve the condition number of the coefficient matrix.

We consider six preconditioners to do such, which we define in pairs and denote with roman I, II, and III. Group I has equal weights for the first and second parameter, whereas groups II and III have different weights for the first parameter. Group II has the weights $c_{b}^{2}$ and $c_{b}^{2} Q_{b}$ for the first and 

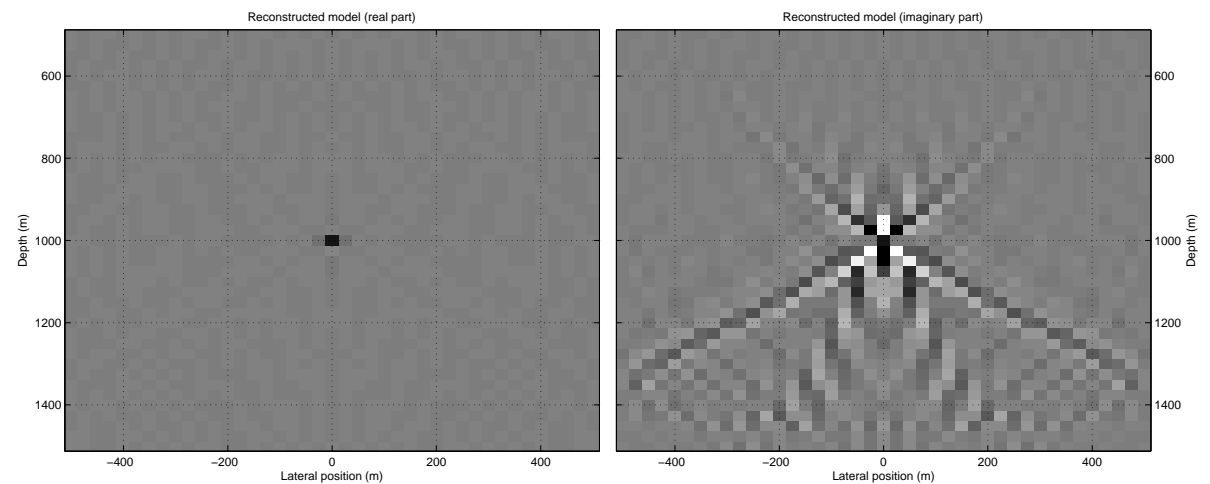

Figure 3.4: Estimates of the first (left panel) and second (right panel) model parameter after 2000 iterations using preconditioner $\mathrm{Ib}$.

second parameter, respectively, and group III has the weights 1 for the first and $c_{b}^{2} Q_{b}$ for the second parameter. The pairs are different with respect to the diagonal values, indicated by letters ' $a$ ' and ' $b$ ', where ' $b$ ' indicates we multiplied the preconditioner matrix with the diagonal of the Hessian matrix.

For our tests, we restrict ourselves to the vertical plane containing the source-receiver array. For the synthetic model, we graphed the gradient for the first and second parameter in Figure 3.1 with a $1.25 \mathrm{~m}$ grid spacing, although our tests are spaced on a $25 \mathrm{~m} \mathrm{grid}^{1}$. We investigated these preconditioners to retrieve both parameters of the original perturbation model, where the background model is known and fixed. The scheme starts with an initial estimate where both parameters equal to zero. For each preconditioner labelled with ' $b$ ', the retrieved model after 2000 iterations is given in Figures 3.4-3.6. The corresponding relative residual is given in Figure 3.7 to show the convergence of the method.

The methods are compared by measuring two errors in the final answer, where we use the Euclidean norm. First, the relative error $\varepsilon$ defined as the norm of the difference between the true model and the estimate model,

\footnotetext{
${ }^{1}$ The spacing was limited by the computationally intensive Hessian that already has $3 \cdot 10^{6}$ elements on the $25 \mathrm{~m}$ spaced grid.
} 

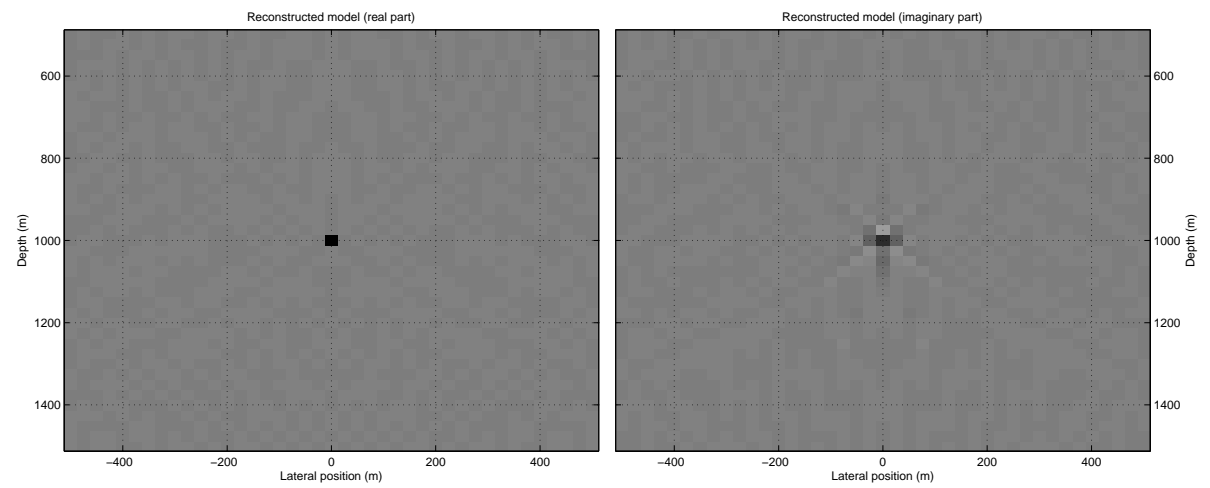

Figure 3.5: As Figure 3.4 for preconditioner $\Pi b$.
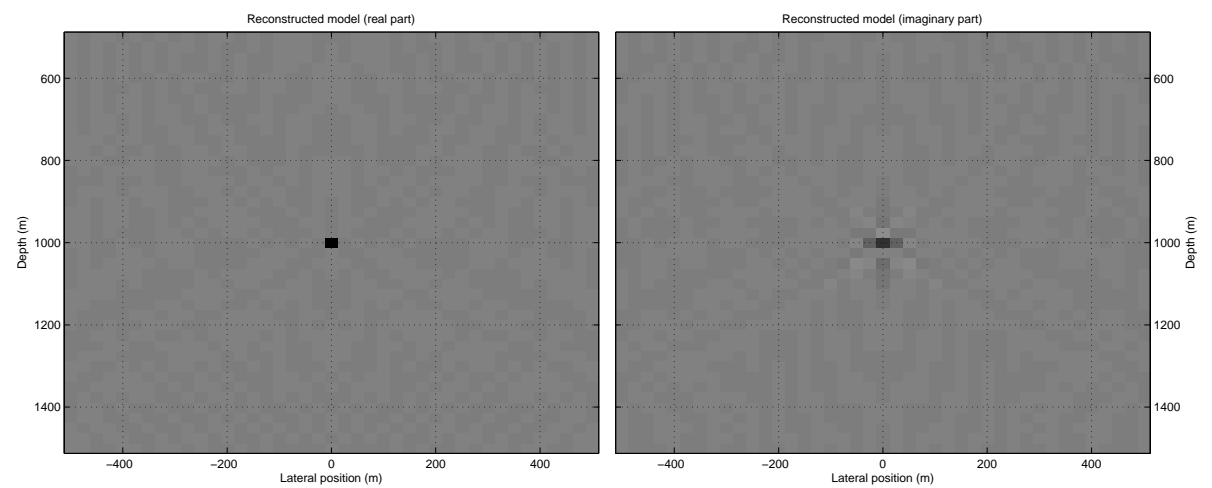

Figure 3.6: As Figure 3.4 for preconditioner $\mathrm{III} b$.

and second, $\varepsilon^{\mathrm{sc}}$, the relative error at the scatterer location:

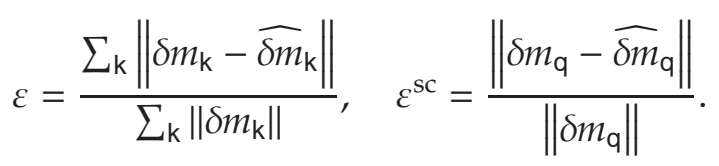

where $\delta m_{\mathrm{k}}=\delta m\left(\boldsymbol{x}_{\mathrm{k}}\right)$ is the true model in the point $x_{\mathrm{k}}, \widehat{\delta m}_{\mathrm{k}}$ is the estimated model in the same point. Index $q$ indicates the scatterer point.

We performed updates for a fixed number of iterations, up to $10^{6}$ in 


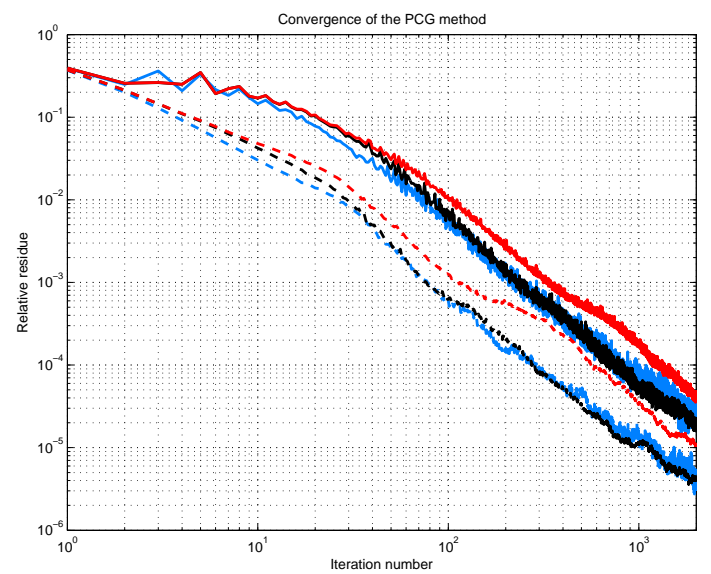

Figure 3.7: Convergence of all preconditioners after 2000 iterations, plotted on a double logarithmic scale. Each cross represents a separate run. Group I is blue, group II is black, group III is red, types ' $a$ ' are solid lines, and types ' $b$ ' are dashed.
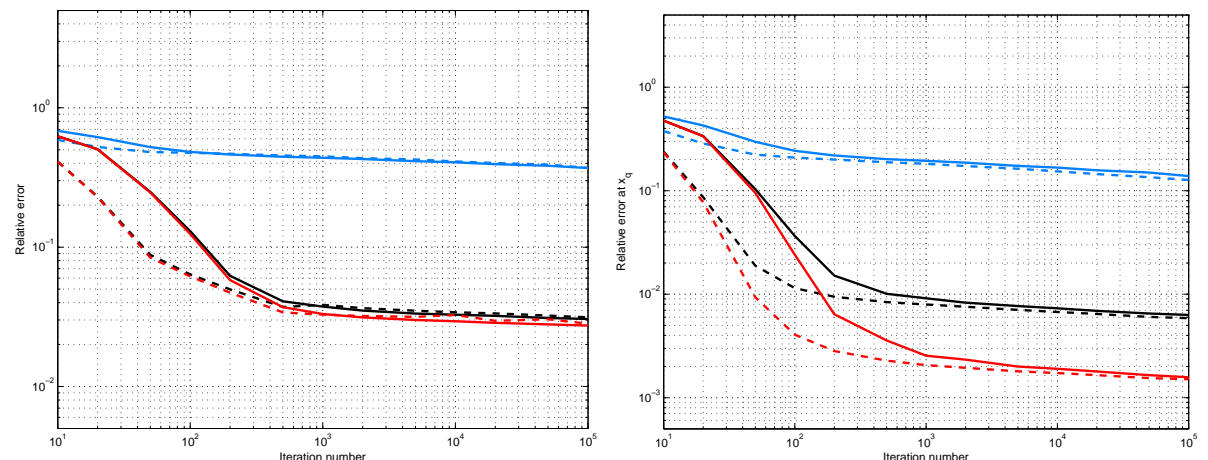

Figure 3.8: The relative error (left panel) and the relative error at the scatterer (right panel) in the final estimate versus the total number of iterations for different preconditioners, plotted on a double logarithmic scale. Group I is blue, group II is black, group III is red, types ' $a$ ' are solid lines, and types ' $b$ ' are dashed. 
some cases, to show the trend of these errors. Results for the tests are shown in Figure 3.8.

\subsection{Summary and conclusion}

We performed iterative migration for the constant density visco-acoustic wave equation, where reflections are due to changes in a complex-valued velocity. We used a conjugate-gradient method for updating and tested the performance of several preconditioners.

We have seen that the amplitude of the gradient for a model that is perturbed only for the second model parameter is much smaller in comparison with that of only the first model parameter. Consequentially, when the model is perturbed simultaneously, the gradient will be dominated by the gradient with respect to the first model parameter. The gradient and with it the direction of the update, is fully driven by the gradient with respect to the first parameter.

In Figure 3.7 we see the method is converging, but from Figures 3.4-3.6 we can observe it converges to at least two different models. Theoretically in case of a well-posed problem, 2000 iterations should be enough to accurately find all unknowns, since this is the approximate amount of unknowns. Figure 3.8 confirms that different models are found for different preconditioners indicating the problem is ill-posed. Basically, we can select a solution by means of the preconditioner.

We conclude it is not possible to simultaneously find a reliable velocity and attenuation estimate in this setting. There is a fundamental problem in getting reliable true-amplitude migration results for the constant density visco-acoustic wave equation. This problem will be addressed in the subsequent chapters. 


\title{
Chapter 4
}

\section{An ambiguity in attenuation scattering imaging}

\begin{abstract}
Migration constructs a subsurface image by mapping band-limited seismic data to reflectors in the Earth, given a background velocity model that describes the kinematics of the seismic waves. Classically, the reflectors correspond to impedance perturbations on length scales of the order of the seismic wavelength. The Born approximation of the visco-acoustic wave equation enables the computation of synthetic data for such a model. Migration then amounts to solving the linear inverse problem for perturbations in density, velocity, and attenuation.

Here, the problem is simplified by assuming the density to be constant, leaving only velocity and attenuation perturbations. In the frequency domain, a single complex-valued model parameter that depends on subsurface position describes both. Its real part is related to the classic reflectivity, its imaginary part also involves attenuation variations. Attenuation scattering is usually ignored but, when included in the migration, might provide information about, for instance, the presence of fluids. We found, however, that it is very difficult to solve simultaneously for both velocity and attenuation perturbations. The problem already occurs when computing synthetic data in the Born approximation for a given scattering model:
\end{abstract}


after applying a weighted Hilbert transform in the depth coordinate to a given scattering model, we obtained almost the same synthetic data if the scatterers had small dip and were located at not-too-shallow depths. This implies that it will be nearly impossible to simultaneously determine the real and imaginary part of the scattering parameters by linearized inversion without imposing additional constraints.

\subsection{Introduction}

The oil and gas industry acquires seismic data to obtain an image of the subsurface that may reveal hydrocarbon bearing formations. Because a 3-D full visco-elastic inversion is computationally still out of reach, various approximations of the wave equation are employed, often based on ray tracing or one-way wave equations. These are often sufficiently accurate to obtain a structural image. A precise characterization of the subsurface that allows for volumetric estimates of the amounts of hydrocarbons in place requires a more accurate description. Visco-acoustic or visco-elastic full waveform inversion is computationally tractable in a 2-D approximation. However, the presence of local minima in the least-squares misfit functional makes the solution of the inverse problem difficult. One cause for this problem is the absence of low frequencies, below $8-10 \mathrm{~Hz}$, in the seismic data.

The inverse problem becomes considerably simpler when linearized. Classic methods for velocity analysis provide a background velocity model. An operation called migration maps the band-limited seismic data to reflectors in the subsurface. Mathematically, this method amounts to a single iteration of a gradient-based minimization of the least-squares misfit functional between observed and modelled data, using the Born approximation of the wave equation. Because this approximation assumes single scattering, the direct arrival and multiple reflections should be removed from the data before migration.

Examples (Østmo et al., 2002; Mulder and Plessix, 2004) show that with proper weighting or preconditioning (Plessix and Mulder, 2004), one or a few iterations with the conjugate-gradient method suffice to obtain a solution to the inverse problem when using the Born approximation of the constant-density visco-acoustic wave equation. The method works in the frequency-domain and reconstructs scatterers that represent perturba- 
tions of the background velocity model. In the frequency-domain, these are represented by complex numbers. The real part is almost entirely related to impedance perturbations, usually caused by abrupt changes in rock properties. If we include the imaginary part, we can formally obtain both velocity and attenuation perturbations. The latter, however, appeared to have no relation to physically realistic values in our numerical experiments.

To better understand why it may be difficult to reconstruct the imaginary part of the scattering perturbations, we consider the simple case of a 1-D model consisting of horizontally layered scatterers in a homogeneous background for the constant-density visco-acoustic equation in Section 4.2. The scatterers correspond to perturbations both in velocity and attenuation. In Section 4.3, we derive the scattered wave field in the high-frequency case by means of the method of stationary phase. It turns out that there is an ambiguity that already occurs at the forwardmodelling level: if we apply a weighted Hilbert transform in the depth coordinate to the scatterers, we obtain almost the same reflection data as for the original scatterers. As a consequence, least-squares fitting of observed data will lead to an inverse problem that is close to singular. We show that the 'true-amplitude' migration of the data, which amounts to the first iteration of a preconditioned conjugate-gradient minimization of the least-squares error, produces a reconstruction of the perturbations that is the average of the original and the transformed scattering model and has a minimum-norm property.

In Section 4.4, we address the question to what extent these conclusions carry over to a complex velocity model and dipping reflectors. We perform 2-D finite-difference simulations with a frequency-domain code that solves the system of equations that represents the Born approximation of the constant-density visco-acoustic wave equation.

We discuss the results in Section 4.5 and summarize the main conclusion in Section 4.6.

\subsection{Governing equations}

Migration maps seismic data recorded at the Earth's surface into an image of the subsurface. The implicit assumption is that the data only contain primaries and that surface or interbed multiples are absent or negligible. 
We can generate such data using the Born approximation of the wave equation. In this approximation, the subsurface model is split into a part that does not produces significant reflections in the seismic frequency band and perturbations that generate the reflection data. For the acoustic wave equation, reflections can be avoided by defining the density in such a way that the impedance is constant. For constant-density acoustics, we use a smooth background velocity. The perturbations are then obtained as the difference between the original rough velocity model and its smoothed version that serves as the background model.

In the frequency domain, the constant-density visco-acoustic wave equation is

$$
-\frac{\omega^{2}}{v^{2}} \hat{p}-\Delta \hat{p}=f .
$$

Here $\hat{p}(\omega, x)$ the pressure, $\omega=2 \pi f$ with $f$ the frequency, $f(\omega, x)$ is a source term, and the complex velocity $v(\omega, x)$ is given by ( $c f$. Aki and Richards, 2002, eq. 5.94)

$$
\frac{1}{v}=\frac{1}{c}\left[1-\frac{1}{\pi Q} \log \left(f / f_{r}\right)+\frac{\mathrm{i}}{2 Q}\right],
$$

with real-valued velocity $c(x)$ and quality factor $Q(x)$. The latter is usually much larger than 1 . Causality requires the logarithmic term with reference frequency $f_{r}$.

By linearizing eq. (4.1) with respect to the model, we obtain the Born approximation

$$
-\omega^{2} m_{0} \hat{p}_{0}-\Delta \hat{p}_{0}=f, \quad-\omega^{2} m_{0} \hat{p}_{1}-\Delta \hat{p}_{1}=\omega^{2} m_{1} \hat{p}_{0},
$$

with model parameters $m=v^{-2}=m_{0}+m_{1}$, where $m_{0}(\omega, x)=v_{0}^{-2}$ represents a smooth background velocity model $v_{0}$ that should not produce significant scattering in the seismic frequency band and $m_{1}(\omega, x)=v^{-2}-v_{0}^{-2}$ a perturbation term that is responsible for the scattering of incoming waves. For simplicity, we will assume that $m_{1}(\omega, \boldsymbol{x})=m_{1}(\boldsymbol{x})$, independent of $\omega$.

For the Fourier transform in time, we adopt the convention

$$
p(x, t)=(2 \pi)^{-1} \int_{-\infty}^{\infty} \hat{p}(\omega, x) e^{-\mathrm{i} \omega t} \mathrm{~d} \omega .
$$


We will denote the Hilbert transform of a function $g(z)$, depending on $z$, by $\mathcal{H}_{z}[g]$. This amounts to convolution with $(\pi z)^{-1}$ in the depth domain.

\subsection{3-D homogeneous background with 1-D scatte- ring model}

\subsubsection{Scattered wavefield}

We make the following simplifying assumptions. The background velocity model is defined by the constants $c_{0}$ and $Q_{0}$, whereas the perturbation $m_{1}(z)$ only depends on depth. The source is a delta function located at $x_{s}=-h<0, y_{s}=0, z_{s}=0$, and we consider a single receiver at $x_{r}=+h$, $y_{r}=0, z_{r}=0$. We assume that $\omega$ is finite but large enough to justify the application of the method of stationary phase.

The background pressure is given by the 3-D Green's function

$$
\hat{p}_{0}(\omega, x)=\frac{e^{i k_{0} r_{s}}}{4 \pi r_{s}}, \quad k_{0}=\frac{\omega}{v_{0}}, \quad r_{s}=\sqrt{(x+h)^{2}+y^{2}+z^{2}} .
$$

Note that $k_{0}$ is complex because $v_{0}$ is. The scattered field at the receiver is

$$
\hat{p}_{1}(\omega, h)=\int_{-\infty}^{\infty} \int_{-\infty}^{\infty} \int_{0}^{\infty} \frac{\omega^{2} e^{\mathrm{i} k_{0}\left(r_{s}+r_{r}\right)}}{16 \pi^{2} r_{s} r_{r}} m_{1}(z) \mathrm{d} z \mathrm{~d} y \mathrm{~d} x,
$$

with $r_{r}=\sqrt{(x-h)^{2}+y^{2}+z^{2}}$. We assume that scattering only occurs beyond a depth $z_{m}>0$, so $m_{1}(z)=0$ for $z \leq z_{m}$.

Integration over $x$ and $y$ of eq. (4.4) with the method of stationary phase leads to

$$
\hat{p}_{1}(\omega, h) \simeq \frac{\mathrm{i} \omega c_{0}}{16 \pi} \int_{0}^{\infty} f_{1}(z) \mu(z) \mathrm{d} z,
$$

with

$$
f_{1}(z)=e^{2 \mathrm{i} k_{0} \sqrt{z^{2}+h^{2}}}, \quad \mu(z)=\frac{m_{1}(z)}{z} .
$$




\subsubsection{Ambiguity}

While inspecting migration images obtained with a 2-D constant-density visco-acoustic finite-difference code, we noted that the real and imaginary parts resembled each other's Hilbert transform. This suggested that demigration, which amounts to forward modelling of the scattering model obtained by migration, would yield data that should closely resemble the original data. This indeed turned out to be the case and led to the conclusion that a given scattering model and its Hilbert transform, with suitable weighting and scaling, would produce the same data. For the simplified model problem considered in this section, this means that $\int_{-\infty}^{\infty} f_{1} \mu \mathrm{d} z$ should be the same as $-\mathrm{i} \int_{-\infty}^{\infty} f_{1} \mathcal{H}_{z}[\mu] \mathrm{d} z$. Because

$$
\int_{-\infty}^{\infty} f_{1} \mathcal{H}_{z}[\mu] \mathrm{d} z=-\int_{-\infty}^{\infty} \mu \mathcal{H}_{z}\left[f_{1}\right] \mathrm{d} z
$$

we can proceed without $\mu(z)$ and concentrate on $\mathcal{H}_{z}\left[f_{1}\right]$. If we could prove that i $\mathcal{H}_{z}\left[f_{1}\right]=f_{1}$, then we would have demonstrated that $\mu$ and $-\mathrm{i} \mathcal{H}_{z}[\mu]$ would lead to the same data for arbitrary $\mu(z)$, so for any scattering model. It will turn out that i $\mathcal{H}_{z}\left[f_{1}\right]$ and $f_{1}$ are different, but that the difference may be small.

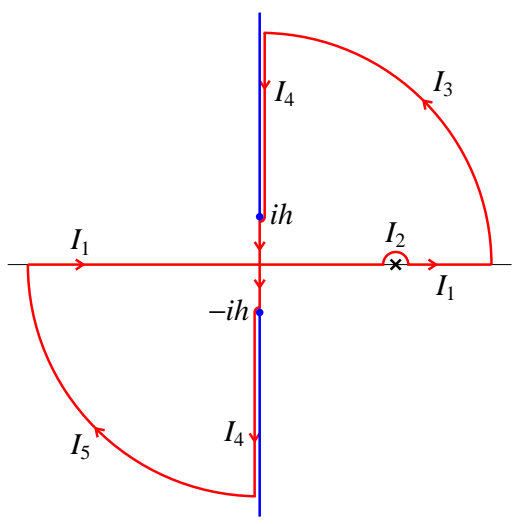

Figure 4.1: Two closed contours are glued together to evaluate $\mathcal{H}_{z}\left[f_{1}\right]$. The blue lines mark the branch-cuts of $\sqrt{h^{2}+\zeta^{2}}$. The cross indicates the pole. 
To evaluate the integral $I_{1}=\mathcal{H}_{z}\left[f_{1}\right]$ we consider the closed contour $C$ sketched in Fig. 4.1, which can be interpreted as two closed contours glued together, one the first quadrant of the complex plane and one in the third. The integral

$$
I(z)=\frac{1}{\pi} \int_{C} \frac{f_{1}(\zeta)}{z-\zeta} \mathrm{d} \zeta=0 .
$$

Now $I=I_{1}+I_{2}+I_{3}+I_{4}+I_{5}$, with $I_{1}=\mathcal{H}_{z}\left[f_{1}\right] I_{2}$ the contribution at the pole $\zeta=z, I_{3}$ the result for the arc in the first quadrant when its radius goes to infinity, $I_{4}$ the integral over the imaginary axis downward, where we have to stay on the proper side of the branch-cut of $\sqrt{h^{2}+\zeta^{2}}$, and $I_{5}$ the result for the arc in the third quadrant. The residual theorem yields $I_{2}=\mathrm{i} f_{1}(z)$ at the pole if $z>0$. For $z<0$, we have to traverse the pole, which now lies on the negative real axis, from below, resulting in $I_{2}=-\mathrm{i} f_{1}(z)$. Therefore, $I_{2}(z)=\mathrm{i} f_{1}(z) \operatorname{sign}(z)$. The arcs provide $I_{3}=0$ and $I_{5}=0$. The integral over the imaginary axis leads to

$$
\left.I_{4}=-\frac{1}{\pi} \int_{-\infty}^{\infty} \frac{e^{2 \mathrm{i} k_{0}} \sqrt{h^{2}-\tilde{\eta}^{2}}}{z-\mathrm{i} \tilde{\eta}} \mathrm{d}(\mathrm{i} \tilde{\eta})=-\mathrm{i}\left[f_{2}(z)+f_{3}(z)\right)\right]
$$

with

$$
f_{2}(z)=\frac{2 \tilde{z}}{\pi} \int_{0}^{1} \frac{e^{\mathrm{i} \alpha} \sqrt{1-\eta^{2}}}{\tilde{z}^{2}+\eta^{2}} \mathrm{~d} \eta, \quad f_{3}(z)=\frac{2 \tilde{z}}{\pi} \int_{1}^{\infty} \frac{e^{-\alpha} \sqrt{\eta^{2}-1}}{\tilde{z}^{2}+\eta^{2}} \mathrm{~d} \eta .
$$

Here $\tilde{z}=z / h$ and $\alpha=2 k_{0} h$ with $\alpha_{r}=\operatorname{Re} \alpha>0$ and $\alpha_{i}=\operatorname{Im} \alpha>0$. As a result,

$$
f_{4}(z)=i \operatorname{sign}(z) \mathcal{H}_{z}\left[f_{1}\right]=f_{1}+\left(f_{2}+f_{3}\right) \operatorname{sign}(z) .
$$

We have now found that $\mathrm{i} \mathcal{H}_{z}\left[f_{1}\right]$ and $f_{1}$ are different. Apart from the extra factor, $\operatorname{sign}(z)$, which is just a technicality if $\mu(z)=0$ for $z \leq 0$, there is a remainder $\left(f_{2}+f_{3}\right) \operatorname{sign}(z)$. If we could show that both $\left|f_{2}\right|$ and $\left|f_{3}\right|$ are significantly smaller than $\left|f_{1}\right|$, we would have the relation $i \operatorname{sign}(z) \mathcal{H}_{z}\left[f_{1}\right] \simeq f_{1}$. Then, the recorded pressure is proportional to

$$
\int_{-\infty}^{\infty} f_{1}(z) \mu(z) \mathrm{d} z \simeq-\mathrm{i} \int_{-\infty}^{\infty} f_{1}(z) \mathcal{H}_{z}[\mu(z) \operatorname{sign}(z)] \mathrm{d} z,
$$


which implies that almost the same data will be observed for $m_{1}(z)$ and $\tilde{m}_{1}(z)=-\mathrm{i}|z| \mathcal{H}_{z}\left[m_{1}(z) / z\right]$

Before considering the remainder $\left(f_{2}+f_{3}\right) \operatorname{sign}(z)$, we will use eq. (4.6) to refine the definition of the depth-weighted, scaled Hilbert transform. We assumed earlier that $m_{1}(z)=0$ for negative $z$. The transformed model $\tilde{m}_{1}(z)$, however, may have non-zero values for negative $z$. Because $f_{1}(z)$ is symmetric in $z$, we can take $\tilde{m}_{1}(z)$ for $z<0$, mirror it with respect to $z=0$, and add it to the transformed model at positive $z$. This can be summarized by defining the depth-weighted, scaled Hilbert transform of a function $g(z)$ as

$$
\mathcal{M}_{z}[g](z)=\tilde{g}(z)+\tilde{g}(-z), \quad \tilde{g}(z)=-\mathrm{i}|z| \mathcal{H}_{z}[g / z],
$$

where $z \geq 0$ and we assume that $g(z)=0$ for $z \leq 0$.

It still remains to be shown under which conditions the term in eq. (4.6) with $f_{2}$ and $f_{3}$ is small relative to $f_{1}$. We list some bounds that may help to evaluate for which parameters of the background model and for which depths, given by $\tilde{z}=z / h, f_{2}$ and $f_{3}$ are much smaller than $f_{1}$. We have

$$
\left|f_{1}\right|=e^{-\alpha_{i} \sqrt{1+\tilde{z}^{2}}}
$$

and, assuming $\tilde{z} \geq 0$,

$$
\left|f_{2}\right| \leq \frac{2 \tilde{z}}{\pi} \int_{0}^{1} \frac{1}{\tilde{z}^{2}+\eta^{2}} \mathrm{~d} \eta=\frac{2}{\pi} \arctan (1 / \tilde{z}),
$$

whereas

$$
\left|f_{3}\right| \leq \frac{2 \tilde{z}}{\pi} \int_{1}^{\infty} \frac{e^{-\alpha_{r}(\eta-1)}}{1+\eta^{2}} \mathrm{~d} \eta=\frac{2}{\pi \alpha_{r}} \frac{\tilde{z}}{1+\tilde{z}^{2}} .
$$

The bounds for $\left|f_{2}\right|$ and $\left|f_{3}\right|$ are not very sharp.

To understand the bound on $f_{1}$, recall that $\alpha=2 k_{0} h=\alpha_{r}+\mathrm{i} \alpha_{i}$. If the damping of the background model is small, then $\alpha_{i} \ll \alpha_{r}$ and $\operatorname{Re} k_{0} \simeq \omega / c_{0}$. The bound for $f_{1}$ merely states that the data amplitudes for larger depths will decrease due to damping. Note that the amplitude decay due to the geometrical spreading is contained in the factor $1 / z$ that was absorbed in 

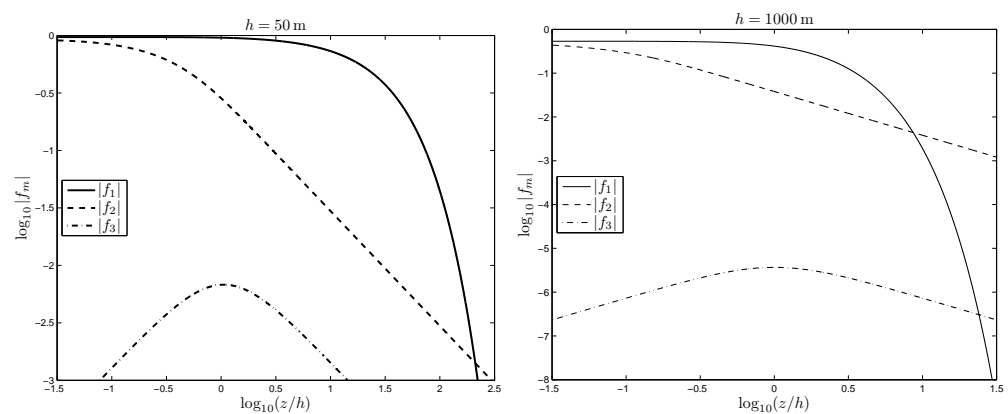

Figure 4.2: Moduli of $f_{1}, f_{2}, f_{3}$ at half-offsets of 50 and $1000 \mathrm{~m}$, showing that $f_{1}$ dominates $f_{2}$ and $f_{3}$ over a substantial range of depths. Note the difference in range for the vertical axis between the two cases.
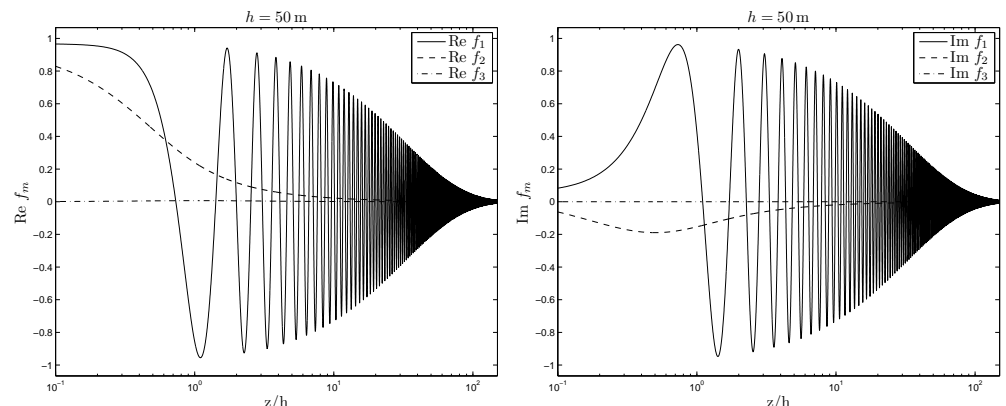

Figure 4.3: Real (left-hand panel) and imaginary (right-hand panel) part of $f_{1}, f_{2}$, $f_{3}$ at a half-offset of $50 \mathrm{~m}$, showing that $f_{1}$ dominates $f_{2}$ and $f_{3}$ over a substantial range of depths but is also far more oscillatory. Note the logarithmic scale for $z / h$.
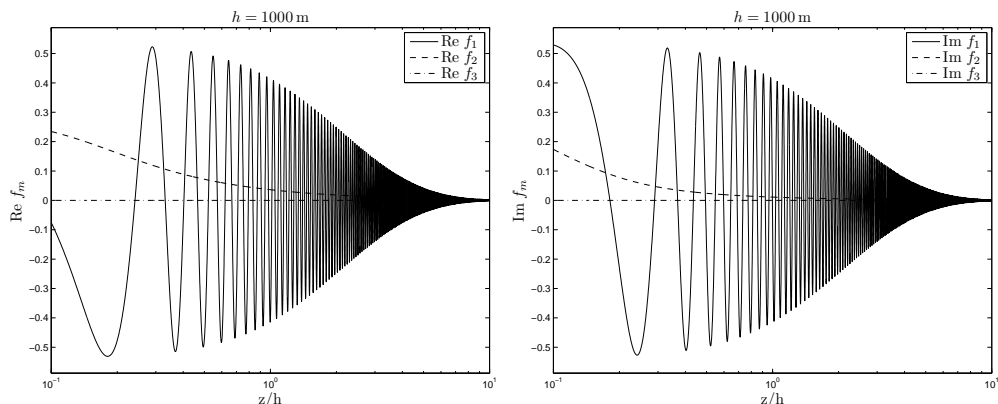

Figure 4.4: As Fig. 4.3, but for a half-offset of $1000 \mathrm{~m}$. 
the definition of $\mu$. If $\left|f_{1}\right|$ becomes significantly smaller than 1 , then the data will not be useful in practice when noise is present.

The bound on $f_{3}$ contains $\alpha_{r}$, which generally is much larger than $1-$ otherwise the method of stationary phase would not be applicable. As a result, the bound on $\left|f_{3}\right|$ is much smaller than the bound on $\left|f_{2}\right|$.

At this point, it still is not clear whether or not the term with $f_{2}+f_{3}$ in eq. (4.6) is much smaller than $f_{1}$, so we will continue with a numerical example.

\subsubsection{Example}

We choose a background velocity $c_{0}=1500 \mathrm{~m} \mathrm{~s}^{-1}$, a quality factor $Q_{0}=100$, a frequency $f=15 \mathrm{~Hz}$ and a reference frequency $f_{r}=1 \mathrm{~Hz}$. These are typical values for a marine seismic survey. Fig. 4.2 plots the moduli of $f_{1}, f_{2}$, and $f_{3}$ as a function of $z / h$ for half-offsets $h$ of 50 and $1000 \mathrm{~m}$. We observe that $f_{1}$ is much larger than $f_{2}$ and $f_{3}$ over a range of depths. The contribution of $f_{3}$ can be neglected. At larger depths, the graph of $\left|f_{1}\right|$ drops because of the damping in the background medium. By the time it reaches $f_{2}$, the signal may have become too weak to be useful in practice, in the presence of noise, multiples, and so on. At the other end, where depth is small relative to offset, we can no longer neglect $f_{2}$. For a large range of depths, however, the term $f_{2}+f_{3}$ in eq. (4.6) will be small relative to $f_{1}$.

Even if $f_{2}+f_{3}$ is small compared with $f_{1}, P_{2}=\int_{-\infty}^{\infty}\left[f_{2}(z)+\right.$ $\left.f_{3}(z)\right] \operatorname{sign}(z) \mu(z) \mathrm{d} z$ does not have to be small relative to $P_{1}=$ $\int_{-\infty}^{\infty} f_{1}(z) \mu(z) \mathrm{d} z$. Figs 4.3 and 4.4 show that $f_{1}(z)$ is oscillatory, whereas $f_{2}$ is smooth and $f_{3}$ negligible. If $\mu(z)$ represents an isolated reflector localized at not too small a depth with a thickness of the order of the wavelength of the dominant signal or less, we can expect $P_{2}$ to be smaller than $P_{1}$. If, however, $\mu$ varies slowly over many oscillations of $f_{1}$, the net result may be that $P_{1}$ becomes of the same size or smaller than $P_{2}$.

We will first give a numerical example and then study the spatial Fourier transform in depth to obtain more insight into which models $\mu(z)$ will provide similar data after a weighted Hilbert transform. Fig. 4.5 shows a scattering model with a number of isolated reflectors and its transform. The recorded pressure data are displayed in Fig. 4.6, together with the difference between the data for the transformed and original model. The 

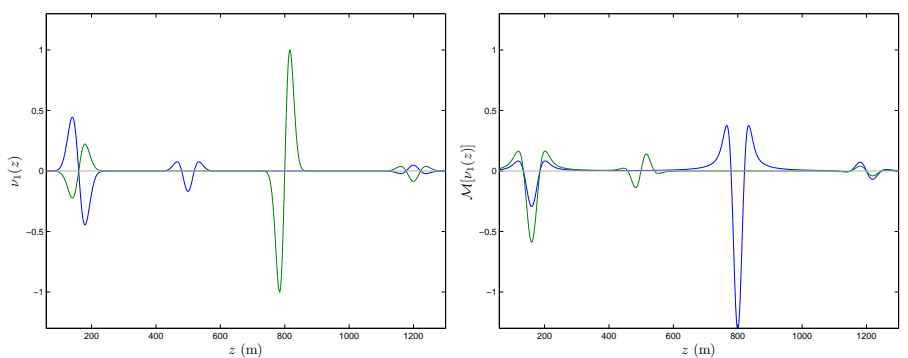

Figure 4.5: The left-hand panel shows the scatterer model $m_{1}(z)$. The blue line represents the real part of the squared slowness perturbation, the green line its imaginary part. The right-hand panel displays $\mathcal{M}_{z}\left[m_{1}\right]$, its scaled, depth-weighted Hilbert transform.
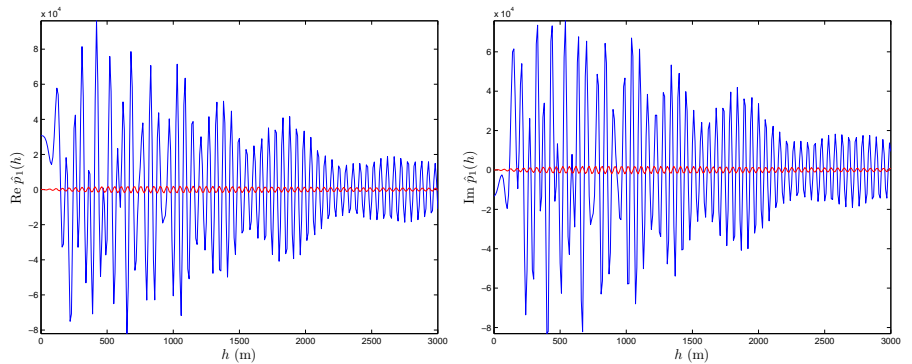

Figure 4.6: Real (left-hand panel) and imaginary (right-hand panel) part of the recorded pressure, in arbitrary units, as a function of the half-offset $h$. The blue line represents the pressure for the original model, the red one the difference between the data for the transformed and original model.

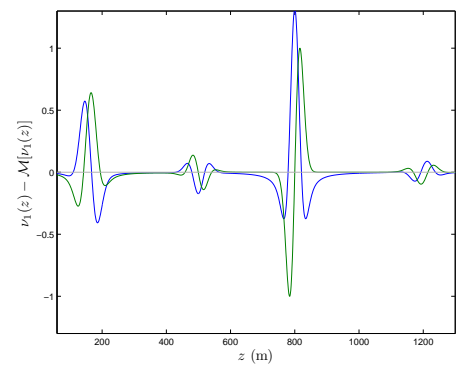

Figure 4.7: Model that directly produces the data difference in Fig. 4.6 and therefore can be considered a cloaked version of the original model in Fig. 4.5. The blue line corresponds to the real part and the green to the imaginary part of $m_{1}-\mathcal{M}_{z}\left[m_{1}\right]$. 
differences are an order of magnitude smaller than the data for the entire range of half-offsets. We found a similar behaviour for smaller and larger frequencies within the typical seismic frequency band of $8-80 \mathrm{~Hz}$ or so. This clearly illustrates that two different models, related by a scaled, depthweighted Hilbert transform, provide nearly identical data.

Note that the data difference between the two models could have been computed directly by taking a scattering model $m_{1}-\mathcal{M}_{z}\left[m_{1}\right]$. This implies that a given scattering model $m_{1}(z)$ can be 'cloaked' by replacing it with $m_{1}-\mathcal{M}_{z}\left[m_{1}\right]$, shown in Fig. 4.7 for the current example.

\subsubsection{Fourier representation}

To learn more about which scattering models provide more or less the same data before and after a weighted Hilbert transform, we consider the Fourier transform in depth.

The spatial Fourier transform $\hat{\mu}(\kappa)$ as a function of wavenumber $\kappa$ is defined in such a way that $\mu(z)=(2 \pi)^{-1} \int_{-\infty}^{\infty} \hat{\mu}(\kappa) e^{\mathrm{i} \kappa z} \mathrm{~d} \kappa$. Note that the use of the hat in the notation is potentially confusing, as we used it earlier for the temporal Fourier transform. We can write

$$
\int_{-\infty}^{\infty} f_{1}(z) \mu(z) \mathrm{d} z=\int_{-\infty}^{\infty} \hat{\mu}(\kappa) \mathcal{F}(\kappa) \mathrm{d} \kappa,
$$

where

$$
\mathcal{F}(\kappa)=\frac{1}{2 \pi} \int_{-\infty}^{\infty} f_{1}(z) e^{\mathrm{i} \kappa z} \mathrm{~d} z=-h k_{0} H_{0}^{(1)}(h \eta) / \eta,
$$

with $\eta=\sqrt{4 k_{0}^{2}-\kappa^{2}}$. This is a generalization of eq. (3.914) in Gradshteyn and Ryzhik (1965). Here $H_{0}^{(1)}(\zeta)$ is a Hankel function of the first kind. Note that $\mathcal{F}(\kappa)=\mathcal{F}(-\kappa)$ for real $\kappa$. Also, $\hat{f}_{1}(\kappa)=2 \pi \mathcal{F}(\kappa)$. Recall that $\operatorname{Re} k_{0}>0$ and $\operatorname{Im} k_{0}>0$.

In the Fourier domain, the Hilbert transform amounts to multiplication by $-i \operatorname{sign}(\kappa)$. The Fourier transform of $f_{4}(z)=i \operatorname{sign}(z) \mathcal{H}_{z}\left[f_{1}\right]$ is

$$
\hat{f}_{4}(\kappa)=-\mathrm{i} \mathcal{H}_{\kappa}\left[\hat{f}_{1}(\kappa) \operatorname{sign}(\kappa)\right] .
$$


Because we have assumed that $\mu(z)=0$ for $z<z_{m}$, with $z_{m}>0$, we should have $\mu(z)=H(z) \mu(z)$, where $H(z)$ is the Heaviside or unit step function. This results in $\hat{\mu}=-i \mathcal{H}_{\kappa}[\hat{\mu}]$ and

$$
\int_{-\infty}^{\infty} \hat{f_{4}}(\kappa) \hat{\mu}(\kappa) \mathrm{d} \kappa=\int_{-\infty}^{\infty} \hat{f_{1}}(\kappa) \hat{\mu}(\kappa) \operatorname{sign}(-\kappa) \mathrm{d} \kappa
$$

If $f_{4}(z) \simeq f_{1}(z)$, we find that

$$
\int_{-\infty}^{\infty} \hat{f_{1}}(\kappa) \hat{\mu}(\kappa) \operatorname{sign}(-\kappa) \mathrm{d} \kappa \simeq \int_{-\infty}^{\infty} \hat{f_{1}}(\kappa) \hat{\mu}(\kappa) \mathrm{d} \kappa
$$

This means that the recorded pressure hardly depends on the positive wavenumbers of $\hat{\mu}(\kappa)$.

As an illustration, Fig. 4.8 displays the Fourier transforms of $f_{1}$ and $f_{4}$ for a half-offset of $50 \mathrm{~m}$. The other parameters are the same as before. The real part has large peaks at $\kappa= \pm \operatorname{Re} 2 k_{0}$, which is the wavelength of the scattering model to which the wavefield is most sensitive. The functions $\hat{f}_{1}(\kappa)$ and $\hat{f}_{4}(\kappa)$ agree quite well except for small $\kappa$, where a singularity in $\hat{f}_{4}(\kappa)$ appears. Fig. 4.9 shows the same functions at a half-offset of $1000 \mathrm{~m}$. The singularity at $\kappa=0$ is prominent. At this larger offset, the wavefield is sensitive to a wider range of wavelengths, involving the less oscillatory components of the scattering model. This fact was exploited by Sirgue and Pratt (2004) to reduce the number of frequencies required to obtain sufficient wavelength coverage in frequency-domain full waveform inversion.

From these figures, we learn that the effect on the recorded data of the difference between $\hat{f}_{1}(\kappa)$ and $\hat{f}_{4}(\kappa)$ will be small if $\hat{\mu}(\kappa)$, the Fourier transform of the scattering model, does not contain small wavenumbers. This means that $\mu(z)$, or $m_{1}(z)$, should be sufficiently oscillatory as a function of depth. Since $\mu(z)$ was defined as the depth-weighted difference between a rough Earth model and its smoothed version, is will be oscillatory by construction. As seismic data are band-limited, linearized inversion or migration will only construct the oscillatory components of the scatterers the boundaries between different types of rock. In that case, the ambiguity will show up. We will consider this next. 

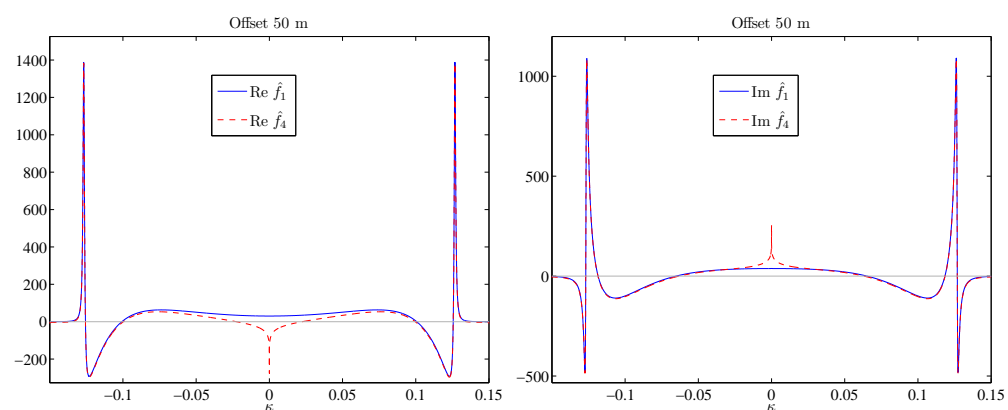

Figure 4.8: Fourier transforms of $f_{1}(z)$, the kernel between the scattering model and the recorded pressure, and $f_{4}(z)$, its weighted Hilbert transform, at a half-offset of $50 \mathrm{~m}$. The left-hand panel shows the real part of these functions, the right-hand panel the imaginary part. The functions $\hat{f}_{1}(\kappa)$ and $\hat{f}_{4}(\kappa)$ are nearly identical at larger wavenumbers $\kappa$. The peaks in the real part occur near $\kappa= \pm \operatorname{Re} 2 k_{0}$.
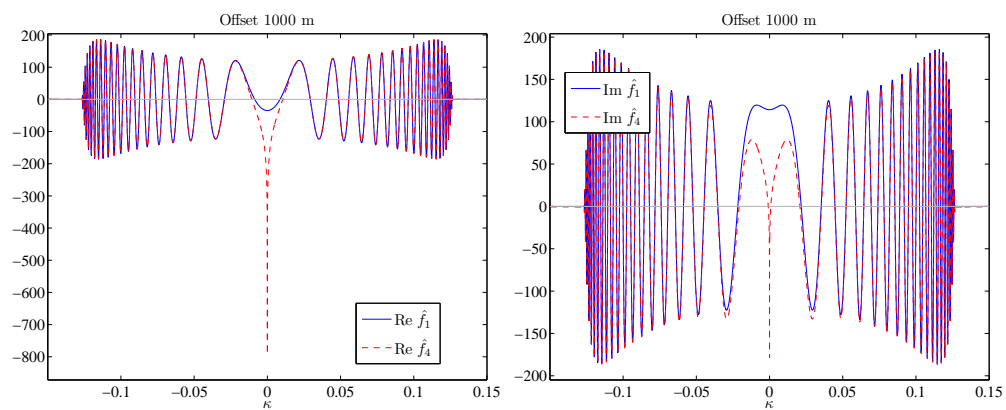

Figure 4.9: As Fig. 4.8, but for a half-offset of $1000 \mathrm{~m}$. Again, the functions $\hat{f}_{1}(\kappa)$ and $\hat{f_{4}}(\kappa)$ are nearly identical at larger wavenumbers $\kappa$. The differences at small wavenumbers are substantial. 


\subsubsection{Migration}

Given measured data, we can reconstruct the reflectivity $m_{1}(z)$ by solving the inverse problem. In geophysics, this operation is called migration. Migration is usually implemented as a single iteration step for a gradientbased minimization method with a suitable weighting or preconditioning, based on the diagonal of the Hessian of the inverse problem (Beylkin, 1985; Docherty, 1991; Gray, 1997; ten Kroode et al., 1998; Chavent and Plessix, 1999; Shin et al., 2001; Plessix and Mulder, 2004).

Consider the least-squares error

$$
J=\frac{1}{2} \sum_{h, \omega}\left|\hat{p}_{1}(\omega, h)-\hat{p}_{1}^{\mathrm{obs}}(\omega, h)\right|^{2},
$$

where $\hat{p}_{1}=F m_{1}$ are modelled data and $\hat{p}_{1}^{\text {obs }}$ observed data for receivers located at a finite number $N_{h}$ of offsets. The linear operator $F$ is defined by eq. (4.5). The inverse problem requires the solution of $F^{\mathrm{H}} F m_{1}=F^{\mathrm{H}} \hat{p}_{1}^{\text {obs }}$. Here $F^{\mathrm{H}}$ denotes the conjugate transpose. The Hessian $F^{\mathrm{H}} F$ is singular or close to singular, as follows from the previous section.

The weighted migration result is

$$
m\left(z_{1}\right) \propto \sum_{\omega, h} \omega^{2} \int_{z_{m}}^{\infty} \frac{\left|z_{1}\right|}{z_{2}} e^{2 \mathrm{i}\left(k_{0} r_{2}-k_{0}^{*} r_{1}\right)} m_{1}\left(z_{2}\right) \mathrm{d} z_{2} .
$$

Here $r_{k}=\sqrt{z_{k}^{2}+h^{2}}$ for $k=1,2$. The asterisk denotes the complex conjugate. The weighted Hilbert transform, $\mathcal{H}_{z}\left[m /\left|z_{1}\right|\right]$, involves $\mathcal{H}_{z}\left[f_{1}^{*}\right]$. Therefore, $\mathcal{H}_{z}\left[m /\left|z_{1}\right|\right] \simeq \mathrm{i}\left(m /\left|z_{1}\right|\right)$. This shows that $m /\left|z_{1}\right|$ has a minimum-norm property in the following sense.

Among all linear combinations $(1-\theta) \mu-\theta \mathrm{i} \mathcal{H}[\mu]$ with complex $\theta$, the one with $\theta=\frac{1}{2}$ has the smallest norm. To prove this, we start with the norm of a complex-valued function $a(z)$ : $\|a\|=\left[\int_{-\infty}^{\infty}|a|^{2} \mathrm{~d} z\right]^{1 / 2}$. The Hilbert transform of $a(z)$ is abbreviated as $\breve{a}=\mathcal{H}[a]$ and we set $b=-\mathrm{i} \breve{a}$. Then $b_{r}=\operatorname{Re} b=\breve{a}_{i}=\operatorname{Im} \breve{a}$ and $b_{i}=\operatorname{Im} b=-\breve{a}_{r}=-\operatorname{Re} \breve{a}$. The Hilbert transform has the property that $\left\|a_{r}\right\|=\left\|\breve{a}_{r}\right\|$ and $\left\|a_{i}\right\|=\left\|\breve{a}_{i}\right\|$. Also, $\int_{-\infty}^{\infty} a_{r} b_{i} \mathrm{~d} z=$ $-\int_{-\infty}^{\infty} a_{r} \breve{a}_{r} \mathrm{~d} z=0$ and $\int_{-\infty}^{\infty} a_{i} b_{r} \mathrm{~d} z=\int_{-\infty}^{\infty} a_{i} \breve{a}_{i} \mathrm{~d} z=0$. Let $c=(1-\theta) a+\theta b$ 
and choose $\theta=\frac{1}{2}+\beta$. Using the properties mentioned above, we find that $\|c\|^{2}=\frac{1}{4}\|a+b\|^{2}+|\beta|^{2}\|b-a\|^{2}$, which is smallest for $\beta=0$.

It is easy to show that the linear combination with the smallest norm, $\mu_{0}=\frac{1}{2}(\mu-\mathrm{i} \mathcal{H}[\mu])$, obeys $\mu_{0}=-\mathrm{i} \mathcal{H}\left[\mu_{0}\right]$. As we found earlier that this relation approximately holds for $m /\left|z_{1}\right|$, we conclude that migration of measured data provide a reconstruction of the model that approximately has this minimum-norm property.

A similar conclusion can be reached by considering the spatial Fourier transform discussed earlier in Section 4.3.4. If the ambiguity occurs, the recorded pressure is fairly insensitive to the positive wavenumbers of $\hat{\mu}(\kappa)$ according to eq. (4.8). We then can choose $\hat{\mu}(\kappa)=0$ for $\kappa>0$ without significantly changing the data. This implies $\hat{\mu}(\kappa)=-\operatorname{sign}(\kappa) \hat{\mu}(\kappa)$, so $\mu=-\mathrm{i} \mathcal{H}_{z}[\mu]$. The result is the same as the minimum-norm solution obtained by migration. The ambiguity can be removed by requiring the scattering model to be purely real-valued or purely imaginary. In the Fourier domain, a purely real-valued scattering model is obtained by setting $\hat{m}(\kappa)=\hat{m}^{*}(-\kappa)$ for positive $\kappa$, whereas the choice $\hat{m}(\kappa)=-\hat{m}^{*}(\kappa)$ leads to a purely imaginary scattering model.

\subsection{2-D numerical example}

So far, we have considered a simple 1-D model problem with horizontally layered scatterers in a 3-D homogeneous background and found that two different models provide nearly the same data. We wondered to what extent this conclusion holds in a more complex background model with dipped reflectors. We therefore generated synthetic data with a 2-D frequency-domain finite-difference code ( $\varnothing$ stmo et al., 2002; Mulder and Plessix, 2004), using the Born approximation, and compared data for a given and for a transformed reflectivity model.

The transformation had to be slightly modified to account for the fact that the 2-D data represent the response of a line-source. The asymptotic result for a homogeneous model with layered scatterers is

$$
\hat{p}_{1}^{2 D}(\omega, h) \simeq \frac{(\mathrm{i}-1) \omega^{2}}{8 \sqrt{2 \pi k_{0}}} \int_{0}^{\infty} f_{1}(z) \mu(z) \frac{\left[1+(h / z)^{2}\right]^{1 / 4}}{\sqrt{z}} \mathrm{~d} z,
$$

instead of eq. (4.5). For small $h$, this suggest depth-weighting by $\sqrt{z}$ instead 

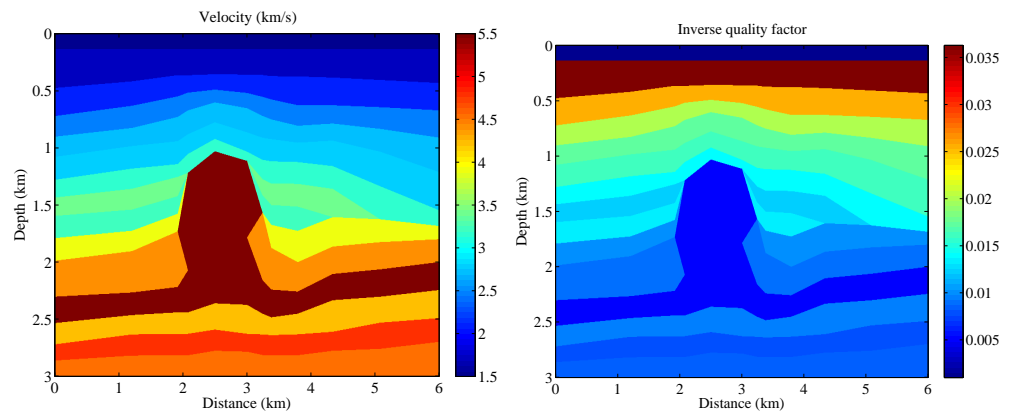

Figure 4.10: Original model with velocity $c$ (left-hand panel) and inverse quality factor $1 / Q$ (right-hand panel).
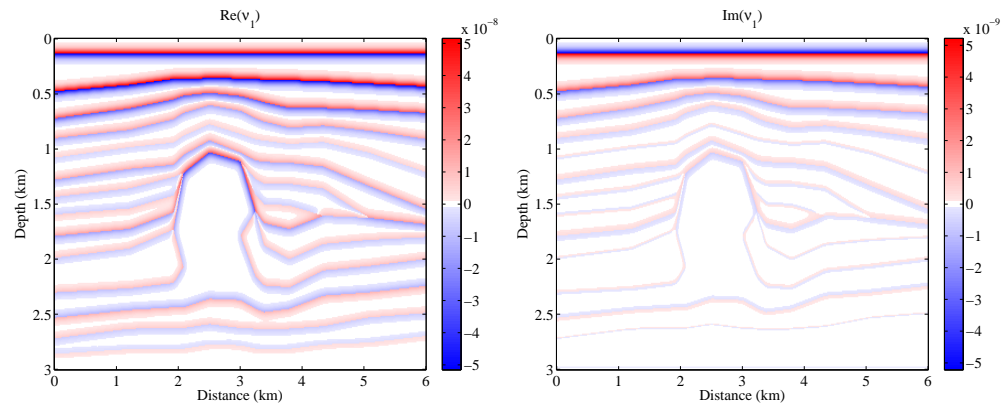

Figure 4.11: Real (left-hand panel) and imaginary (right-hand panel) part of the squared slowness perturbation $m_{1}$.
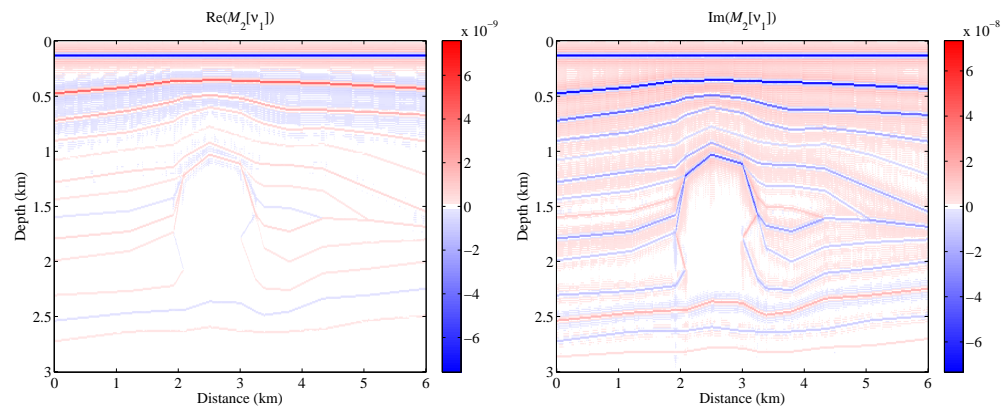

Figure 4.12: Real (left-hand panel) and imaginary (right-hand panel) part of of the transformed perturbations. 
of $z$; so, we define

$$
\mathcal{M}_{\zeta}[g](z)=\tilde{g}_{2}(z)+\tilde{g}_{2}(-z),
$$

with

$$
\tilde{g}_{2}(z)=-\mathrm{i} \sqrt{|z|} \mathcal{H}_{z}[g(z) \operatorname{sign}(z) / \sqrt{|z|}] .
$$

Fig. 4.10 shows the velocity and inverse quality factor for a simple saltdome model. We smoothed the complex-valued squared slowness and obtained the perturbation $m_{1}$ as the difference between the original and smoothed model. Fig. 4.11 displays the real and imaginary part of the perturbation. Fig. 4.12 depicts $\mathcal{M}_{\zeta}\left[m_{1}\right]$, the result of applying a weighted Hilbert transform in depth and multiplication by $-i$. A vertical crosssection is displayed in Fig. 4.13.

We computed synthetic data in both models for a shot at $x_{s}=1000 \mathrm{~m}$ and a depth $z_{s}$ of $5 \mathrm{~m}$. The receiver line had positions $x_{r}$ between 1100 and $5000 \mathrm{~m}$ at an interval of $25 \mathrm{~m}$ and $z_{r}$ at $5 \mathrm{~m}$ depth. We solved the pair of equations that represents the Born approximation of the wave equation with absorbing boundaries on all sides and transformed the frequencydomain data to the time domain with a suitable zero-phase wavelet having most of its energy between 6 and $27 \mathrm{~Hz}$. Fig. 4.14 shows the reflection data and the difference between data for the transformed and for the original model, using the same scale. The data are clipped, and the colour scale emphasizes small amplitudes. Weak reflections generated by the absorbing boundaries and aliased in time are visible. The data agree remarkably well, as can also be seen in Fig. 4.15. Only the first strong seabottom reflection and refractions at large offsets for reflectors at shallower depths show a large difference, as do the reflections from the more strongly dipped interfaces. 

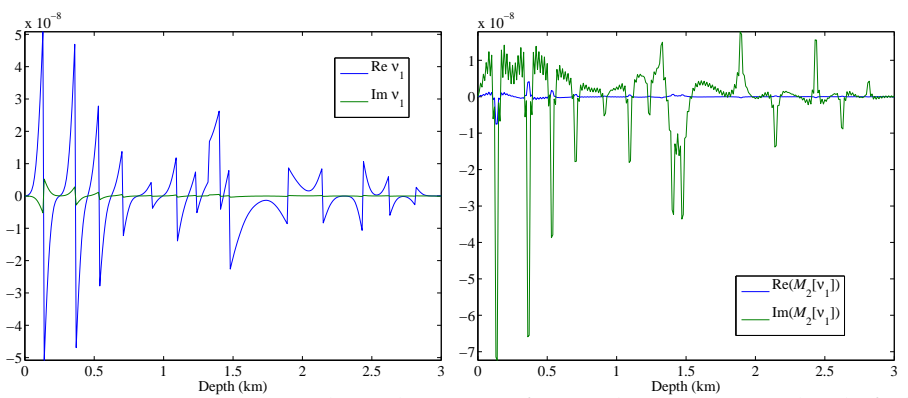

Figure 4.13: Cross-sections at $2 \mathrm{~km}$ distance from the origin. The left-hand panel shows the real (blue) and imaginary (green) part of the scatterers, the right-hand panel shows the scatterers after the depth-weighted Hilbert transform.
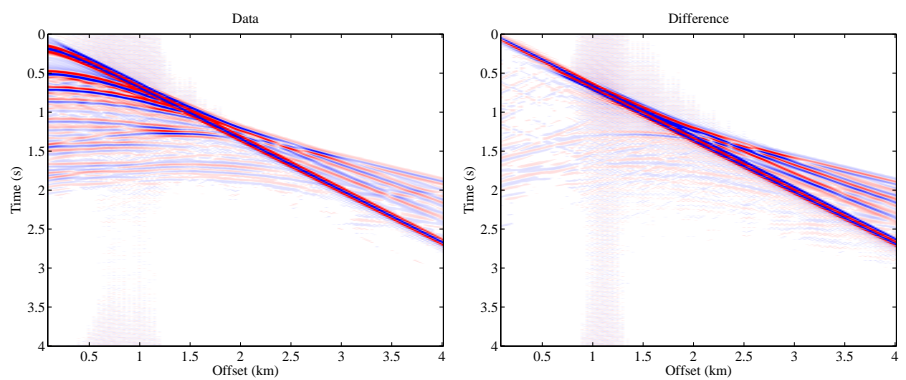

Figure 4.14: The data (left-hand panel) and the difference (right-hand panel) between data generated in the transformed and in the original model, both with the same scale. We applied a clip at 5 per cent of the maximum absolute value of the data in the left-hand panel.

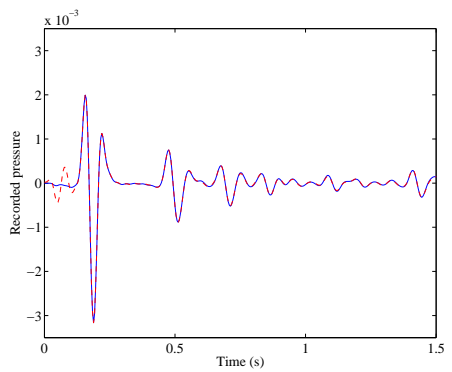

Figure 4.15: Single trace at $100 \mathrm{~m}$ offset. The blue line represents the data for the original model, the red dashed line for the transformed one. 

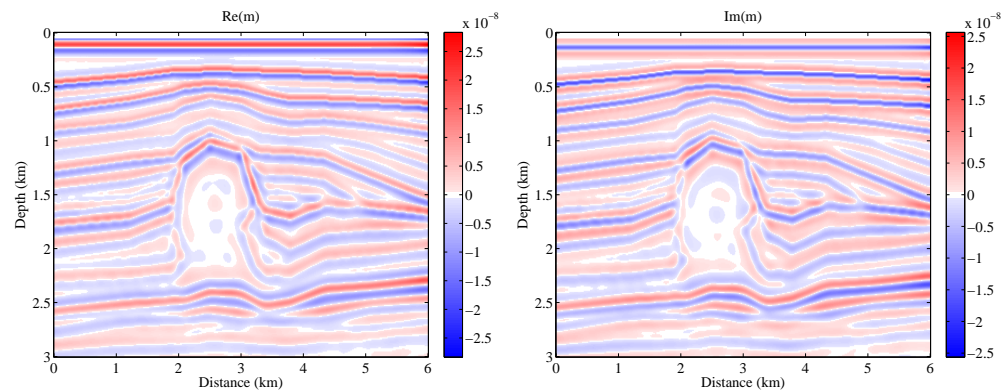

Figure 4.16: Real (left-hand panel) and imaginary (right-hand panel) part of the migration result.
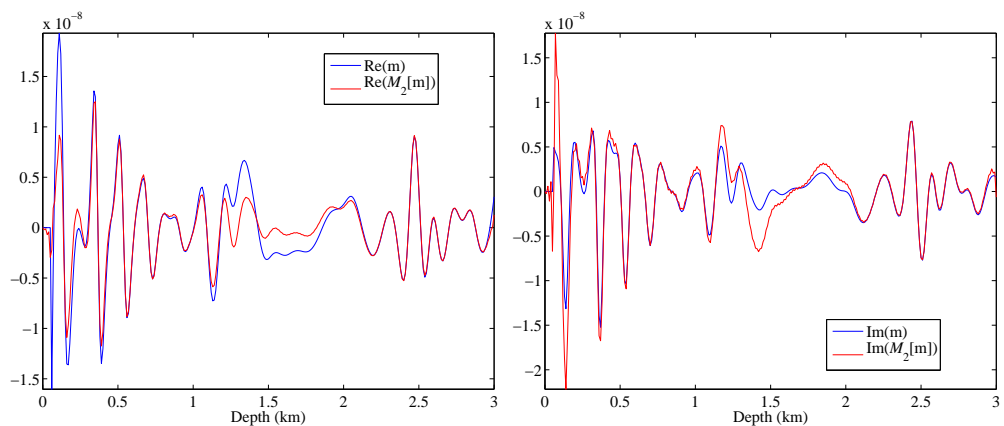

Figure 4.17: Cross-sections of the migration image at $2 \mathrm{~km}$ distance from the origin. The blue line represents the result for the original model, the red line for the transformed one. The real part is shown on the left-hand panel, the imaginary part on the right-hand panel. 
We carried out a true-amplitude two-way wave-equation migration (Plessix and Mulder, 2004) of a synthetic marine data set generated for the same 2-D model as before. Fig. 4.16 shows the real and imaginary part of the result. Fig. 4.17 displays a vertical cross-section of the 2-D 'trueamplitude' image at a distance of $2 \mathrm{~km}$ from the origin. We observe that the real and imaginary parts of the complex-valued migration result $m(x, z)$ are related by $m \simeq \mathcal{M}_{z}[m]$, as in the simple constant-velocity layered case. Discrepancies occur in the shallowest part and at depths around $1.5 \mathrm{~km}$. The first is related to the strong reflections off the sea bottom where the depth-offset ratio $z / h$ is small. Fig. 4.2 shows that the approximate equality no longer holds in that case. The second discrepancy around a depth of $1.5 \mathrm{~km}$ is caused by the presence of the nearby steeply dipping salt flank. Apart from these two differences, the figures agree with the fact that the migration selects the minimum-norm scattering model of the kind that was explained in Section 4.3.5 for horizontal reflectors. This result appears to also hold for slightly dipping reflectors.

As mentioned before, the result can be made unique by imposing constraints. If the result is assumed to be free of attenuation scattering, we can simply select the real part. If we assume that only attenuation scattering is present, we have to select the imaginary part. Without such constraints, inverting for both at the same time in a linearized sense will be problematic.

\subsection{Discussion}

We have shown that two different scattering models provide almost identical data. This implies that it, in practice, it will be impossible to uniquely determine both the velocity and attenuation perturbations from the data, unless with additional constraints. The transformed model, for instance, when added to the background model, corresponds to unphysical values of the perturbed attenuation, both in size and sign. Suitable constraints on the admissible solution will reduce, but possibly not remove, the ambiguity. Requiring the result to be without attenuation will remove the ambiguity, as will the constraint that the scattering is caused by attenuation perturbations only.

Our result seems to contradict conclusions on visco-acoustic inversion by several authors, including ourselves; so, some comments are in place. 
The existence of non-radiating sources (Bohm and Weinstein, 1948; Goedecke, 1964; Bleistein and Cohen, 1977; Devaney, 1978; Devaney and Marengo, 1998) can be related the non-uniqueness in scattering by noting that the product of the incoming wavefield with the scatterer potential acts as a secondary source (Devaney and Sherman, 1982; Hoenders, 1997; Devaney, 2004). According to Devaney and Sherman (1982), the non-uniqueness in scattering should disappear if multiple experiments are performed, for instance, at more than a single offset.

Ribodetti et al. (1995) and Hak and Mulder (2008a) consider deltafunction type perturbations or point scatterers. Its Fourier transform in depth contains long-wavelength components, including the zero wavelength. As demonstrated in Section 4.3.4, the ambiguity disappears for small wavenumbers that correspond to long wavelengths and linearized inversion should therefore be feasible. For scatterers that resemble the first or higher derivative in $z$ of a delta-function or Gaussian with small width, the long-wavelength components are small and the ambiguity should appear. This happens in the 2-D example presented here. The sea bottom is an exception, as are shallow refractions at large offsets and reflections of dipping interfaces.

Ribodetti et al. (1995) demonstrated that density, velocity, and attenuation perturbations can be recovered by inversion, but they only consider perturbations in one of these parameters at a time. With the constraint that the inverted parameter is either real or imaginary, the ambiguity is removed. Similarly, Blanch and Symes (1994) only consider a perturbation of the bulk modulus or bulk modulus and density (Blanch and Symes, 1995) in a visco-acoustic background model.

Ribodetti and Virieux (1998) presented linearized inversion results for scattering by blocky models. The Hilbert transform of a square wave has logarithmic singularities at positions corresponding to the ramps of the square wave. Such singularities should be visible as large peaks. Indeed, the reconstructed attenuation profiles in the paper by Ribodetti and Virieux (1998) sometimes show peaks at the jumps, rather than the underand overshoots next to these jumps, which are typical for least-squares inversion of blocky models in the real-valued case. Also, Innanen and Weglein (2007) consider blocky models that contain long spatial wavelengths, and can invert for the scattering model parameters.

We should emphasize that our observations do not relate to attenuation estimates from diving waves or direct arrivals in crosswell or VSP 
transmission data. These provide a characterization of the background model.

\subsection{Conclusion}

We have demonstrated that different scattering models provide almost the same data if attenuation scattering is included. The ambiguity occurs for scatterers with small dip that are located sufficiently deep and that have an oscillatory character. The last condition is typically met when migrating seismic data. Because the data are band-limited, the migration algorithm will only be able to reconstruct the difference between a rough Earth model and its smoothed version. This difference is oscillatory by definition. As a consequence, attenuation scattering imaging will be nearly impossible without imposing additional constraints. The ambiguity can be removed by requiring the scatterer to have no attenuation at all, or to be purely attenuative. 


\title{
Chapter 5
}

\section{Migration for velocity and attenuation perturbations}

\begin{abstract}
Migration maps seismic data to reflectors in the Earth. Reflections are not only caused by small-scale variations of the velocity and density, but also of the quality factor that describes attenuation. We investigated scattering due to velocity and attenuation perturbations by computing the resolution function or point-spread function in a homogeneous background model. The resolution function is the migration image of seismic reflection data generated by a point scatterer. We found that the resolution function mixes velocity and attenuation parameters perturbations to the extent that they cannot be reconstructed independently. This is true for a typical seismic setting with sources and receivers at the surface and a buried scatterer. As a result, it will be impossible to simultaneously invert for velocity and attenuation perturbations in the scattering approach, also known as the Born approximation.

We proceeded to investigate other acquisition geometries that may resolve the ambiguity between velocity and attenuation perturbations. With sources and receivers on a circle around the scatterer, in 2D, the ambiguity disappears. It still shows up in a cross-well setting, although the mixing of velocity and attenuation parameters is less severe than in the surface-to-
\end{abstract}


surface case. We also consider illumination of the target by diving waves in a background model that has velocity increasing linearly with depth. The improvement in illumination is, however, still insufficient to remove the ambiguity.

\subsection{Introduction}

Migration maps seismic data recorded at the Earth's surface into an image of the subsurface. Although qualitative structural images are often acceptable, quantitative characterisation of a scatterer's reflection and attenuation properties may enable a distinction between fluid-bearing and gas- or air-filled geological formations. For migration, the implicit assumption is that the data only contain primaries and that surface or interbed multiples are absent or negligible. We can generate such data with the linearised wave equation or Born approximation. In this approximation, the subsurface model is split into a part that does not produce reflections and a perturbation that generates the reflection data. One way to obtain a background model that does not generate reflections in the seismic frequency band is the choice of a smooth model. The differences between the original model that may contain hard contrasts in material properties and its smooth version can be viewed as perturbations. Migration of seismic data then provides a reconstruction of these reflectors. In this setting, impedance contrasts become velocity and density perturbations. They are not the only perturbations that create reflections. Attenuation perturbations can do that as well (e.g. Vasheghani and Lines, 2009). The formulation of the migration operator in the frequency domain naturally lends itself for imaging of velocity, density, and attenuation perturbations in a given smooth background model. The goal of this paper is to study migration in this context. For simplicity, we consider the constant-density acoustic wave equation. Impedance perturbations are thereby reduced to velocity perturbations.

From a mathematical point of view, migration can be viewed as the gradient or sensitivity of the least-squares error functional with respect to the model parameters (Lailly, 1983; Tarantola, 1984). The error functional is the sum of squared differences between modelled and observed data. A gradient-based optimisation algorithm can attempt to find the model that best explains the data. Because seismic data are band-limited, 
this minimisation problem is plagued by spurious local minima, making full waveform inversion difficult except when a very good initial model is available, so that gradient-based optimisation will end up in a nearest minimum that is actually the global minimum, or when waveform tomography can be performed, in a cross-well setting or with diving waves from the surface of the Earth. See, for example, papers by Tarantola (1984, 1986), Mora (1987, 1988, 1989), Crase et al. (1990, 1992), Pratt (1999), Hicks and Pratt (2001), Shin et al. (2001), Shipp and Singh (2002), Operto et al. (2004), Barnes et al. (2008), Royle and Singh (2008), and many others. The least-squares problem can be simplified by linearisation of the inverse problem. This is equivalent to using the Born approximation for the forward modelling problem. The model parameters then become, for instance, the impedance perturbations of the background model. Minimisation of the least-squares functional still makes sense in this context. The data should then be restricted to primaries. The result of the minimisation will be a band-limited reconstruction of the reflectivity, deconvolved for the source wavelet (Jin et al., 1992; Lambaré et al., 1992; Nemeth et al., 1999; Østmo et al., 2002; Mulder and Plessix, 2004; Plessix and Mulder, 2004; Gélis et al., 2007). Algorithmically, this approach resembles full waveform inversion. However, as the inverse problem is now linear in the model parameters, a preconditioned conjugate-gradient method suffices to find the best reflectivity model.

Application of the conjugate-gradient method has the advantage that the convergence of the method will not be affected by the presence of nullspace components. A suitable preconditioner will accelerate convergence to the model that best explains the data. If there are null-space components, the conjugate-gradient method without a preconditioner will convergence to the minimum-norm solution. With a preconditioner, the method will convergence to a different solution that has a smallest weighted norm. The preconditioner implicitly defines the weighting. The inverse of the Hessian, the second derivative of the least-squares cost functional with respect to the model parameters, is the best preconditioner, but is computationally out of reach for large-scale problems. In spite of that, it was considered by Pratt et al. (1998). In this paper, we will compute and use it to better understand attenuation imaging.

Estimating attenuation is usually restricted to the background model. Causse et al. (1999), Hicks and Pratt (2001), and Innanen and Weglein (2007) present examples for nonlinear inversion. Incorporation of attenuation in 
the reflectivity was considered by Ribodetti et al. (1995) and Ribodetti and Virieux (1998). The goal of the present paper is to obtain insight in the problems that surround the imaging of attenuation perturbations. A method for the estimation of multiple parameters was presented by Kennett et al. (1988) and Kennett and Sambridge (1998), but this approach will not be considered here. Mulder and Hak (2009) show that simultaneous imaging of velocity and attenuation perturbation with the Born approximation of the constant-density visco-acoustic wave equation leads to an ambiguity: the data can be equally well explained by a velocity perturbation or by an attenuation perturbation. There exists a simple transformation between these two models for 1D, horizontally layered scattering models in a constant background. Here, we elaborate on the subject by considering several acquisition geometries with the purpose of finding one that alleviates or removes the ambiguity. We consider simple 2D examples for which the Green functions are available and the full Hessian can be readily computed. In earlier publications (Hak and Mulder, 2008a,b), we considered various non-diagonal preconditioners for the linearised inverse problems, but once we realised the existence of the ambiguity mentioned above, we concluded that such preconditioning will never help in providing a proper answer.

The goal of our paper is to study the occurrence of the ambiguity between velocity and attenuation perturbations for two simple background models and investigate if there exist acquisition geometries that provide sufficient illumination to reduce its effect or even completely remove it. We will stick to the Born approximation, despite its limited validity, because of its natural correspondence to seismic migration. In Section 5.2, we formulate the problem of estimation velocity and attenuation parameters. Section 5.3 describes the migration of surface data for a buried point scatterer in a homogeneous background model and the result of its reconstruction based in the pseudo-inverse of the Hessian. We proceed in Section 5.4 with a circular and a cross-well acquisition in order to investigate if this will remove the ambiguity. Also, a velocity model that increases linearly with depth is considered, because it will lead to diving waves that improve the illumination of the target as long as it is not located too deeply (Mulder and Plessix, 2008). Finally, we summarise and discuss the results. 


\subsection{Problem statement}

The constant-density visco-acoustic wave equation in the frequency domain reads

$$
-\omega^{2} \tilde{m} \tilde{p}-\Delta \tilde{p}=S,
$$

with pressure $\tilde{p}(\omega, x)$ depending on position $x$ and angular frequency $\omega=$ $2 \pi f$ at frequency $f$, source term $S(\omega, x)$, and model parameters (Aki and Richards, 2002)

$$
\tilde{m}(x)=\frac{1}{v^{2}}=\frac{1}{c^{2}}\left[1-\frac{2}{\pi Q} \log \left(f / f_{r}\right)+\frac{\mathrm{i}}{Q}\right] .
$$

The sound speed is $c(x)$. The quality factor $Q(x)$ describes attenuation. The logarithmic term is required for causality and is defined relative to some reference frequency $f_{r}$. Because we are interested in imaging reflectors or scatterers, we use the Born approximation. Let $\tilde{m}=m_{b}+m$, where $m_{b}(x)$ represents a smooth background velocity model that does not produce significant reflections in the seismic frequency band. The Born approximation is represented by the pair of equations

$$
\begin{aligned}
-\omega^{2} m_{b} p_{b}-\Delta p_{b} & =S, \\
-\omega^{2} m_{b} p-\Delta p & =\omega^{2} m p_{b} .
\end{aligned}
$$

The background pressure wavefield is denoted by $p_{b}$ and the reflection data by $p$. For simplicity, we will assume that $m$ is independent of $\omega$, so the frequency-dependent correction term is ignored in the scattering model, but not in the background model. Hence, a perturbation of the real part of $m$ relates to a velocity perturbation, $\delta\left(c^{-2}\right)=c^{-2}-c_{b}^{-2}$, whereas a perturbation of the imaginary part of $m$ relates to a perturbation of a combination of the velocity and the quality factor, $\delta\left(c^{-2} Q^{-1}\right)=c^{-2} Q^{-1}-$ $c_{b}^{-2} Q_{b}^{-1}$. Note that for a purely imaginary perturbation, $\delta\left(c^{-2}\right)=0$ and $\delta\left(c^{-2} Q^{-1}\right)=c_{b}^{-2}\left(Q^{-1}-Q_{b}^{-1}\right)$, meaning that we only have a perturbation in the quality factor that describes the attenuation.

For a delta-function source at position $x_{s}$ with wavelet $w(\omega), S=$ $w(\omega) \delta\left(\boldsymbol{x}-\boldsymbol{x}_{s}\right)$, the solution at some receiver location $\boldsymbol{x}_{r}$ can be expressed by 
equation (3.2.1) of Bleistein et al. (2000), which in our notation becomes

$$
p_{r(s)}=p\left(\omega, x_{r}\right)=w(\omega) \int d x \omega^{2} G\left(\omega, x_{s}, x\right) m(x) G\left(\omega, x, x_{r}\right) .
$$

The Green function $G\left(\omega, x_{1}, x_{2}\right)$ is determined by the background model $m_{b}(\boldsymbol{x})$ and describes the wavefield at position $x_{2}$ when equations (5.2) and (5.3) are solved for a delta-function source at $x_{1}$ with unit wavelet in the frequency domain. Equation (5.4) represents a linear map from $m(x)$ to $p_{r(s)}$, which we represent as $\mathbf{p}=\mathrm{Fm}$. Here $\mathbf{p}$ is the seismic data set with primary reflections only, for all shots, labelled by $s$, receivers $r(s)$, and frequencies $\omega$. Note that $F$ depends on the background model $m_{b}$, the acquisition, and the source wavelet.

Migration map the data back to the subsurface according to $g=F^{H} p$. The superscript $(\cdot)^{\mathrm{H}}$ denotes the complex conjugate transpose. For a given model $\mathbf{m}$, this implies that the migration image can be expressed as $\mathbf{g}=\mathrm{Hm}$, with $\mathrm{H}=\sum_{\omega} \mathrm{F}^{\mathrm{H}} \mathrm{F}$. The operator $\mathrm{H}$ describes how a given reflectivity model is transformed into an image after forward modelling and migration, given a certain acquisition. If $m(x)=\delta\left(\boldsymbol{x}-\boldsymbol{x}_{p}\right)$ is a delta-function or spike in a single position $x_{p}$, then $\mathrm{H} \delta\left(x-x_{p}\right)$ describes how closely the corresponding migration image matches its original. Several authors therefore refer to $\mathrm{H} \delta\left(\boldsymbol{x}-\boldsymbol{x}_{p}\right)$ as the point-spread function (Devaney, 1984; Gjøystdal et al., 2002) or resolution function (von Seggern, 1991; Wapenaar, 1997; Chen and Schuster, 1999; Berkhout et al., 2001; Toxopeus et al., 2004, 2008). Lailly (1983) and Tarantola (1984) showed that the gradient or sensitivity of the least-squares error functional is a migration image. If $p_{r(s)}^{\mathrm{obs}}(\omega)$ denotes the observed data and $p_{r(s)}(\omega)$ the synthetic data for some model $m(x)$, the least-squares functional is

$$
\mathcal{J}=\frac{1}{2} \sum_{\omega} \sum_{s} \sum_{r(s)}\left|p_{r(s)}(\omega)-p_{r(s)}^{\mathrm{obs}}(\omega)\right|^{2} .
$$

The gradient or sensitivity of $\mathcal{J}$ with respect to the model parameters is denoted by $g$. Its elements $g(x)$ are the derivatives of $\mathcal{J}$ with respect to the real and imaginary part of $m(x)$ in the points $x$.

Minimisation of the cost functional $\mathcal{J}$ subject to equations (5.2) and (5.3) can be accomplished with a gradient-based descent method, for instance, the conjugate-gradient method (Østmo et al., 2002). The conjugategradient method has the useful property of finding a minimum-norm 
solution if the problem has a non-empty null space. Convergence can be accelerated by using the second derivatives or Hessian of the functional with respect to the model parameters. In general, the size of the Hessian makes its computation too costly, although it can be computed for small problems (Pratt et al., 1998; Chavent and Plessix, 1999; van Houten et al., 1999; Plessix and Mulder, 2004). It is usually more efficient to replace the Hessian by an approximation of its diagonal and use the inverse of the resulting diagonal matrix as a preconditioner for the conjugate-gradient method. This is motivated by the fact that the dominant term in the Hessian is diagonal in the asymptotic limit for high frequencies (Beylkin and Burridge, 1990; ten Kroode et al., 1998) in the absence of multiples. The elements of the inverse of the diagonal approximation are sometimes called 'true-amplitude' migration weights. With such weights, just a single step of a preconditioned conjugate-gradient algorithm may already provide a result that is acceptable for further interpretation (Docherty, 1991; Gray, 1997; Chavent and Plessix, 1999; Mulder and Plessix, 2004; Plessix and Mulder, 2004). An alternative was presented by Rickett (2003) and Guitton (2004), based on migration, demigration, and remigration, followed by amplitude comparison. Symes (2008) describes further improvements. In this paper, we will actually compute the full Hessian and its pseudoinverse. For given data, this will directly provide the model at which the least-squares cost functional has its minimum.

\subsection{Resolution function for a constant model}

As a first example, we considered the point-spread or resolution function for a point scatterer in a 2D model with a constant velocity of $c_{b}=2 \mathrm{~km} / \mathrm{s}$ and quality factor $Q_{b}=100$. We included the causal correction term in equation (5.1) with $f_{r}=1 \mathrm{~Hz}$, but ignored it in the reconstruction of the scatterer, so $m(x)$ in equation (5.3) is assumed to be independent of frequency. The point scatterer, $m(x)=\delta\left(\boldsymbol{x}-\boldsymbol{x}_{p}\right)$, had a real-valued, unit amplitude and was placed at $x_{p}=0 \mathrm{~m}$ and $z_{p}=750 \mathrm{~m}$. Receivers were positions at zero depth from $x=-1900$ to $1900 \mathrm{~m}$ at a $25 \mathrm{~m}$ interval. Shots are located between $x=-1887.5$ and $1887.5 \mathrm{~m}$ at the same interval. The Green function $G\left(\omega, x_{1}, x_{2}\right)=\frac{i}{4} H_{0}^{(1)}(k r)$ with $r=\left[\left(x_{2}-x_{1}\right)^{2}+\left(z_{2}-z_{1}\right)^{2}\right]^{1 / 2}$. The Hankel function or Bessel function of the third kind $H_{0}^{(1)}(x)=J_{0}(x)+\mathrm{i} Y_{0}(x)$ is related to the Bessel functions $J_{0}(x)$ of the first kind and $Y_{0}(x)$ of the 

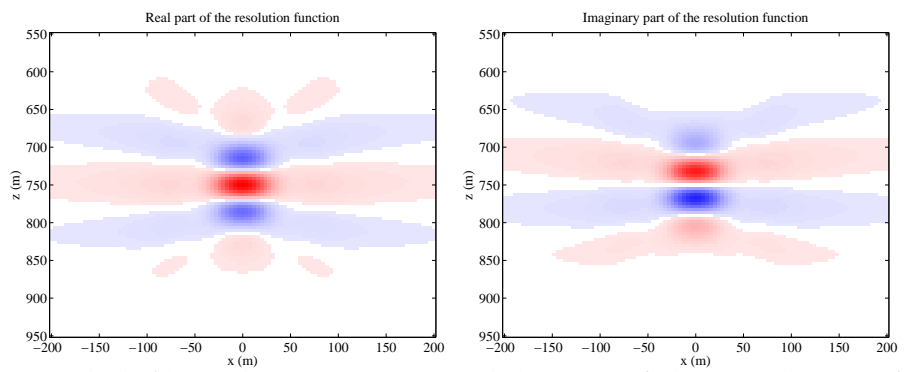

Figure 5.1: Real (left) and imaginary (right) part of the resolution function for a point scatterer at $750 \mathrm{~m}$ depth in a homogeneous background model. Colour scales are the same for both panels, with positive values in red and negative values in blue.

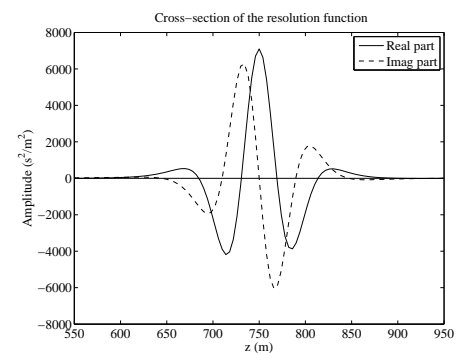

Figure 5.2: Real (drawn) and imaginary (dashed) part of the resolution function along the vertical cross-section going through the point scatterer at $750 \mathrm{~m}$ depth.

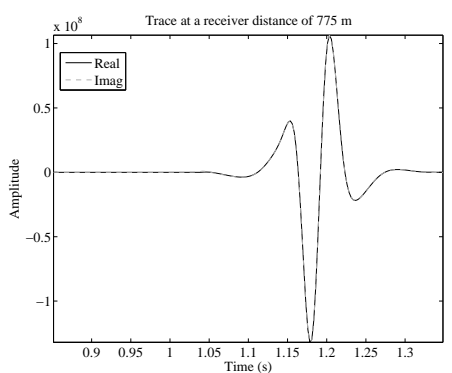

Figure 5.3: Data for a 2D scattering model. The black curve was obtained for a scattering model equal to the real part of the resolution function, the grey dashed line corresponds to $i$ times its imaginary part. The curves are practically on top of each other. 
second kind. We included a Ricker wavelet with a peak frequency of $15 \mathrm{~Hz}$ and used frequencies from 0.5 to $41 \mathrm{~Hz}$ with an interval of $0.5 \mathrm{~Hz}$. The resulting resolution function is similar to examples by, for instance, Wu and Toksöz (1987), Chen and Schuster (1999), and Berkhout et al. (2001), but now includes an imaginary part. Figure 5.1 displays its real and imaginary part. Although the original scatterer was real-valued, its resolution function or migration image has a substantial imaginary part. This can be observed in Figure 5.2, showing a vertical cross-section through the scatterer. Note that a point scatterer of the form i $\delta\left(x-x_{p}\right)$ would lead to a resolution function with an imaginary part identical to the real part of the earlier one and a real part equal to minus the imaginary part of the earlier one, as the result is linear in the scatterer model.

We took the migration result and used it to generate new data. Demigration of the real part of the migration image, displayed in the left panel of Figure 5.1, produced one frequency-domain data set. Demigration of the imaginary part of the migration image, shown in the right panel of Figure 5.1, produced another complex-valued frequency-domain data set, which we multiplied by i. We then applied a Fourier transform to obtain time-domain data. The resulting data sets turned out to be nearly identical. Figure 5.3 shows a single trace of each data set for a shot located at $x_{s}=-987.5 \mathrm{~m}$ and a receiver at $x_{r}=775 \mathrm{~m}$. We therefore have two different models that produce the same data. This suggests that, in the current example,

$$
\mathrm{F}\left\{\operatorname{Re}\left[\sum_{\omega} \mathrm{F}^{\mathrm{H}} \mathrm{F}\right] \delta\left(x-x_{p}\right)\right\} \simeq \mathrm{iF}\left\{\operatorname{Im}\left[\sum_{\omega} \mathrm{F}^{\mathrm{H}} \mathrm{F}\right] \delta\left(x-x_{p}\right)\right\} .
$$

This states that a scattering model that equals the real part of the resolution function produces nearly the same data as a model that equals i times the imaginary part of the resolution function. Appendix 5.A demonstrates that a similar approximate equality holds for a 1D, horizontally layered scattering model in a constant 3D background. The present numerical result suggests a generalisation to a $2 \mathrm{D}$ scattering model in a constant $2 \mathrm{D}$ background. So far, we have not attempted to prove equation (5.5).

Next, we consider the reconstruction of the original scattering model given the seismic data for the specific acquisition and source signature. Because $\mathrm{H}$ is singular, we have to resort to its pseudo-inverse, also known as generalised or Moore-Penrose inverse, $\mathrm{H}^{+}$, which can be computed from 
its singular-value decomposition $\mathrm{H}=\mathrm{VDV}^{\mathrm{H}}$. Here $\mathrm{D}$ is a diagonal matrix that contains the eigenvalues. Because $\mathrm{H}$ is Hermitian, the eigenvalues are real. Because $\mathrm{H}$ is the product of $\mathrm{F}$ and its conjugate transpose, these eigenvalues are non-negative. The columns of $\mathrm{V}$ contain the corresponding orthonormal eigenvectors. Therefore, $\mathrm{V}^{-1}=\mathrm{V}^{\mathrm{H}}$. The pseudo-inverse becomes $\mathrm{H}^{+}=\mathrm{VD}^{+} \mathrm{V}^{H}$, where $\mathrm{D}^{+}$contains the inverse for non-zero elements and has a zero otherwise. Because of the presence of numerical round-off errors, we assumed an eigenvalue to be zero if it was smaller than $10^{-14}$ times the largest eigenvalue. Figure 5.4 shows the eigenvalues of $H$, the elements of $D$, for the current example.

The 'best' reconstruction of the scatterer is given by $\mathbf{m}_{1}=\mathrm{H}^{+} \mathrm{H} \mathbf{m}$. Figure 5.5 displays the reconstruction $\mathbf{m}_{1}$ for a point scatterer with real-valued, unit amplitude located at $x_{p}=y_{p}=0 \mathrm{~m}$ and $z_{p}=750 \mathrm{~m}$. Again, we observe a substantial contribution in the imaginary part. To convince ourselves that this is not a result of the limited bandwidth imposed by the Ricker wavelet, we repeated the exercise for an extended scatterer, the z-derivative of Gaussian:

$$
m_{g}(x, z)=-\frac{\partial}{\partial z} \frac{1}{2 \pi \sigma_{g}^{2}} \exp \left(-\frac{\left(x-x_{p}\right)^{2}+\left(z-z_{p}\right)^{2}}{2 \sigma_{g}^{2}}\right) .
$$

We set $\sigma_{g}=13.3 \mathrm{~m}$, at one tenth of the wavelength corresponding to the peak frequency of the wavelet. Figure 5.6 shows a vertical line through the original, purely real-valued scatterer and the real and imaginary part of its reconstruction. Again, there the imaginary part has about the same amplitude as the real part.

The real and imaginary part of the resolution function are approximately related by a $z$-weighted Hilbert transform for 1D horizontally layered scattering models and also on the symmetry line through a localised scatterer. We discussed this in detail in Mulder and Hak (2009). We showed that two scattering models, related by a depth-weighted Hilbert transform, will produce approximately the same data. This implies that for the reconstruction problem, we can select any linear combination of those two models. Migration selects the one with the smallest norm, as does the pseudo-inverse of the Hessian.

Next, we will search for cases were the ambiguity can be fully or partially removed. 


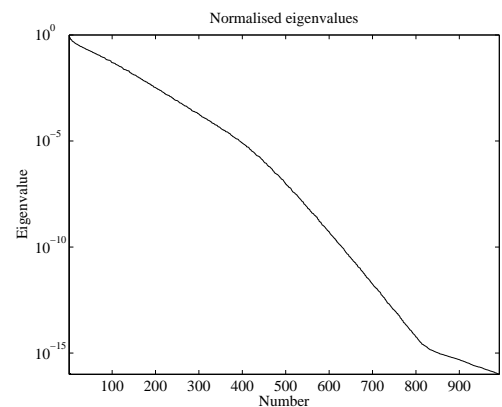

Figure 5.4: The eigenvalues of the Hessian, normalised by the largest eigenvalue. Values smaller than $10^{-16}$ are not shown.

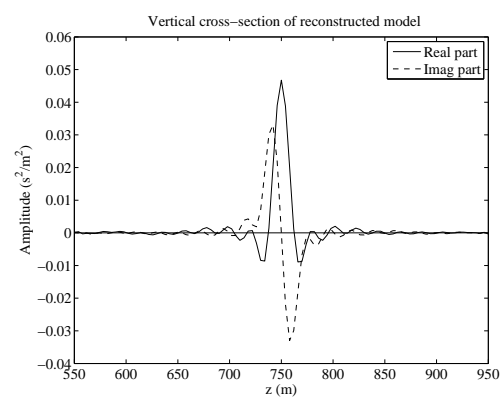

Figure 5.5: Real (drawn) and imaginary (dashed) part of a vertical cross-section through the reconstruction of the point scatterer at $750 \mathrm{~m}$ depth.

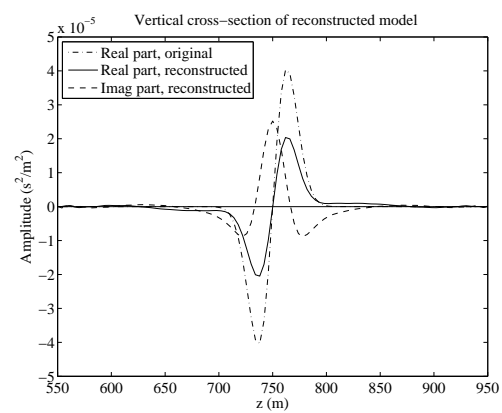

Figure 5.6: Real (drawn) and imaginary (dashed) part of a vertical cross-section through the reconstruction of an extended scatterer centred at $750 \mathrm{~m}$ depth. The dash-dotted line represents the original scatterer, which had a zero imaginary part. 


\subsection{Other acquisition geometries}

The ambiguity in the reconstruction of a scattering model shows up for a surface acquisition. We investigated if the same is true for other acquisition geometries. We start with a circle around a scattering object, continue with a cross-well example, and then consider diving waves in a velocity model that is linear in depth.

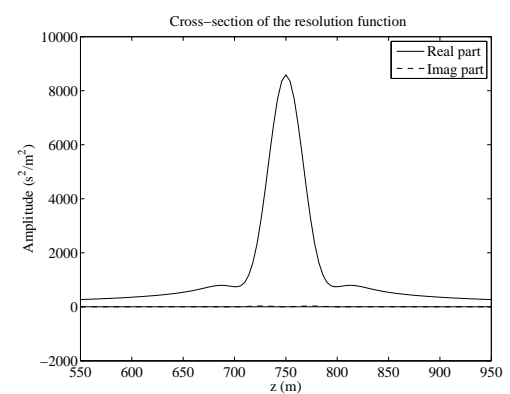

Figure 5.7: Real (drawn) and imaginary (dashed) part of the resolution function along a vertical cross-section through the point scatterer at $750 \mathrm{~m}$ depth for a circular acquisition geometry.

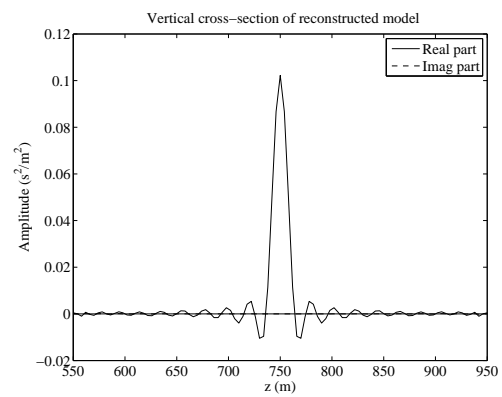

Figure 5.8: Real (drawn) and imaginary (dashed) part of the reconstructed model along a vertical cross-section through the point scatterer at $750 \mathrm{~m}$ depth for a circular acquisition geometry. 


\subsubsection{Circle}

An acquisition geometry with source and receivers on a circle surrounding the scatterer provides optimal illumination. We placed 90 sources and 90 receivers on a circle with a radius of $750 \mathrm{~m}$ around the point scatterer, interleaved at regular angles. Figure 5.7 shows a vertical cross-section of the resolution function obtained with a $15-\mathrm{Hz}$ Ricker wavelet for this acquisition. The cross-section cuts through the position of the scatterer. The resolution function has circular symmetry around this point for the chosen acquisition geometry. Its imaginary part is nearly invisible in the image. A similar section of the reconstructed model, obtained by using the pseudo-inverse of the Hessian, is displayed in Figure 5.8. Clearly, the ambiguity does not show up in this ideal setting. Ribodetti et al. (2000) found a similar result.

The high degree of symmetry leaves no room for an antisymmetric imaginary part in this example. We therefore repeated the computations for a shifted circle, centred at the same $x=0 \mathrm{~m}$ but with a shallower depth of $z=550 \mathrm{~m}$. The real part of the resolution function is quite similar to the earlier case of a circular acquisition centred around the scatterer, but its imaginary part, displayed in Figures 5.9 and 5.10, is now non-zero. The reconstructed model, however, has an negligible imaginary part and is nearly identical to the earlier result shown in Figure 5.8. Its horizontal cross-section, not shown either, has the same shape as the vertical crosssection.

\subsubsection{Cross-well data}

Placing sources and receivers all around a scatterer is difficult to realise in seismic exploration. A cross-well experiment comes close. We considered a highly symmetric acquisition geometry with two wells. Sources where placed at $x=-250 \mathrm{~m}$ and receivers at $x=+250 \mathrm{~m}$, both at depths between 0 and $1500 \mathrm{~m}$ at a $25-\mathrm{m}$ interval. The high degree of symmetry resulted in a negligible imaginary part, both for the resolution function and for the band-limited reconstruction of the point scatterer.

We also considered a less symmetric case by placing the sources at $x=-$ $250 \mathrm{~m}$ and the receivers at $x=+450 \mathrm{~m}$, with depths between 0 and $1000 \mathrm{~m}$ at a 25-m interval while keeping the point scatterer at $x=0 \mathrm{~m}$ and $z=750 \mathrm{~m}$. Figures 5.11 and 5.12 display the real and imaginary part of the resolution 


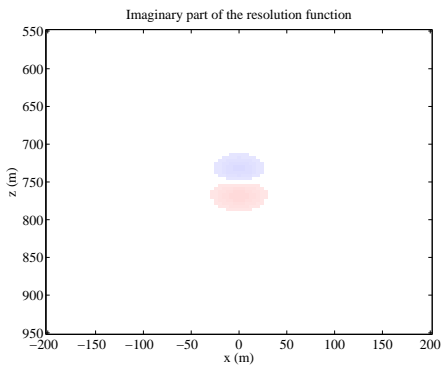

Figure 5.9: Imaginary part of the resolution function for a point scatterer at $750 \mathrm{~m}$ depth surrounded by sources and receivers on a shifted circle.

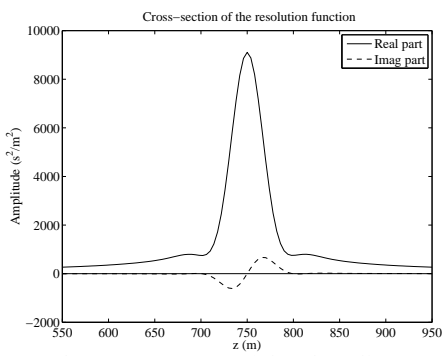

Figure 5.10: Real (drawn) and imaginary (dashed) part of the resolution function along a vertical cross-section through the point scatterer at $750 \mathrm{~m}$ depth for a shifted circular acquisition geometry.
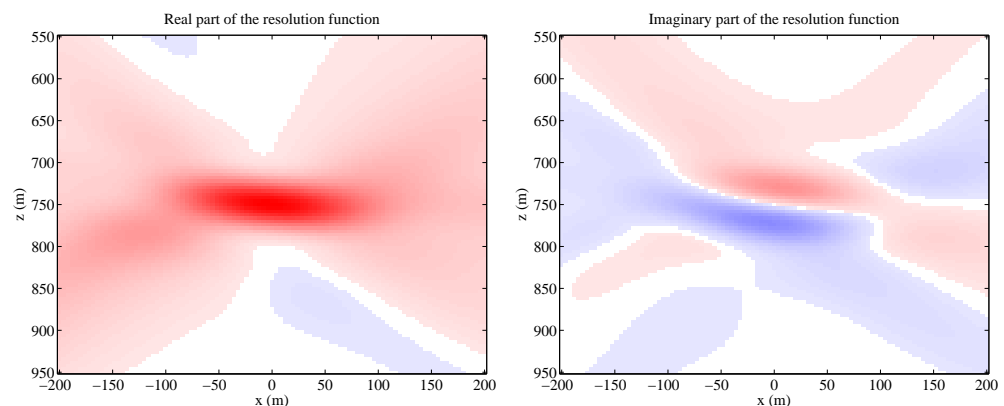

Figure 5.11: Real part (left) and imaginary part (right) of the resolution function for a point scatterer at $750 \mathrm{~m}$ depth for a cross-well configuration with sources and receivers in wells on either side of the scatterer. Colour scales are the same for both panels, with positive values in red and negative values in blue. 


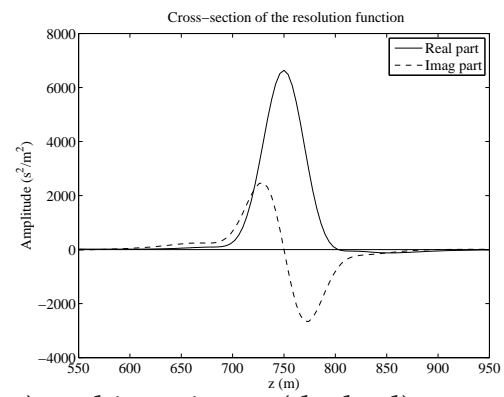

Figure 5.12: Real (drawn) and imaginary (dashed) part of the resolution function (top) along a vertical cross-section through the point scatterer at $750 \mathrm{~m}$ depth, for a cross-well acquisition geometry.
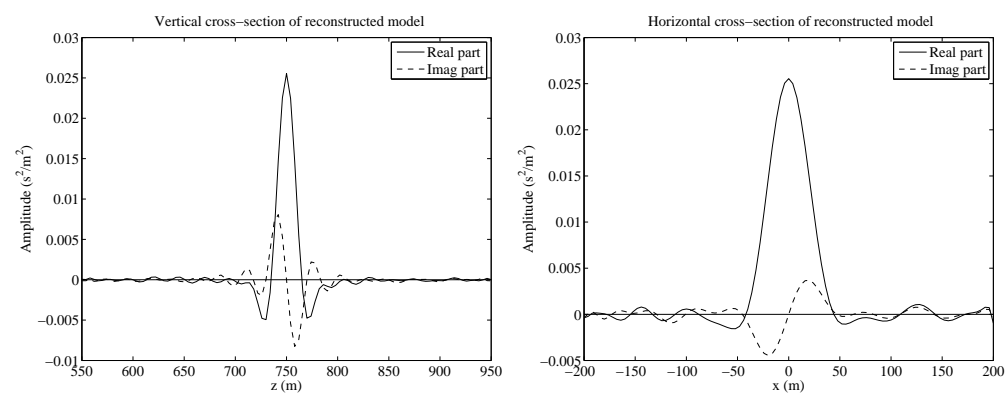

Figure 5.13: Real (drawn) and imaginary (dashed) part of the reconstructed scatterer along a vertical line (left) and horizontal line (right) through its original point scatterer position, for a cross-well acquisition geometry.

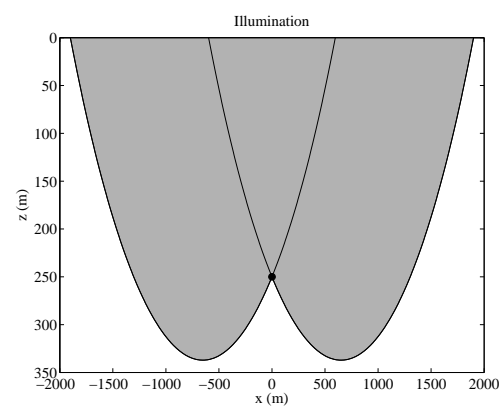

Figure 5.14: Illumination of a point scatterer in a velocity model that increases linearly with depth. 
function as well as a vertical section through the scattering point. Both the resolution function and the reconstructed parameter, shown in Figure 5.13, have a non-negligible imaginary part, although its amplitude is considerably smaller than in the case of a surface acquisition. We conclude that a cross-well setting does not completely remove the ambiguity.

\subsubsection{Diving waves}

In the above, we have seen that a circular acquisition helps to remove the ambiguity, at least for the simple case considered, and that a cross-well setting does not do so completely, although the amplitude of the erroneous part is smaller than in the case of a surface acquisition. In the last case, we only have illumination from above. If, however, the velocity increases with depth as it typically does in an exploration setting, diving waves may improve the illumination, as they may illuminate the scatterer from below when travelling back to the surface. Figure 5.14 provides an illustration.

We considered a linear velocity model with $v(z)=v_{0}+\alpha z$, setting $v_{0}=$ $1.5 \mathrm{~km} / \mathrm{s}$ and $\alpha=0.71 / \mathrm{s}$. The Green function for the 2D case is (Kuvshinov and Mulder, 2006)

$$
G\left(\omega, x_{1}, x_{2}\right)=\frac{1}{2 \pi}\left[Q_{v-1 / 2}(u)\right]^{*},
$$

with

$$
u=1+\frac{\left(x_{2}-x_{1}\right)^{2}+\left(\hat{z}_{2}-\hat{z}_{1}\right)^{2}}{2 \hat{z}_{2} \hat{z}_{1}} .
$$

$Q_{v-1 / 2}(u)$ is the Legendre function, $v=\mathrm{i} \sqrt{(\tilde{\omega} / \alpha)^{2}-1 / 4}$, and $\hat{z}_{k}=z_{k}+v_{0} / \alpha$ for $k=1,2$. Attenuation is included by letting $\tilde{\omega}=\omega[1+(i-a) /(2 Q)]$, with $a=(2 / \pi) \log \left(f / f_{r}\right)$. We have taken the complex conjugate because we adopted a Fourier convention opposite to the one used by Kuvshinov and Mulder (2006).

We chose the same acquisition and wavelet as in the first homogeneous example. Because diving waves do not reach that deep for this acquisition geometry (Mulder and Plessix, 2008), we placed the point scatterer at a depth of $250 \mathrm{~m}$. Figures 5.15 to 5.17 display the resolution function and the reconstruction of the point scatterer. There may be some numerical 

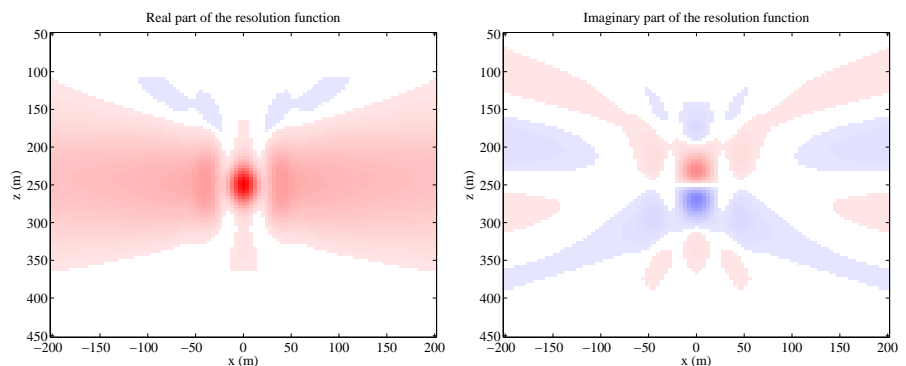

Figure 5.15: Real part (left) and imaginary part (right) of the resolution function for a point scatterer at $250 \mathrm{~m}$ depth in a linear velocity model. Colour scales are the same for both panels, with positive values in red and negative values in blue.

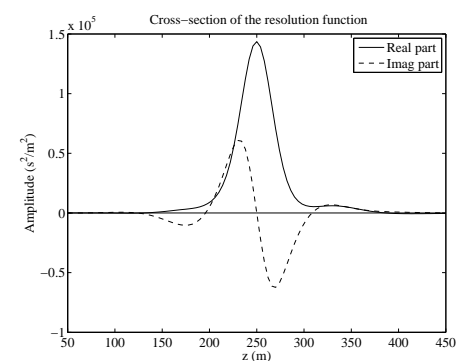

Figure 5.16: Real (drawn) and imaginary (dashed) part of the resolution function (top) along a vertical cross-section through the point scatterer at $750 \mathrm{~m}$ depth, in a linear background velocity model.
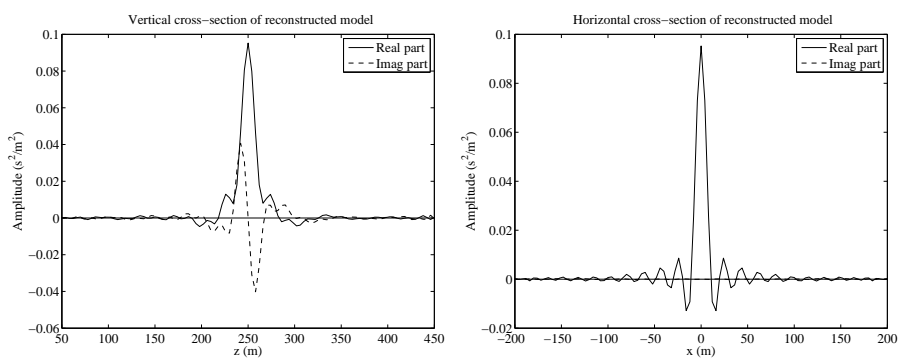

Figure 5.17: Real (drawn) and imaginary (dashed) part of the reconstructed scatterer along a vertical line (left) and horizontal line (right) through the original point scatterer position, in a linear background velocity model. 
errors due to the truncation of the Hessian to the $200 \times 200 \mathrm{~m}^{2}$ subdomain. Nevertheless, it is apparent that we have a non-negligible imaginary part in the end result. Therefore, reconstruction of both velocity and attenuation perturbation will be difficult in this setting.

\subsection{Discussion and conclusions}

We have extended the concept of the resolution function to the complexvalued case, meaning that not only impedance but also attenuation perturbations are taken into account. For the constant-density acoustic case with the Born approximation, we found that the resolution function cannot unambiguously distinguish between perturbations in velocity or in the quality factor that describes attenuation for an acquisition geometry with sources and receivers at the surface and a constant background model. This ambiguity was described in detail for horizontally layered scatterers in an earlier paper (Mulder and Hak, 2009). Here, we observed a similar behaviour for a 2D scattering model: data generated in a constant background model for the resolution function of a point scatterer were nearly identical when either the real part of this resolution function was used as a scattering model or i times its imaginary part. As a consequence, simultaneous linearised inversion for velocity and attenuation perturbation is nearly impossible.

We considered alternative acquisition geometries that potentially could reduce or remove the ambiguity. Sources and receivers on a circle surrounding the scatterer led to a unambiguous result, apart from the usual limitations caused by the finite bandwidth of the acoustic measurements. A circular acquisition is natural in, for instance, medical application, but for hydrocarbon exploration, a circular, buried acquisition is impractical and costly. We therefore examined a cross-well geometry as an alternative. Except in an exceptional case with high symmetry, the ambiguity reappears, but is not so strong as in the surface case.

Diving waves in a model that has the velocity increasing linearly with depth will improve the illumination of a shallow target compared to downand up-moving waves reflecting in a constant model. Nevertheless, this kind of illumination still provides a substantial attenuative component after migration for data that are based on a pure velocity perturbation. This leads to the conclusion that migration of seismic data to map both 
velocity and attenuation perturbations is prone to large errors.

The question remains if nonlinear inversion of seismic data will improve the situation and allow for the unambiguous determination of subsurface attenuation parameters. The work of Hicks and Pratt (2001) suggests that this may very well be the case, possibly with first carrying out the velocity updates of the model and then performing the attenuation updates (Hicks and Pratt, 2001; Rao and Wang, 2008). Another question is how the incorporation of the causality in the scatterer, which we ignored here for simplicity, will affect the linear inversion. Including causality will lead to frequency-dependent behaviour that may help to reduce or even remove the ambiguity when a sufficiently large range of frequencies is used.

\section{A Demigration for a 1D scattering model}

Equation (5.5) states that demigration of the real part of the migration image obtained from data for a real-valued point scatterer in a 2D homogeneous background model produces complex-valued frequency-domain data that are nearly the same as those resulting from demigration of $i$ times the imaginary part of that migration image. Here, we will demonstrate that a similar statement holds for a 1D layered scattering model $m(z)$ in a 3D homogeneous background. For that case, we showed earlier (Mulder and Hak, 2009) that we obtain approximately the same data for a model and its scaled, depth-weighted Hilbert transform. These data obey

$$
\mathrm{F} \mu \simeq-\mathrm{iF} \breve{\mu},
$$

with $\mu=m(z) / z$. The abbreviation $\breve{\mu}=\mathcal{H}_{z}[\mu]$ denotes the Hilbert transform of $\mu$ in depth. If $m(z)$ is localised, so non-zero for only a small range of $z$ and $z$ is not too small, the $1 / z$ scaling can be ignored. We also showed that migration produces $\mu_{0}(z)$ with the property that

$$
\mu_{0} \simeq \frac{1}{2}(\mu-i \breve{\mu}),
$$

if suitable migration weights are used. If the original $\mu$ has a zero imaginary part, which we emphasize by setting $\mu=\mu_{r}$, then $\operatorname{Re} \mu_{0} \simeq \frac{1}{2} \mu_{r}$ and $\operatorname{Im} \mu_{0} \simeq-\frac{1}{2} \breve{\mu}_{r}$. We can select the real part of $\mu_{0}$ as a new scattering model to produce data $F\left\{\operatorname{Re} \mu_{0}\right\}$. Combining equations (5.6) and (5.7), we obtain

$$
\mathrm{F}\left\{\operatorname{Re} \mu_{0}\right\} \simeq \frac{1}{2} \mathrm{~F} \mu_{r} \simeq-\frac{1}{2} \mathrm{i} \mathrm{F} \breve{\mu}_{r} \simeq \mathrm{iF}\left\{\operatorname{Im} \mu_{0}\right\} .
$$


This agrees with the more general expression in equation (5.5), found numerically for a point scatterer in a 2D homogeneous background. 


\title{
Chapter 6
}

\section{Seismic attenuation imaging with causality}

\begin{abstract}
Seismic data enable imaging of the Earth, not only of velocity and density but also of attenuation contrasts. Unfortunately, the Born approximation of the constant-density visco-acoustic wave equation, which can serve as a forward modelling operator related to seismic migration, exhibits an ambiguity when attenuation is included. Different scattering models involving velocity and attenuation perturbations may provide nearly identical data. This result was obtained earlier for scatterers that did not contain a correction term for causality. Such a term leads to dispersion when considering a range of frequencies. We demonstrate that with this term, linearised inversion or iterative migration will almost, but not fully, remove the ambiguity. We also investigate if attenuation imaging suffers from the same ambiguity when using nonlinear or full waveform inversion. A numerical experiment shows that nonlinear inversion with causality convergences to the true model, whereas without causality, a substantial difference with the true model remains even after a very large number of iterations. For both linearised and nonlinear inversion, the initial update in a gradient-based optimisation scheme that minimises the difference between modelled and observed data is still affected by the ambiguity and does not provide a
\end{abstract}


good result. This first update corresponds to a classic migration operation. In our numerical experiments, the reconstructed model started to approximate the true model only after a large number of iterations.

\subsection{Introduction}

Seismic imaging provides qualitative, structural information about the subsurface geology. Inversion - a term that we use in the mathematical sense of finding the Earth's parameters that best explain the observed data for a given type of forward modelling - leads to a quantitative description of material properties. Even with a simplified wave propagation model such as constant-density visco-acoustics, the reconstruction of not only the velocity but also the attenuation, as a function of subsurface position, can help in distinguishing between a fluid- or gas-filled rock formation.

Inversion for attenuation has been attempted by several authors with different levels of success. Ribodetti and Virieux (1998) considered linearised inversion for density, velocity, and attenuation perturbations in a given background model using ray tracing to model the wave propagation. The correction for causality was not included. They claimed successful reconstruction of the model perturbations, although their results had large errors near sharp interfaces. Hicks and Pratt (2001) considered the nonlinear inversion problem on real data and obtained convincing results with alternating updates of the velocity and the quality factor. Kamei and Pratt (2008) applied the same approach to cross-well data. Simultaneous inversion caused problems, but inverting for the velocity model first and then for the quality factor, reproduced the correct model with synthetic data. They applied their approach to real data as well. Smithyman et al. (2009) did the same on shallow reflection data. Ribodetti et al. (2007) performed nonlinear inversion in a VSP-type configuration using synthetic data. The reconstruction is not so good for simultaneous inversion of both the velocity and quality factor, but fine if the initial quality factor model is the true one. The results in these papers provide a hint at an underlying problem in the simultaneous inversion of seismic data for velocity and attenuation.

In an earlier paper (Mulder and Hak, 2009), we showed that an ambiguity occurs when performing linearised inversion with the constantdensity visco-acoustic wave equation. In linearised inversion, the Born approximation for a given background model is used. The unknowns are 
the amplitudes of the scatterers. For the constant-density visco-acoustic wave equation, scattering is due to perturbations in the velocity as well as in the quality factor that measures the attenuation. We found that two different scattering models produced nearly identical seismic surface data. As a consequence, it will be impossible to recover both velocity and attenuation parameters for scatterers in this approach.

In another study (Hak and Mulder, 2010a), we investigated if we could overcome the ambiguity by changing the acquisition geometry for collecting the seismic data. In 2D, placement of sources and receivers on a circle around a point scatterer removed the ambiguity completely, in agreement with results found by Ribodetti et al. (2000). For more common geometries, such as cross-well and surface acquisitions, the ambiguity remained. Also for a background model that had velocity increasing with depth, the ambiguity persisted, despite the fact that the target could be illuminated from above and below if it was not too deep.

In those papers (Mulder and Hak, 2009; Hak and Mulder, 2010a), we simplified our model such that our parameters were independent of frequency. Consequently, the correction term required to make a signal causal was dropped in the scattering term, although it was kept for the background model. The question remains if the inclusion of this term in the scatterer will affect the occurrence of the ambiguity. The causality term introduces a frequency dependency that translates into dispersion. Inversion over a sufficiently large band width might remove the ambiguity, as suggested by the work of Ribodetti and Hanyga (2004) and Innanen and Weglein (2007). Another question that was not addressed is if the ambiguity would persist if the Born approximation is abandoned, i.e., in nonlinear or full waveform inversion. At first sight, the problem should persist in nonlinear inversion. If a gradient-based descent algorithm is used to minimise the least-squares norm of the error between modelled and measured seismic data, the first model update in a nonlinear minimisation scheme will be identical to the one obtained for linearised inversion with the Born approximation. It can be expected that a small number of iterations will not remove the ambiguity. At some point, however, the secondary scattering effects will create a difference between the nonlinear updates and those for the linearised inversion. Although the differences will be quite small, they may, or may not, be sufficient to provide a unique answer in the nonlinear case.

Here, we address these two questions: will causal scattering in the Born approximation and will nonlinear waveform inversion, without or 
with causality, remove the ambiguity between velocity and attenuation perturbations? Section 6.2 lists the governing equations and describes the finite-difference code used for nonlinear inversion. In Section 6.3, we present results for linearised inversion for a point scatterer in a constant background model. Section 6.4 addresses nonlinear inversion for a homogeneous background model with added sombrero-shaped functions, without and with noise, and a slightly more realistic marine model. In Section 6.5, we summarise the conclusions.

\subsection{Methodology}

\subsubsection{Forward modelling}

We consider the constant-density visco-acoustic wave equation

$$
-\omega^{2} m(\omega, x) p(\omega, x)-\Delta p(\omega, x)=s(\omega, x),
$$

with $p$ the pressure wave field, $s$ a source term, $\omega=2 \pi f$ the angular frequency, $f$ the frequency, and $m$ the inverse square root of the complexvalued wave velocity obeying

$$
m(\omega, x)=\sigma^{2}(x)\left[1+\frac{\mathrm{i}-a(\omega)}{Q(x)}\right], \quad a(\omega)=\frac{2}{\pi} \log \left(\frac{\omega}{\omega_{r}}\right) .
$$

Here $Q \gg 1$ is the quality factor that describes attenuation and $\sigma$ is the slowness, both real-valued. The logarithmic term $a$ is required for causality (Aki and Richards, 2002) and is defined relative to a reference frequency $\omega_{r}=2 \pi f_{r}$, usually chosen as $f_{r}=1 \mathrm{~Hz}$.

For the Born approximation, the model is split into a smooth background component $m_{b}$, that does not produce significant reflections in the seismic frequency band and an oscillatory perturbation $\delta m$, related by $m=m_{b}+\delta m$. The scattered wavefield $\delta p$ follows from the pair of equations

$$
\begin{aligned}
& {\left[-\omega^{2} m_{b}(\omega, x)-\Delta\right] p_{b}(\omega, x)=s(\omega, x),} \\
& {\left[-\omega^{2} m_{b}(\omega, x)-\Delta\right] \delta p(\omega, x)=\omega^{2} \delta m(\omega, x) p_{b}(\omega, x) .}
\end{aligned}
$$


For a source term $s(\omega, x)=w(\omega) \delta\left(\boldsymbol{x}-\boldsymbol{x}_{\mathrm{s}}\right)$, with a wavelet $w$ and a delta function source located at $x_{\mathrm{s}}$, the solution of eq. (6.3) at a receiver location $x_{\mathrm{r}}$ can be expressed as

$$
\delta p_{\mathrm{r}(\mathrm{s})}(\omega)=w(\omega) \omega^{2} \int \mathrm{d} x G\left(\omega, x_{\mathrm{s}}, x\right) \delta m(\omega, x) G\left(\omega, \boldsymbol{x}, \boldsymbol{x}_{\mathrm{r}}\right)
$$

where the background model $m_{b}$ determines the Green function $G\left(\omega, x_{1}, x_{2}\right)$, the wavefield at $x_{2}$ due to a delta function source at $x_{1}$ or vice versa. The subscript $\mathrm{r}(\mathrm{s})$ denotes the dependency on the receiver location $x_{\mathrm{r}}$, which may itself depend on the source location $x_{\mathrm{s}}$.

In earlier papers (Mulder and Hak, 2009; Hak and Mulder, 2010a), we assumed frequency independency of the scatterer by setting $a=0$ in the scatterer, but not in the background model. In that case, eq. (6.4) can be summarised as $\mathbf{p}=\widetilde{\mathbf{F}} \delta \widetilde{\mathbf{m}}$, with a linear map $\widetilde{\mathbf{F}}$ and using scalar complex values for the scattering model $\delta \widetilde{m}(\boldsymbol{x})$ and the data $\delta p_{\mathrm{r}(\mathrm{s})}(\omega)$. The Hessian of the problem $\widetilde{\mathrm{H}}=\sum_{\omega} \widetilde{F}^{\mathrm{H}} \widetilde{F}$ was Hermitian, where the superscript $(\cdot)^{\mathrm{H}}$ denotes the conjugate transpose. Applying the Hessian to a delta-function perturbation at position $x_{\mathrm{p}}$ gave the resolution function.

If we do not ignore the causal term in the scatterer, we have to abandon the representation by complex-valued variables and treat the real and imaginary parts of the perturbation as separate model parameters. Let

$$
\begin{aligned}
& \delta m^{(1)}(x)=\sigma^{2}(x)-\sigma_{b}^{2}(x), \\
& \delta m^{(2)}(x)=\sigma^{2}(x) Q^{-1}(x)-\sigma_{b}^{2}(x) Q_{b}^{-1}(x) .
\end{aligned}
$$

Then $\delta m=\delta m^{(1)}+(\mathrm{i}-a) \delta m^{(2)}$. The inclusion of the dispersive term introduces a frequency dependency that breaks the symmetry between the real and imaginary part, which caused difficulties in our earlier papers. This might alleviate the scattering ambiguity.

In the causal case, a forward modelling operator $\mathrm{F}$ maps parameter pairs $\left\{\delta m^{(1)}, \delta m^{(2)}\right\}$ to the data:

$$
\left(\begin{array}{l}
\operatorname{Re} \mathbf{p} \\
\operatorname{Im} \mathbf{p}
\end{array}\right)=\left(\begin{array}{ll}
\mathrm{F}^{11} & \mathrm{~F}^{12} \\
\mathrm{~F}^{21} & \mathrm{~F}^{22}
\end{array}\right)\left(\begin{array}{l}
\delta \mathbf{m}^{(1)} \\
\delta \mathbf{m}^{(2)}
\end{array}\right)
$$


This can be related to the earlier, simplified choice $\widetilde{\mathrm{F}}$ with $a=0$ by

$$
\begin{array}{ll}
\mathrm{F}^{11}=\operatorname{Re} \widetilde{F}, & F^{12}=-\operatorname{Im} \widetilde{F}-a \operatorname{Re} \widetilde{F}, \\
F^{21}=\operatorname{Im} \widetilde{F}, & F^{22}=\operatorname{Re} \widetilde{F}-a \operatorname{Im} \widetilde{F} .
\end{array}
$$

Note that now all values are real. The corresponding Hessian becomes

$$
H=\sum_{\omega} F^{\top} F=\left(\begin{array}{ll}
H^{11} & H^{12} \\
H^{21} & H^{22}
\end{array}\right) .
$$

The superscript $(\cdot)^{\top}$ denotes the transpose.

By applying this Hessian to a model with a delta-function perturbation at position $x_{\mathrm{p}}$, we obtain four resolution functions now, one for each operator $\mathrm{H}^{i j}$. For $j=1$, we obtain the pair for a delta-function perturbation of $\delta m^{(1)}$, producing $g^{11}$ and $g^{21}$, the images in the first and second component, respectively. Similarly, we find the pair for a delta function perturbation of the second model component, $\delta m^{(2)}$, for $j=2$, producing images $g^{12}$ and $g^{22}$. The first pair corresponds mainly to a velocity perturbation and the second pair corresponds to an attenuation perturbation. Ideally, $g^{11}$ and $g^{22}$ should resemble spikes at the position of the scatterer whereas $g^{21}$ and $g^{12}$ should be small.

\subsubsection{Linearised inversion}

Least-squares inversion for the scatterer in a given background model that is kept fixed amounts to solving the linear problem

$$
\mathrm{H} \delta \mathbf{m}=\mathrm{H} \delta \widehat{\mathbf{m}},
$$

where $\delta \widehat{\mathbf{m}}$ is the true scatterer and $\delta \mathbf{m}$ its reconstruction. As the Hessian $\mathrm{H}$ is singular, the linear system can be solved by means of the pseudo-inverse $\mathrm{H}^{\dagger}$. This provides the minimum-norm solution. The system may also be solved by an iterative method that can handle its null-space, for instance, the conjugate-gradient method. Without preconditioning, this method should also provide the minimum-norm solution. With preconditioning, the usual norm is replaced by a weighted norm, determined by the preconditioner, and the solution of the singular problem will differ from the one obtained without preconditioning. 


\subsubsection{Nonlinear inversion}

We used a frequency-domain finite-difference code (Mulder and Plessix, 2004) to compute synthetic data and carry out the least-squares inversion. As pointed out by Marfurt and Shin (1989), the solution of eq. (6.1) for $2 \mathrm{D}$ problems is more efficient in the frequency domain than in the time domain. The discretisation of eq. (6.1) leads to a large but sparse matrix. Its LU-decomposition can be computed by nested dissection (George and Liu, 1981). This step is costly, but is required only once for each frequency. The result can then be used for all shots and also for back-propagation during migration and inversion. The spatial discretisation is a compact fourthorder finite-difference scheme with a 9-point stencil. We used the generic fourth-order choice (Harari and Turkel, 1995) rather than the parameters suggested by Jo et al. (1996). In their notation, our choice amounts to $a=c=2 / 3, d=1 / 12$, and $e=1-c-4 d=0$.

Nonlinear inversion of the least-squares functional that measures the misfit between modelled and observed data was carried out by the LBFGS method (Byrd et al., 1995). The unknowns where parametrised by $\sigma^{2}$ and $\sigma^{2} Q^{-1}$, depending on subsurface position. The required gradient of the functional with respect to the model parameters was derived by the adjoint-state method, applied to the discretised equations. Details are given in Appendix 6.A. For regularisation purposes, we added a penalty term to the least-squares error functional that is a measure of the differences between the current estimated model and the given initial background model. The relative weight of this term was made very small.

\subsection{Linearised inversion in constant background}

We start with the same example as in our earlier paper (Hak and Mulder, 2010a): a point scatterer in a 2D constant background model with velocity $c_{b}=2000 \mathrm{~m} / \mathrm{s}$ and quality factor $Q_{b}=100$. The scatterer is located at $x=0$ and $z=750 \mathrm{~m}$. Sources are placed between -1887.5 and $+1887.5 \mathrm{~m}$ at a $25-$ $\mathrm{m}$ interval and receivers between -1900 and $+1900 \mathrm{~m}$ at the same interval, all at zero depth. We computed the Hessian on a grid with $101 \times 101$ points and a spacing of $4 \mathrm{~m}$, having the scatterer at the centre. This resulted in a Hessian matrix of size $20402 \times 20402$. We used a Ricker wavelet with a peak frequency of $15 \mathrm{~Hz}$ and considered frequencies in the range between 
4 and $20 \mathrm{~Hz}$ at a $0.5-\mathrm{Hz}$ interval.

Fig. 6.1 shows the resolution functions, all on the same colour scale, with red for positive and blue for negative values. A delta-function perturbation of the first component produces a band-limited version, $g^{11}$, after migrating the data, but also produces a substantial contribution in
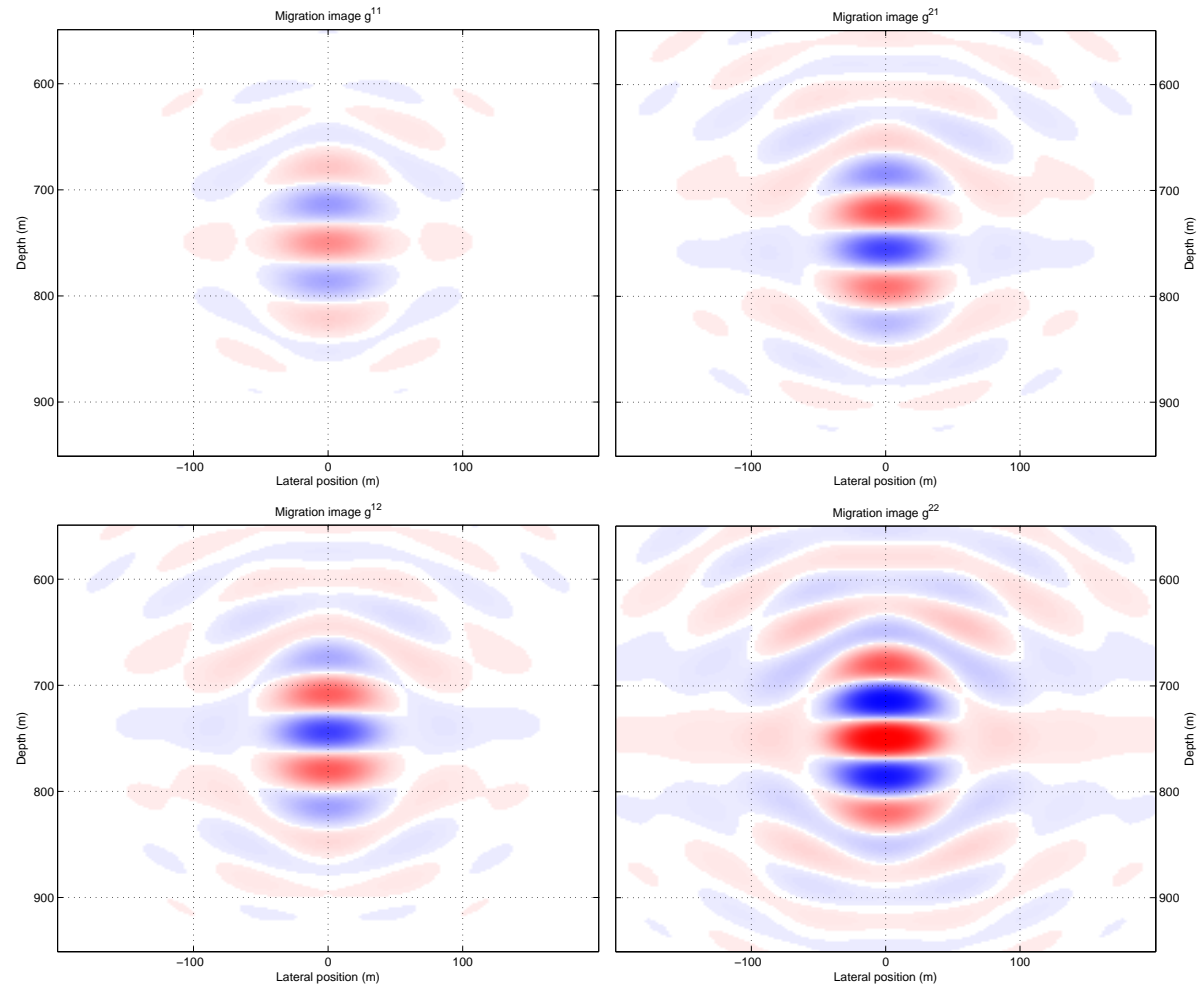

Figure 6.1: Resolution functions for a delta-function perturbation of the first model parameter (top row) and the second model parameter (bottom row), all plotted with the same colour scale. Ideally, the pictures on the diagonal should resemble a band-limited version of a spike at the point scatterer, as they do, and the off-diagonal pictures should have much smaller amplitudes, which they do not. Note that $g^{21}$ differs from $g^{12}$. 


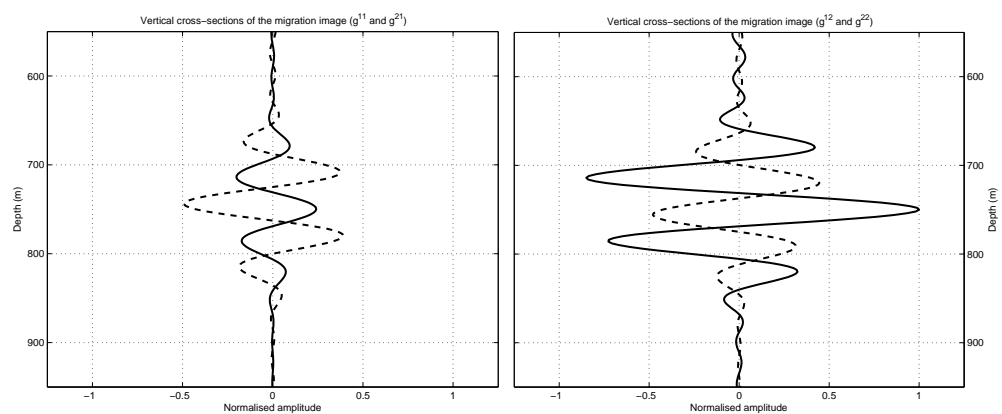

Figure 6.2: Vertical cross-sections of the resolution function through the scattering point. The left panel corresponds to a delta-function perturbation of the first component, the right one to a perturbation of the second component. The solid line represents the perturbed component, the dashed line is its contribution in the unperturbed component ( $g^{21}$ and $g^{12}$ for the left and right panel, respectively). There is a significant contribution (crosstalk) in the unperturbed component, similar to the case of a scatterer without a causal term. The shape of the crosstalk, however, is rather different from the one obtained without the causal term, where there is a $90^{\circ}$ phase difference. Here, the crosstalk has almost the opposite phase.

the other component, $g^{21}$, with an even larger amplitude. Similar crosstalk occurs for a delta-function perturbation of the second component. Its migration image, $g^{22}$, looks reasonable, but there is substantial contribution to $g^{12}$, although with a smaller amplitude than $g^{21}$. Fig. 6.2 displays vertical cross-sections through the scatterer point of the resolution functions for a delta-function perturbation of the first (left panel) and second component (right panel). Compared to the case of a scatterer with causal correction term, presented elsewhere (Hak and Mulder, 2010a), we note that the crosstalk into the wrong component is still substantial, but now has a phase difference of almost $180^{\circ}$ instead of $90^{\circ}$.

Because the crosstalk is still large, simultaneous imaging of velocity and attenuation perturbations will lead to erroneous results if just a single step is carried out. However, if we try to solve eq. (6.9) by iterative migration, the result may improve. In the current example, we directly computed the pseudo-inverse of the Hessian and obtained the reconstructions shown in Fig. 6.3. The left panel displays a vertical cross-section obtained for a delta-function perturbation of the first component, the right panel for a perturbation of the second. The inversion has considerably reduced the 

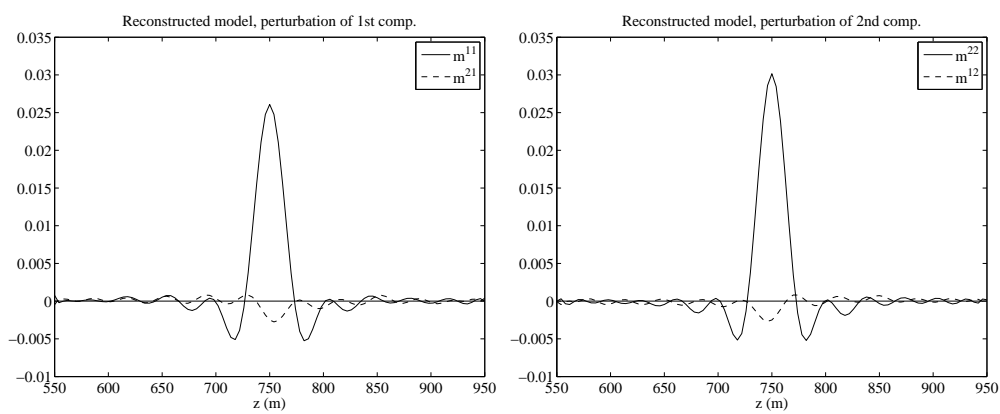

Figure 6.3: Vertical cross-sections through the scattering point of the reconstructed perturbation. The left panel corresponds to a delta-function perturbation of the first component, the right one to a perturbation of the second component. The solid line represents the reconstruction of the perturbed component, the dashed line is its contribution (crosstalk) in the unperturbed component $\left(\mathrm{m}^{21}\right.$ and $\mathrm{m}^{12}$ for the left and right panel, respectively). In both cases, a band-limited reconstruction is provided. A remnant in the unperturbed component is still present, but is considerably smaller than in the case of a scatterer without a causal term.

amount of crosstalk and provided an acceptable band-limited reconstruction of the scatterer.

The results show that inclusion of the frequency-dependent causal correction term in the scatterer provides sufficient additional information to enable a better reconstruction. This agrees with the work of Ribodetti and Hanyga (2004) and Innanen and Weglein (2007). However, in a iterative migration approach, the substantial crosstalk in the first iteration suggests that a large number of iterations will be required before the crosstalk is reduced to an acceptable level.

Indeed, we found that the solution of eq. (6.9) by a conjugate-gradient method without preconditioning took of the order of $10^{4}$ iterations to obtain the same result as in Fig. 6.3. More precisely, 11, 445 iterations were required for a decrease of the residual by a factor $10^{-7}$. With preconditioning by a diagonal matrix with 1 for the first unknown, $m^{(1)}$, and $Q_{b}$ for the second unknown, $m^{(2)}$, at each grid point the number was reduced to 8016. This is still substantial and perhaps too large to be of practical use in applications of iterative migration (Østmo et al., 2002; Mulder and Plessix, 2004). 

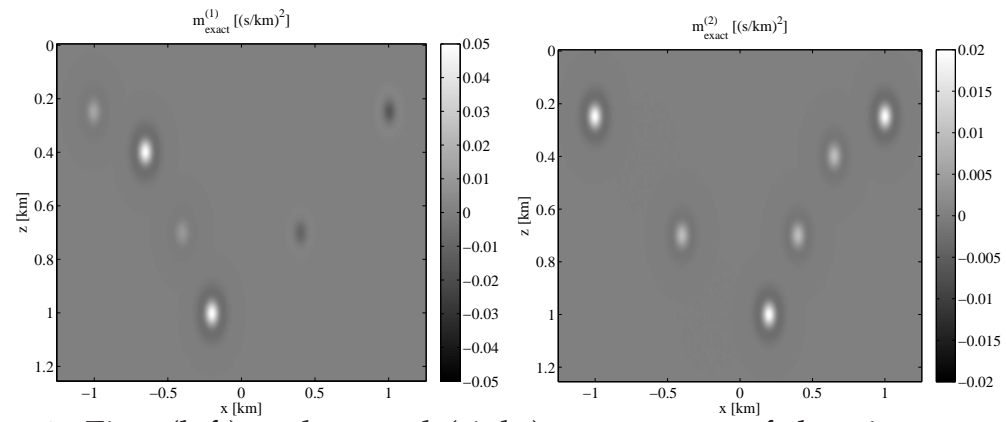

Figure 6.4: First (left) and second (right) component of the given model after subtraction of the background model.

\subsection{Nonlinear inversion}

\subsubsection{Constant velocity model}

Given the severe limitations of the Born approximation, nonlinear inversion is more natural. We take a homogeneous background model with a velocity $c_{b}=1500 \mathrm{~m} / \mathrm{s}$ and quality factor $Q_{b}=100$ and add a number of sombrero-shaped functions to it. We take this sombrero function as minus the Laplace operator applied to a Gaussian, or

$$
(1-\eta) \exp (-\eta) \text {, with } \eta=\frac{\left(x-x_{0}\right)^{2}+\left(z-z_{0}\right)^{2}}{2 \sigma_{g}^{2}},
$$

which in 1D would have the shape of a Ricker wavelet. Fig. 6.4 shows $\delta m^{(1)}$ and $\delta m^{(2)}$ as defined in eq. (6.5), the difference between the model and the background represented by the squared slowness and the squared slowness divided by the quality factor, respectively. For the sombrero function, we chose a standard deviation $\sigma_{g}$ of $40 \mathrm{~m}$ and used various amplitudes. The peak value for the first component, shown in the left panel of Fig. 6.4, was $510^{-8}(\mathrm{~s} / \mathrm{m})^{2}$. The second component, shown on the right, had a peak value of $210^{-8}(\mathrm{~s} / \mathrm{m})^{2}$. The amplitudes of the sombrero functions for each component can be deduced from the vertical crosssections in the figures to follow.

We generated synthetic data for the constant-density visco-acoustic wave equation with the frequency-domain finite-difference code mentio- 
ned earlier, without and with the correction term for causality. Absorbing boundary conditions were imposed on all four sides of the computational domain. We used the same acquisition geometry and wavelet as in the earlier example. A different computational grid was used with a 10-m grid spacing on a domain with $x$ between -2500 and $2500 \mathrm{~m}$ and $z$ between -500 and $1500 \mathrm{~m}$. The model parameters were kept fixed for $z<50 \mathrm{~m}$.

Fig. 6.5 shows the reconstructed model after 29,924 iterations, where the causal correction term was applied in the generation of the original data and in the inversion. The least-squares functional $\mathcal{J}$, given in eq. (6.11), decreased from its initial value by a factor $10^{-7}$. Vertical cross-sections are displayed in Fig. 6.6. The figure shows multiple curves, each one corresponding to a vertical line through the centre of one of the sombrero functions. The dashed lines correspond to the true model perturbations. There are separate graphs for the the first and second component of the model perturbations as well as the cross-sections at negative and positive $x$. From the relative positions of the peaks, the reader should be able to find the corresponding scatterer. The shallower part of model is reconstructed accurately, whereas the deeper parts have not converged to the true model.

We repeated this exercise without applying the causal correction in the generation of the original data nor in the inversion. The least-squares function $\mathcal{J}$ decreased from its initial value by a factor $10^{-7}$ after 59,904 iterations. Fig. 6.7 shows the reconstructed model. Vertical cross-sections are displayed in Fig. 6.8. Compared to the causal case, the deviations from the true model remain substantial even after so many iterations. This suggests that the nonlinearity by itself does not fully remove the ambiguity between the first and second component of the model parameter, at least not when the number of iterations is limited, as will happen in practical applications.

\subsubsection{Noise}

We performed the same numerical experiment for the causal case with noise added to the data. The earlier frequency domain data were transformed to the time domain for a Ricker wavelet with a peak frequency of $15 \mathrm{~Hz}$. We took the maximum amplitude of the first shot, multiplied it by $10^{-4}$ and added random numbers between -1 and +1 times this amplitude to all the data. The result was then transformed back to the frequency 

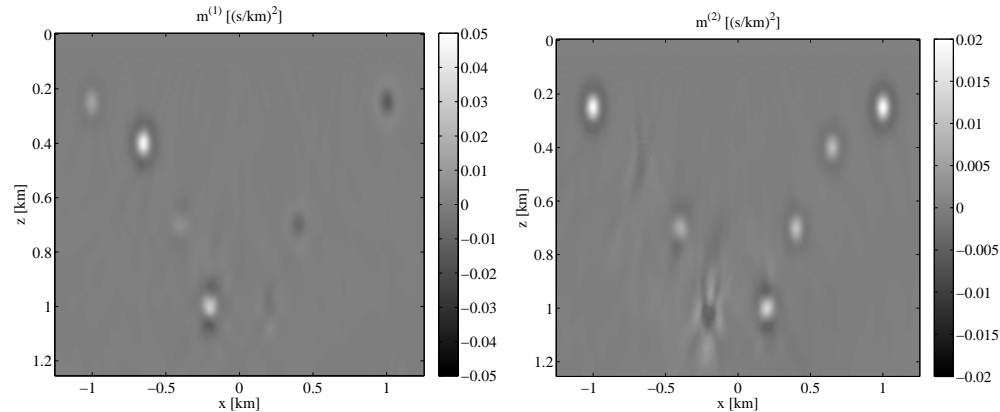

Figure 6.5: First (left) and second (right) component of the reconstructed perturbation model after about 30,000 iterations.
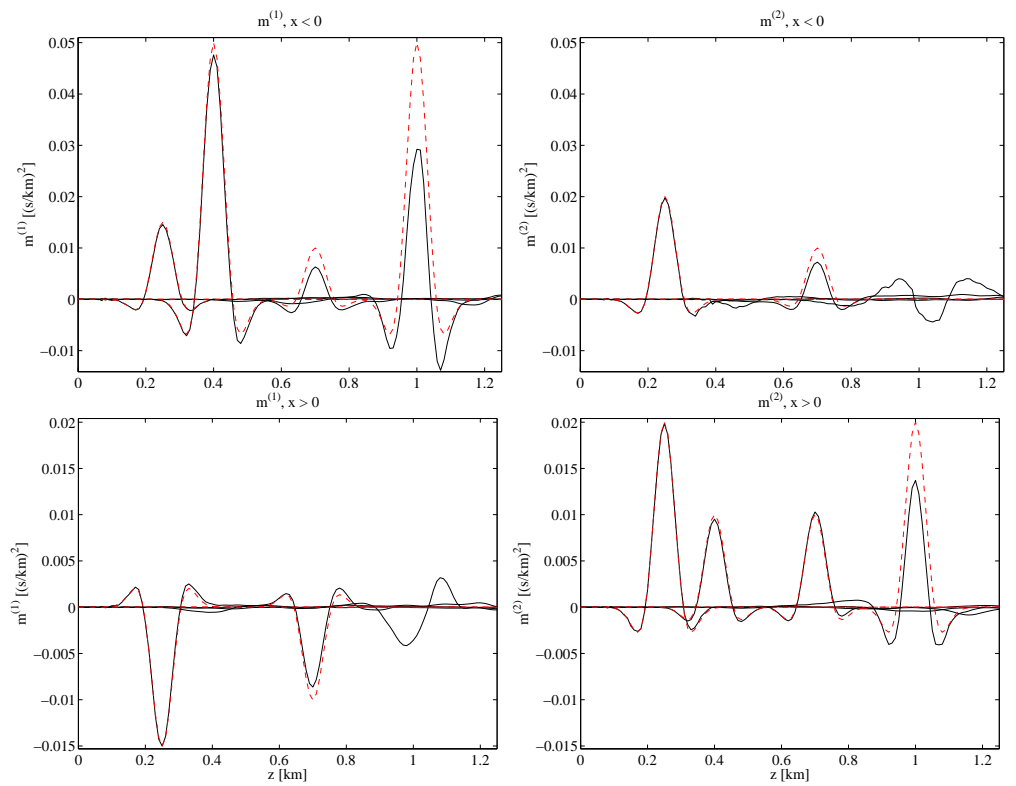

Figure 6.6: Vertical cross-sections of the reconstructed model after about 30,000 iterations. The left panels show the first component, those on the right the second component. The top row contains sections for $x=-1000,-650,-400$, and $-200 \mathrm{~m}$ and the bottom for $x=200,400,650$, and $1000 \mathrm{~m}$. The solid lines represent the reconstructed model perturbations, the true model perturbations are drawn as red dashed lines. 

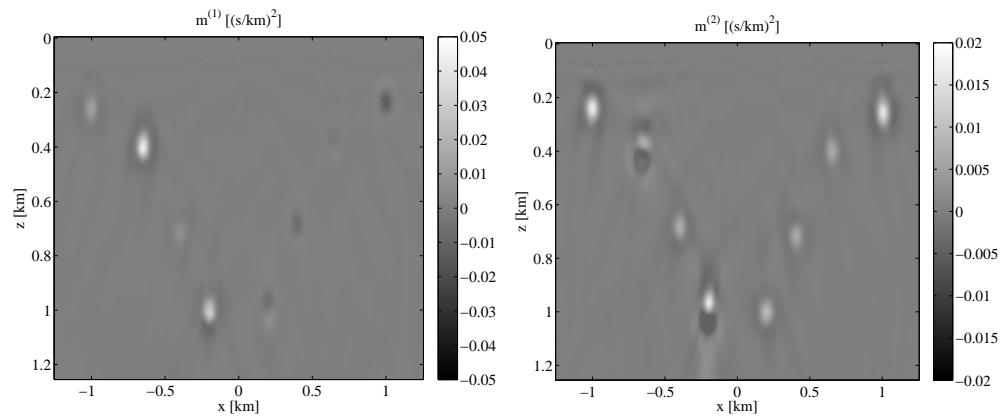

Figure 6.7: First (left) and second (right) component of the reconstructed perturbation model after about 60,000 iterations. The correction for causality was not applied in the data generation nor the inversion.
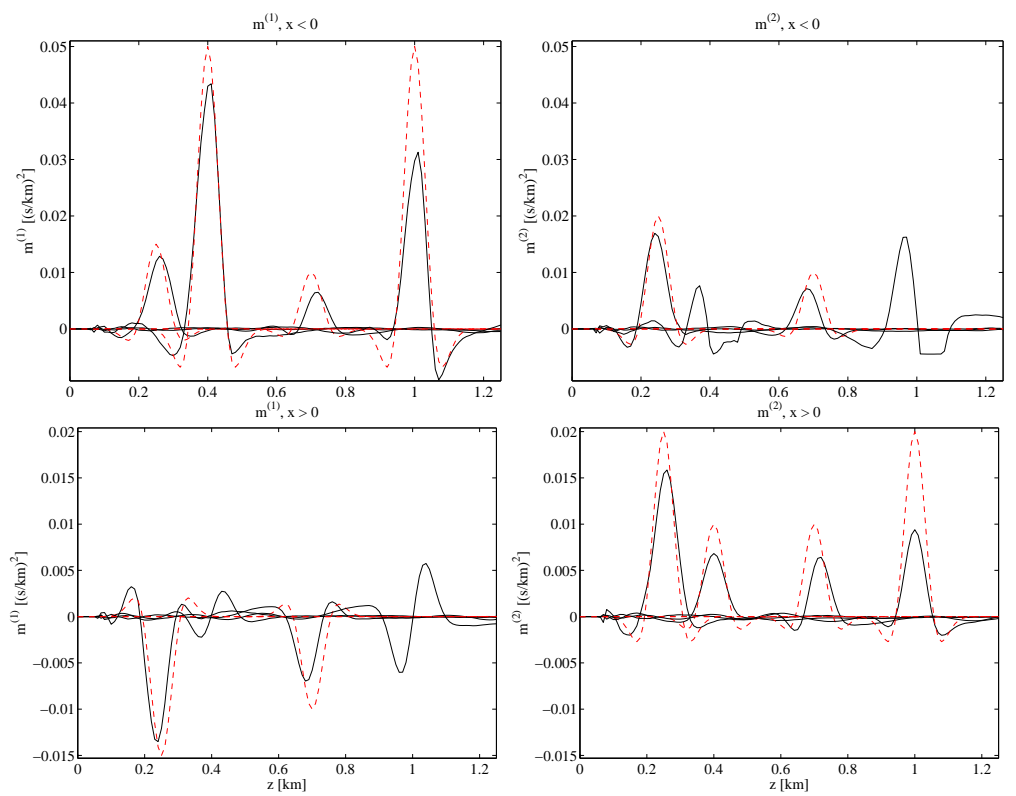

Figure 6.8: As Fig. 6.6, but without applying the corrections for causality in the data generation nor the inversion and after about 60,000 iterations. 
domain.

Fig. 6.9 shows vertical cross-sections of the reconstructed model after about 5,000 iterations. The result is qualitatively correct but the amplitude discrepancies still are substantial. The result after about 30,000 iterations, shown in Fig. 6.10, is clearly better, but the computational cost is huge. Also, the noise level is higher. This might be improved by adding smoothing penalty terms to the least-squares functional. Compared to the acausal case, the reconstruction result appears to be better.
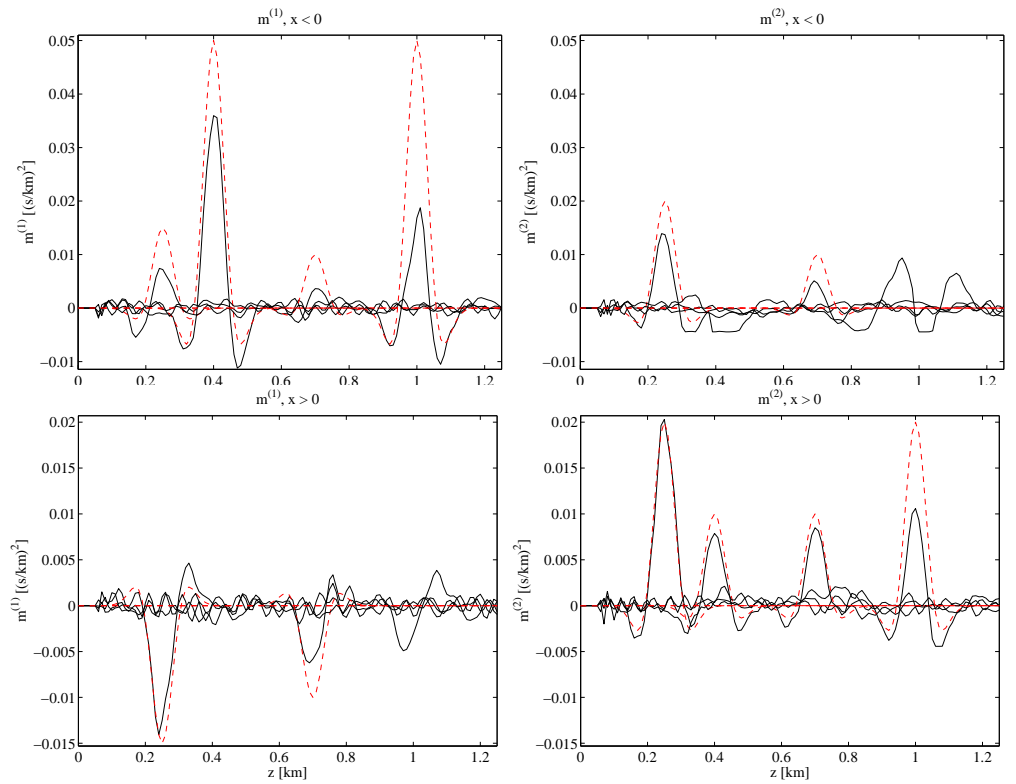

Figure 6.9: As Fig. 6.6, but for data with a relative noise level of $10^{-4}$ and after about 5,000 iterations. 

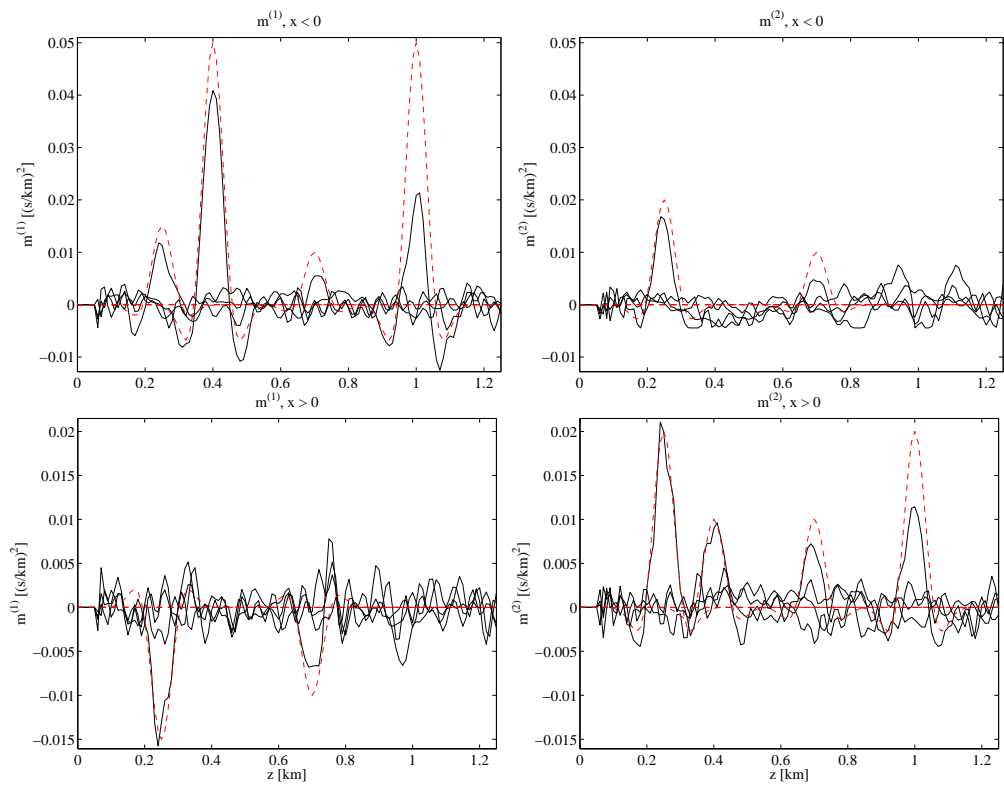

Figure 6.10: As Fig. 6.9, but after about 30,000 iterations. The reconstruction of the scatterers has improved, but the noise level in the image has increased as well.

\subsubsection{Marine model}

Fig. 6.11 shows a geophysically slightly more realistic model than used so far. Below the sea bottom, the velocity is horizontally layered. A rectangular region with low quality factor, as may happen with shallow gas, is included. Fig. 6.12 displays a vertical cross-section of a smoothed version of the model without the strongly attenuating rectangular region, which served as an initial guess for the nonlinear inversion. For our computations, we used a grid spacing of $10 \mathrm{~m}$. Causality was included.

Fig. 6.13 shows the result of the reconstruction after 10,015 iterations, when the least-squares cost function had decreased by a factor $1.110^{-5}$ from its initial value. The velocity still shows an imprint of the rectangular region. Fig. 6.14 displays vertical cross-sections through centre.

We continued the iterations to a total of 49,363 and a corresponding decrease of the least-squares cost function by a factor $9.310^{-7}$. Figs 6.15 and 6.16 show an improvement over the earlier result. The imprint of the 

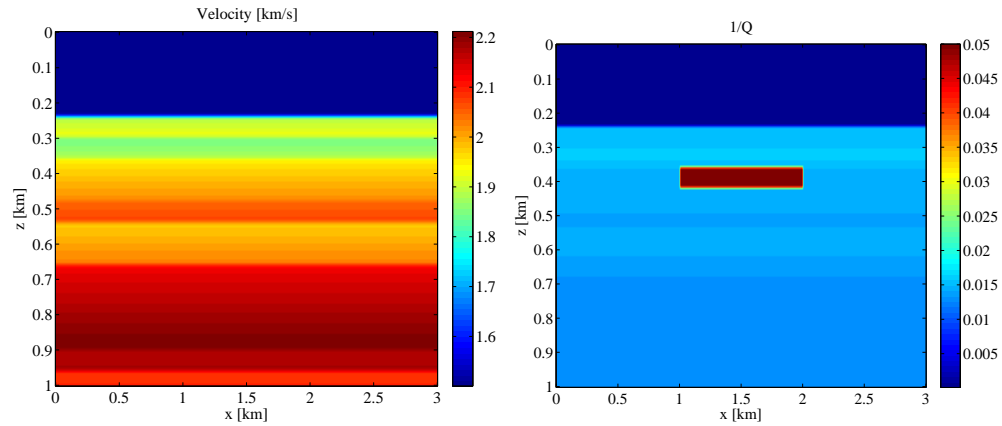

Figure 6.11: Velocity (left) and inverse quality factor (right) of the given model.
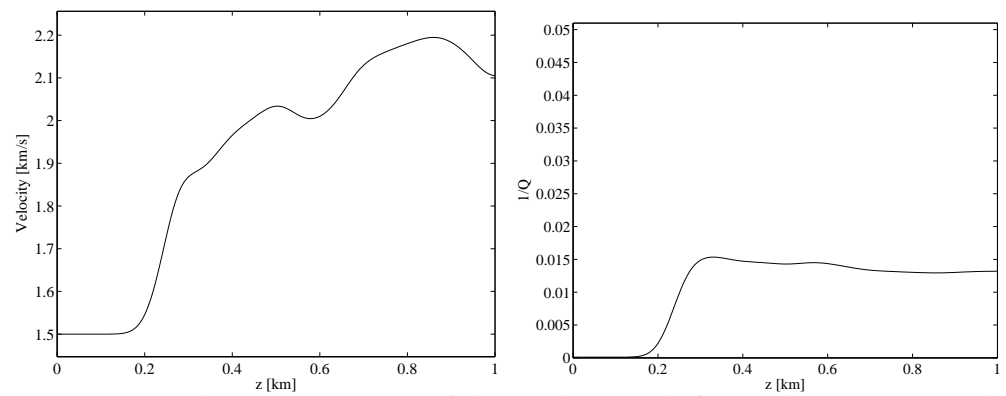

Figure 6.12: Vertical cross-section of the velocity (left) and inverse quality factor (right) of the initial model.
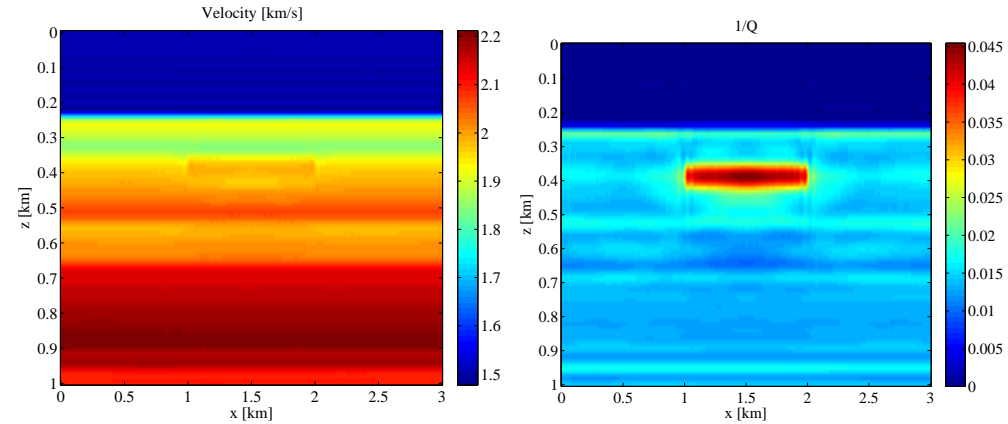

Figure 6.13: Velocity (left) and inverse quality factor (right) of the reconstructed model after about 10,000 iterations. 

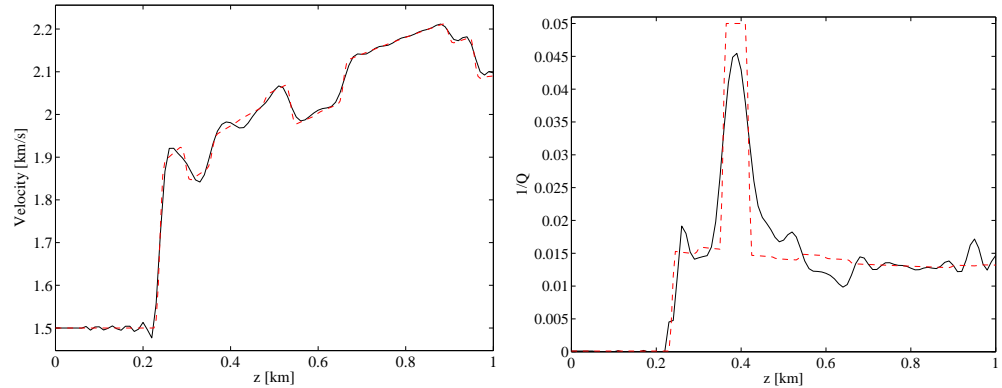

Figure 6.14: Vertical cross-section at $x=1.5 \mathrm{~km}$ of the reconstructed velocity (left) and inverse quality factor (right) after about 10,000 iterations. The solid line represents the reconstructed model, the true model is drawn as red dashed line.
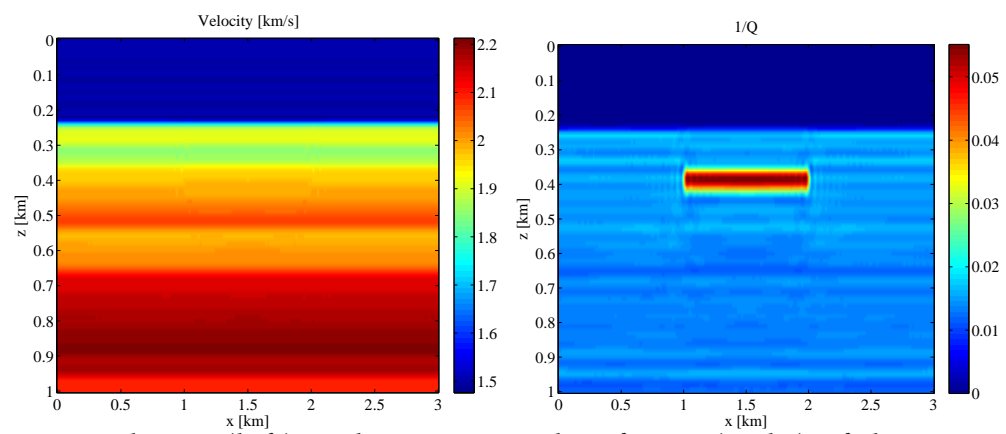

Figure 6.15: Velocity (left) and inverse quality factor (right) of the reconstructed model after about 50,000 iterations.
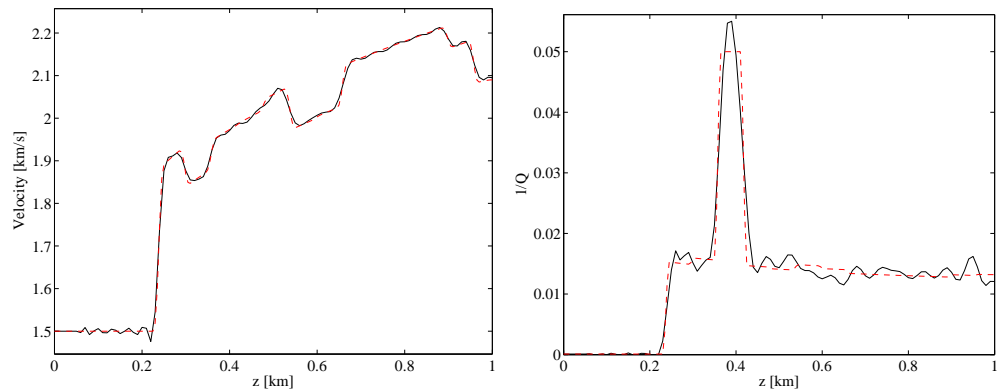

Figure 6.16: As Figure 6.14, but after about 50,000 iterations. 
strongly attenuating region on the reconstructed velocity model has almost disappeared and the quality factor is closer to the true model. We conclude that the inversion produces the correct result, but at a discouragingly large cost.

\subsection{Conclusions}

We have investigated how two methodologies are affected by an ambiguity that occurs for attenuation scattering imaging based on the Born approximation. The first one is the inclusion of the correction for causality in the scatterer. This introduces a frequency-dependent dispersion term. When using a range of frequencies, this term almost, but not completely, removes the ambiguity that otherwise occurs between the real and imaginary parts of the scattering model.

Because the Born approximation offers no handle on the energy of the scattered data and is invalid for larger scattering angles when, for instance, flat scatterers are considered, a nonlinear approach is more natural and we took this as a second methodology. In an example, we observed that nonlinearity is not able to completely remove the ambiguity if the causal correction term is dropped, at least not for a large but limited number of iterations. With the causal term, the reconstruction succeeds.

In both cases, the initial update of a gradient-based optimisation algorithm that tries to minimise the difference between modelled and observed data differs significantly from the correct update. Many iterations are therefore required to approach the true solution. We observed the same when adding noise and for a slightly more realistic example, both with causality included. This implies that, although causality enabled us to resolve the ambiguity in attenuation imaging in the examples considered here, it will be difficult to actually find the true model in realistic cases when noise is present and the number of iterations has to be limited because of computational cost.

\section{A Gradient of the least-squares functional}

The gradient of the least-squares functional that measures the difference between synthetic and observed data can be derived by the adjoint-state 
method. The derivation for complex parameters, though straightforward, is somewhat less obvious than that for real-valued parameters, listed for instance by Plessix (2006), so we present it below.

In the frequency domain, the least-squares error functional for the nonlinear problem is

$$
\begin{aligned}
& \mathcal{J}=\frac{1}{2} \sum_{\omega, s, \mathrm{r}(\mathrm{s})}\left|\varepsilon_{\mathrm{r}(\mathrm{s})}(\omega)\right|^{2}, \\
& \varepsilon_{\mathrm{r}(\mathrm{s})}(\omega)=w(\omega) \int \mathrm{d} x \mathrm{R}_{\mathrm{r}(\mathrm{s})} \bar{p}(\omega, x)-p_{\mathrm{r}(\mathrm{s})}^{\mathrm{obs}}(\omega),
\end{aligned}
$$

with $p_{\mathrm{r}(\mathrm{s})}^{\mathrm{obs}}$ the observed data and $\bar{p}$ the modelled wavefield obtained for a temporal delta function at zero time and a spatial delta source at $x_{\mathrm{s}}$. Here $\mathrm{s}$ indexes shot positions and $\mathrm{r}(\mathrm{s})$ indexes receivers positions for shot $\mathrm{s}$. We have included a wavelet $w$ and a real-valued sampling operator $\mathbf{R}$ that is a restriction of the modelled wavefield to the receiver location. If the wavelet is the same for all shots, minimisation of $\mathcal{J}$ with respect to the wavelet leads to

$$
w(\omega)=\frac{\sum_{\mathrm{s}, \mathrm{r}(\mathrm{s})}\left[\int \mathrm{d} x \mathrm{R}_{\mathrm{r}(\mathrm{s})} \bar{p}(\omega, x)\right]^{*} p_{\mathrm{r}(\mathrm{s})}^{\mathrm{obs}}(\omega)}{\sum_{\mathrm{s}, \mathrm{r}(\mathrm{s})}\left|\int \mathrm{d} x \mathrm{R}_{\mathrm{r}(\mathrm{s})} \bar{p}(\omega, x)\right|^{2}} .
$$

This step can be skipped if the wavelet is known and specified in advance.

To determine the gradient of $\mathcal{J}$ with respect to the model parameters, we can use the adjoint-state method. For brevity, we only consider a single shot at a single frequency such that we can drop the indexing subscripts and writing dependency on the frequency. The gradients for all frequencies and shots can be obtained by summation in the end.

Let the wave equation for a temporal delta function at zero time and a spatial delta source at $x_{s}$ be given by $\mathrm{P}=\mathrm{L} \bar{p}-\delta\left(x-x_{s}\right)=0$, where the operator $L=-\omega^{2} m-\Delta$. We choose a Lagrangian

$$
\mathcal{L}=\mathcal{J}-\int \mathrm{d} x q_{r}(x) \mathrm{P}_{r}(x)-\int \mathrm{d} x q_{i}(x) \mathrm{P}_{i}(x),
$$


where the subscripts $r$ and $i$ denote the real and imaginary parts, respectively, and $q_{r}$ and $q_{i}$ are the multipliers. Let $m=m_{r}+\mathrm{i} m_{i}$, such that $\mathrm{L}=\mathrm{L}_{r}+\mathrm{i} \mathrm{L}_{i}$. Stationarity of $\mathcal{L}$ with respect to $\bar{p}=\bar{p}_{r}+\mathrm{i} \bar{p}_{i}$ leads to

$$
\begin{aligned}
& \frac{\partial \mathcal{J}}{\partial \bar{p}_{r}}=\operatorname{Re}\left[\sum_{\mathrm{r}(\mathrm{s})} w^{*} \mathrm{R}^{\top} \varepsilon\right]-\left(\mathrm{L}_{r}^{\top} q_{r}+\mathrm{L}_{i}^{\top} q_{i}\right)=0, \\
& \frac{\partial \mathcal{J}}{\partial \bar{p}_{i}}=\operatorname{Im}\left[\sum_{\mathrm{r}(\mathrm{s})} w^{*} \mathrm{R}^{\top} \varepsilon\right]-\left(\mathrm{L}_{r}^{\top} q_{i}-\mathrm{L}_{i}^{\top} q_{r}\right)=0,
\end{aligned}
$$

which, defining $q=q_{r}+\mathrm{i} q_{i}$, can be summarised as

$$
\mathrm{L}^{\mathrm{H}} q=\sum_{\mathrm{r}(\mathrm{s})} w^{*} \mathrm{R}^{\top} \varepsilon .
$$

Let $g_{r}=\partial \mathcal{J} / \partial m_{r}$ and $g_{i}=\partial \mathcal{J} / \partial m_{i}$ be the gradient of the least-squares functional with respect to the real and imaginary part of $m$, respectively. Then

$$
\begin{aligned}
& g_{r}=\left(\mathrm{L}_{r r} p_{r}\right) q_{r}-\left(\mathrm{L}_{i r} p_{i}\right) q_{r}+\left(\mathrm{L}_{r r} p_{i}\right) q_{i}+\left(\mathrm{L}_{i r} p_{r}\right) q_{i} \\
& g_{i}=-\left(\mathrm{L}_{i r} p_{r}\right) q_{r}-\left(\mathrm{L}_{r r} p_{i}\right) q_{r}-\left(\mathrm{L}_{i r} p_{i}\right) q_{i}+\left(\mathrm{L}_{r r} p_{r}\right) q_{i}
\end{aligned}
$$

where $\mathrm{L}_{r r}=\partial \mathrm{L}_{r} / \partial m_{r}$ and $\mathrm{L}_{i r}=\partial \mathrm{L}_{i} / \partial m_{r}$, and the Cauchy-Riemann relations imply that $\mathrm{L}_{r r}=\mathrm{L}_{i i}=\partial \mathrm{L}_{i} / \partial m_{i}$ and $\mathrm{L}_{i r}=-\mathrm{L}_{r i}=-\partial \mathrm{L}_{r} / \partial m_{i}$. Using $g=g_{r}+\mathrm{i} g_{i}$ the above can be summarised as

$$
g=\left(\frac{\partial \mathrm{L}}{\partial m_{r}} p\right)^{*} q=\left(\omega^{2} p\right)^{*} q .
$$

This includes cases with complex $\omega$.

When the correction term for causality is non-zero, we need another step. For $a=(2 / \pi) \log \left(\omega / \omega_{r}\right)$, we have $m=\sigma^{2}[1+(\mathrm{i}-a) / Q]$. If we choose $m^{(1)}=\sigma^{2}$ and $m^{(2)}=\sigma^{2} Q^{-1}$ as unknowns, the corresponding gradient can be computed with the chain rule, resulting in

$$
\left(\begin{array}{l}
\frac{\partial \mathcal{J}}{\partial m^{(1)}} \\
\frac{\partial \mathcal{J}}{\partial m^{(2)}}
\end{array}\right)=\left(\begin{array}{rr}
1 & 0 \\
-a & 1
\end{array}\right)\left(\begin{array}{l}
g_{r} \\
g_{i}
\end{array}\right) .
$$


A similar approach can be taken for the Born approximation in eq. (6.3). The wave equation can be chosen as $\delta \mathrm{P}=\mathrm{L}_{b} \delta p-\omega^{2} \delta m p_{b}=0$ and the corresponding Lagrangian may be written as

$$
\mathcal{L}(x)=\mathcal{J}(x)-\int \mathrm{d} x q_{r}(x) \delta \mathrm{P}_{r}(x)-\int \mathrm{d} x q_{i}(x) \delta \mathrm{P}_{i}(x) .
$$

The adjoint equation is similar to eq. (6.16), but now the right-hand side is based on primaries and the operator is the one for the background, $L_{b}$, applying to the background model only. The gradient with respect to $\delta m$ is similar to the one before, $g=\left(\omega^{2} p_{b}\right)^{*} q$, and the correction for the causal term of eq. (6.19) can be applied if necessary.

The Hessian for the nonlinear inversion problem becomes the same as the Hessian for the Born approximation if the Gauss-Newton approximation is used, that is, if we assume that we are sufficiently close to the true model that the data differences, $\varepsilon$, and related expressions, such as $q$ in eq. (6.16), can be neglected. 


\section{Chapter 7}

\section{Conclusions and further research}

\subsection{Conclusions}

We investigated migration of recorded data for the constant-density viscoacoustic wave equation with the goal to accurately find scatterer locations and true amplitudes. We have seen that reflections in a visco-acoustic medium are due to complex-valued impedance contrasts. For a constant density medium that means they are due to spatial changes in the wave velocity and/or the quality factor of the medium.

We have investigated different perturbations, causal and non-causal. simplicity. In Chapters 3-5, a non-causal model was used for computational simplicity. Being unsuccessful in retrieving the true scatterer location and amplitude, we investigated the influence of the causality term in Chapter 6. We considered different acquisition geometries and models, including a circular setup and a cross-well setting, and a model that permits diving waves. We investigated both the linear and non-linear equations, with and without the causality term, and linear equations with and without noise. Most work was done on point scatterers, but Chapters 4 and 6 also include a more realistic model, though still synthetic.

In Chapter 3, we investigated with a iterative migration scheme, if the preconditioner can help us to retrieve simultaneous velocity and attenua- 
tion perturbations. We drew two conclusions, namely:

- There is a fundamental problem in getting reliable true-amplitude migration results for the constant density visco-acoustic wave equation.

- It is not possible to simultaneously find a reliable velocity and attenuation estimate with an iterative migration scheme, where different preconditioners may lead to different results.

In Chapter 4, we try to better understand the fundamental problem we saw in Chapter 3. Here, we found that:

- Iterative migration or linearised inversion of seismic visco-acoustic data comprises an ambiguity that goes beyond the ill-posedness caused by the data being band-limited and the acquisition being bounded.

- For visco-acoustics, different models can produce nearly identical receiver data for a horizontally layered medium.

- The migration image of a point-scatterer perturb in only the real part is nearly identical to the migration image of a point-scatterer perturbed in only the imaginary part, multiplied by a factor $-i$.

- For a horizontally layered medium, the reconstructed model may be any model that, after applying a depth-weighting, is the weighted sum of the true depth-weighted model and its Hilbert transform in the vertical coordinate multiplied with a factor $-\mathrm{i}$.

- We noticed, but not prove, that a scattering model that equals the real part of the resolution function produces nearly the same data as a model that equals i times the imaginary part of the resolution function.

- For non-causal scatterers, reliable reconstruction of both parameters is not possible when using an iterative migration scheme without imposing additional constraints.

- Restricting the iterative update to only velocity perturbations or only attenuation perturbations will remove the ambiguity. 
In Chapter 5, we investigate the influence of the seismic acquisition on this ambiguity. Our conclusions are:

- Sources and receivers on a circle around the scatterer and a cross-well case with a high degree of symmetry remove the ambiguity.

- Acquisitions that are more representative for cases in exploration, mapping both velocity and attenuation perturbations is prone to large errors.

In Chapter 6, we investigate if causality and/or non-linear inversion can alleviate the ambiguity. The chapter includes a noisy example and a more realistic marine setup. We conclude that

- Including causality in the scatterer almost, but not fully, removes the ambiguity, if a sufficiently wide frequency band is used, and if a large number of iterations can be applied.

- Non-causal, non-linear inversion suffers from the same ambiguity, although it less severe. It was not possible to reconstruct the scatterer in a limited amount of iterations.

- Causal, non-linear inversion can reconstruct the velocity and attenuation parameters, although a very large number of iterations is needed.

From this work, our main conclusions are:

- In constant-density visco-acoustic scattering problems, different models produce practically identical data for a surface seismic acquisition.

- When time and computational resources are limited, it is not possible to accurately predict velocity and attenuation perturbations using an iterative migration scheme.

- Only causality can reconstruct the correct location and amplitude of simultaneous velocity and attenuation scatterers from seismic data. 


\subsection{Suggestions for further research}

We would like to conclude with some suggestions for further research. We have seen that an ambiguity occurs when performing linearised inversion with the constant-density visco-acoustic wave equation. We tried to overcome this by changing the acquisition, including causality in the scatterer, and performing nonlinear inversion.

Including causality in the scatterer makes a difference. Basically, it changes the relation between the velocity and attenuation, making it frequency dependent. We did not check other models for attenuation than the Kolsky-Futterman model. It would be interesting to investigate if the ambiguity occurs also for other models. Ursin and Toverud (2002) describe and compare a large number of possible models, e.g., the power law, Kjartansson's model, Müller's model, Amizi's models, the Cole-Cole model, and the standard linear solid model.

We have seen that including causality changes the location of the peak of the gradient, but we did not investigate how this change is related to the reference frequency of causality, peak frequency of the wavelet, or frequency bandwidth, for instance. A properly located peak of the gradient solves part of the task of reconstructing the parameter model. Therefore, it seems useful to further investigate the different frequency components and their influence on the peak location.

Also, we have seen that a more reliable reconstruction of the parameter model may be done by including causality. Hence, further investigation of the frequency dependence may give bounds and limits on the required frequency bandwidth, peak frequency, and reference frequency for this. So far, we noticed, but did not proof, the ambiguity is less well suppressed when the bandwidth decreases. 


\section{Bibliography}

AKI, K. AND Richards, P. G. [2002] Quantitative seismology. University Science Books, Sausalito (CA), USA, 2nd edn. 2, 2.1.3, 2.1.3, 4.2, 5.2, 6.2.1

Barnes, C., Charara, M. and Tsuchiya, T. [2008] Feasibility study for an anisotropic full waveform inversion of cross-well seismic data. Geophysical Prospecting, 56(6), 897-906. 5.1

Berkhout, A. J., Ongkiehong, L., Volker, A. W. F. and Blacquière, G. [2001] Comprehensive assessment of seismic acquisition geometries by focal beams - Part I: Theoretical considerations. Geophysics, 66(3), 911-917. 5.2, 5.3

BEYLKIN, G. [1985] Imaging of discontinuities in the inverse scattering problem by inversion of a causal generalized Radon transform. Journal of Mathematical Physics, 26(1), 99-108. 4.3.5

BEYLKIN, G. AND BURRIDGE, R. [1987] Multiparameter inversion for acoustic and elastic media. SEG 57th Annual Meeting, New Orleans (LA), USA, Expanded Abstracts, 6, 747-749. 2.2.4

BEYLKIN, G. AND BURRIDGe, R. [1990] Linearized inverse scattering problems in acoustics and elasticity. Wave Motion, 12(1), 15-52. 2.2.4, 5.2

Blanch, J. O. and Symes, W. W. [1994] Linear inversion in layered viscoacoustic media using a time-domain method. SEG 64th Annual Meeting, Los Angeles (CA), USA, Expanded Abstracts, 13, 1053-1056. 4.5

Blanch, J. O. and Symes, W. W. [1995] Efficient iterative viscoacoustic linearized inversion. SEG 65th Annual Meeting, Houston (TX), USA, Expanded Abstracts, $14,627-630.4 .5$ 
BLAND, D. R. [1960] The theory of linear viscoelasticity, vol. 10 of International series of monographs on pure and applied mathematics. Pergamon Press, Oxford - London - New York - Paris. 2.1.1

Bleistein, N. And Cohen, J. K. [1977] Nonuniqueness in the inverse source problem in acoustics and electromagnetics. Journal of Mathematical Physics, 18(2), 194-201. 4.5

Bleistein, N., Cohen, J. K. And Stockwell, J. W. [2000] Mathematics of Multidimensional Seismic Imaging, Migration, and Inversion. Springer-Verlag, New York (NY), USA. 5.2

Bohm, D. And Weinstein, M. [1948] The self-oscillations of a charged particle. Physical Review, 74(12), 1789-1798. 4.5

van den Bos, A. [1994] Complex gradient and Hessian. IEE Proceedings - Vision, Image, and Signal Processing, 141(6), 380-382. 3.2.3

Byrd, R. H., Lu, P., Nocedal, J. And Zhu, C. Y. [1995] A limited memory algorithm for bound constrained optimization. SIAM Journal on Scientific Computing, 16(5), 1190-1208. 6.2.3

Causse, E., Mittet, R. and Ursin, B. [1999] Preconditioning offull-waveform inversion in viscoacoustic media. Geophysics, 64(1), 130-145. 1.2, 3.1, 5.1

Chavent, G. and Plessix, R.-E. [1999] An optimal true-amplitude least-squares prestack depth-migration operator. Geophysics, 64(2), 508-515. 4.3.5, 5.2

Chen, J. And Schuster, G. T. [1999] Resolution limits of migrated images. Geophysics, 64(4), 1046-1053. 5.2, 5.3

Crase, E., Pica, A., Noble, M., McDonald, J. and Tarantola, A. [1990] Robust elastic nonlinear waveform inversion: Application to real data. Geophysics, 55(5), 527-538. 5.1

Crase, E., Wideman, C., Noble, M. and Tarantola, A. [1992] Nonlinear elastic waveform inversion of land seismic reflection data. Journal of Geophysical Research, 97(B4), 4685-4703. 5.1

Devaney, A. J. [1978] Nonuniqueness in the inverse scattering problem. Journal of Mathematical Physics, 19(7), 1526-1531. 4.5

Devaney, A. J. [1984] Geophysical diffraction tomography. IEEE Transactions on 
Geoscience and Remote Sensing, GE-22(1), 3-13. 5.2

Devaney, A. J. [2004] Nonradiating surface sources. Journal of the Optical Society of America A, 21(11), 2216-2222. 4.5

Devaney, A. J. and Marengo, E. A. [1998] A method for specifying non-radiating, monochromatic, scalar sources and their fields. Pure and Applied Optics, 7(5), 12131220. 4.5

Devaney, A. J. and Sherman, G. C. [1982] Nonuniqueness in inverse source and scattering problems. IEEE Transactions on Antennas and Propagation, 30(5), 10341037. 4.5

Docherty, P. [1991] A brief comparison of some Kirchhoff integral formulas for migration and inversion. Geophysics, 56(8), 1164-1169. 4.3.5, 5.2

Futterman, W. I. [1962] Dispersive body waves. Journal of Geophysical Research, 67(13), 5279-5291. 2.1.3

Gélis, C., Virieux, J. and Grandjean, G. [2007] Two-dimensional elastic full waveform inversion using Born and Rytov formulations in the frequency domain. Geophysical Journal International, 168(2), 605-633. 2.2.4, 5.1

George, A. ANd Liv, J. W. H. [1981] Computer solution of large sparse positive definite systems. Prentice-Hall, Englewood Cliffs (NJ), USA. 6.2.3

Gjøystdal, H., Iversen, E., Laurain, R., Lecomte, I., Vinje, V. and Åstebøl, K. [2002] Review of ray theory applications in modelling and imaging of seismic data. Studia Geophysica et Geodaetica, 46(2), 113-164. 5.2

Goedecke, G. H. [1964] Classically radiationless motions and possible implications for quantum theory. Physical Review, 135(1B), B281-B288. 4.5

Gradshteyn, I. S. And Ryzhiк, I. M. [1965] Table of integrals, series, and products. Academic Press, New York (NY), USA, 4th edn. 4.3.4

GraY, S. [1997] True-amplitude seismic migration: a comparison of three approaches. Geophysics, 62(3), 929-936. 4.3.5, 5.2

Guitton, A. [2004] Amplitude and kinematic corrections of migrated images for nonunitary imaging operators. Geophysics, 69(4), 1017-1024. 5.2

HaK, B. And Mulder, W. A. [2008a] Preconditioning for linearised inversion of attenuation and velocity perturbations. 70th EAGE Conference \& Exhibition, Rome, Italy, 
Extended Abstracts, H002. 3.1, 4.5, 5.1

HaK, B. And Mulder, W. A. [2008b] Preconditioning for linearized inversion of attenuation and velocity perturbations. SEG 78th Annual Meeting, Las Vegas (NV), USA, Expanded Abstracts, 27, 2036-2040. 3.1, 5.1

HaK, B. ANd Mulder, W. A. [2010a] Migration for velocity and attenuation perturbations. Geophysical Prospecting, 58(6), 939-952. 1.3, 6.1, 6.2.1, 6.3, 6.3

HaK, B. ANd Mulder, W. A. [2010b] Seismic attenuation imaging with causality. Geophysical Journal International. (accepted). 1.3

Harari, I. ANd Turkel, E. [1995] Accurate finite difference methods for time-harmonic wave propagation. Journal of Computational Physics, 119(2), 252-270. 6.2.3

Hicks, G. J. And Pratt, R. G. [2001] Reflection waveform inversion using local descent methods: estimating attenuation and velocity over a gas-sand deposit. Geophysics, 66(2), 598-612. 1.2, 3.1, 5.1, 5.5, 6.1

Hoenders, B. J. [1997] Existence of invisible nonscattering objects and nonradiating sources. Journal of the Optical Society of America A, 14(1), 262-266. 4.5

van Houten, E. E. W., Paulsen, K. D., Miga, M. I., Kennedy, F. E. and Weaver, J. B. [1999] An overlapping subzone technique for MR-based elastic property reconstruction. Magnetic Resonance in Medicine, 42(4), 779-786. 2.2.4, 5.2

Innanen, K. A. And Weglein, A. B. [2007] On the construction of an absorptivedispersive medium model via direct linear inversion of reflected seismic primaries. Inverse Problems, 23(6), 2289-2310. 4.5, 5.1, 6.1, 6.3

Jin, S., Madariaga, R., Virieux, J. and Lambaré, G. [1992] Two-dimensional asymptotic iterative elastic inversion. Geophysical Journal International, 108(2), 575-588. 5.1

Jo, C.-H., Shin, C. And Suh, J. H. [1996] An optimal 9-point, finite-difference, frequencyspace, 2-D scalar wave extrapolator. Geophysics, 61(2), 529-537. 6.2.3

Kamei, R. and Pratt, R. G. [2008] Waveform tomography strategies for imaging attenuation structure with cross-hole data. 70th EAGE Conference \& Exhibition, Rome, Italy, Extended Abstracts, F019. 6.1

Kennett, B. L. N. ANd SAmbridge, M. [1998] Inversion for multiple parameter classes. Geophysical Journal International, 135(1), 304-306. 5.1 
Kennett, B. L. N., Sambridge, M. S. and Williamson, P. R. [1988] Subspace methods for large inverse problems with multiple parameter classes. Geophysical Journal International, 94(2), 237-247. 5.1

Kolsky, H. [1956] The propagation of stress pulses in viscoelastic solids. Philosophical Magazine, 1(8), 693-710. 2.1.3

ten Kroode, A. P. E., Smit, D.-J. And Verdel, A. R. [1998] A microlocal analysis of migration. Wave Motion, 28(2), 149-172. 4.3.5, 5.2

Kuvshinov, B. N. And Mulder, W. A. [2006] The exact solution of the time-harmonic wave equation for a linear velocity profile. Geophysical Journal International, 167(2), 659-662. 5.4.3, 5.4.3

LAILly, P. [1983] The seismic inverse problem as a sequence of before stack migrations. In: Proceedings of the international conference on inverse scattering: Theory and applications, edited by J. B. Bednar, R. Redner, E. Robinson and A. Weglein, SIAM, Philadelphia (PA), USA, 206-220. 1, 2.2, 3.1, 5.1, 5.2

Lambaré, G., Virieux, J., Madariaga, R. and Jin, S. [1992] Iterative asymptotic inversion in the acoustic approximation. Geophysics, 57(9), 1138-1154. 3.1, 5.1

Lines, L., Vasheghani, F. and Treitel, S. [2008] Reflections on Q. CSEG Recorder, 33(10), 36-38. 1.2

Marfurt, K. J. And Shin, C. S. [1989] The future of iterative modeling in geophysical exploration. In: Supercomputers in seismic exploration, edited by E. Eisner, Pergamon Press, Oxford, UK. 6.2.3

Mora, P. [1987] Nonlinear two-dimensional elastic inversion of multioffset seismic data. Geophysics, 52(9), 1211-1228. 5.1

Mora, P. [1988] Elastic wave-field inversion of reflection and transmission data. Geophysics, 53(6), 750-759. 5.1

Mora, P. [1989] Inversion = migration + tomography. Geophysics, 54(12), 1575-1586. 5.1

Mulder, W. A. ANd HaK, B. [2009] An ambiguity in attenuation scattering imaging. Geophysical Journal International, 178(3), 1614-1624. 1.3, 5.1, 5.3, 5.5, 5.A, 6.1, 6.2.1

Mulder, W. A. And Plessix, R.-E. [2004] A comparison between one-way and two-way 
wave-equation migration. Geophysics, 69(6), 1491-1504. 1.1, 4.1, 4.4, 5.1, 5.2, 6.2.3, 6.3

Mulder, W. A. And Plessix, R.-E. [2008] Exploring some issues in acoustic full waveform inversion. Geophysical Prospecting, 56(6), 827-841. 5.1, 5.4.3

Nemeth, T., Wu, C. And Schuster, G. T. [1999] Least-squares migration of incomplete reflection data. Geophysics, 64(1), 208-221. 3.1, 5.1

O'Connell, R. J. and Budiansky, B. [1978] Measures of dissipation in viscoelastic media. Geophysical Research Letters, 5(1), 5-8. 2.1.1

Operto, S., Ravaut, C., Improta, L., Virieux, J., Herrero, A. and Dell'Aversana, P. [2004] Quantitative imaging of complex structures from dense wide-aperture seismic data by multi-scale traveltime and waveform inversions: a case study. Geophysical Prospecting, 52(6), 625-651. 5.1

Østmo, S., Mulder, W. A. And Plessix, R.-E. [2002] Finite-difference iterative migration by linearized waveform inversion in the frequency domain. SEG 72nd Annual Meeting, Salt Lake City (UT), USA, Expanded Abstracts, 21, 1384-1387. 3.1, 4.1, $4.4,5.1,5.2,6.3$

Plessix, R.-E. [2006] A review of the adjoint-state method for computing the gradient of a functional with geophysical applications. Geophysical Journal International, 167(2), 495-503. 6.A

Plessix, R.-E. And Mulder, W. A. [2004] Frequency-domain finite-difference amplitudepreserving migration. Geophysical Journal International, 157(3), 975-987. 2.2.4, $3.1,4.1,4.3 .5,4.4,5.1,5.2$

PRATT, R. G. [1999] Seismic waveform inversion in the frequency domain, Part 1: theory and verification in a physical scale model. Geophysics, 64(3), 888-901. 5.1

Pratt, R. G., Shin, C. S. And Hicks, G. J. [1998] Gauss-Newton and full Newton methods in frequency-space seismic waveform inversion. Geophysical Journal International, 133(2), 341-362. 2.2.4, 5.1, 5.2

RAO, Y. AND WANG, Y. H. [2008] The strategies for attenuation inversion with waveform tomography. 70th EAGE Conference \& Exhibition, Rome, Italy, Extended Abstracts, H006. 3.1, 5.5

Ribodetti, A., Gaffet, S., Operto, S., Virieux, J. and Saracco, G. [2004] Asymptotic waveform inversion for unbiased velocity and attenuation measurements: Numerical 
tests and application for Vesuvius lava sample analysis. Geophysical Journal International, 158(1), 353-371.

Ribodetti, A. And Hanyga, A. [2004] Some effects of the memory kernel singularity on wave propagation and inversion in viscoelastic media - ii. inversion. Geophysical Journal International, 157(2), 426-442. 6.1, 6.3

Ribodetti, A., Operto, S., Virieux, J., Lambaré, G., Valéro, H. P. and Gibert, D. [2000] Asymptotic viscoacoustic diffraction tomography of ultrasonic laboratory data: a tool for rock properties analysis. Geophysical Journal International, 140(2), 324-340. 5.4.1, 6.1

Ribodetti, A., Plessix, R.-E., Operto, S. and Virieux, J. M. [2007] Viscoacoustic frequency-domain full-waveform inversion - application to numerical VSP data. 69th EAGE Conference \& Exhibition, London, UK, Extended Abstracts, C029. 3.1, 6.1

Ribodetti, A. And Virieux, J. [1998] Asymptotic theory for imaging the attenuation factor $Q$. Geophysics, 63(5), 1767-1778. 1.2, 4.5, 5.1, 6.1

Ribodetti, A., Virieux, J. And Durand, S. [1995] Asymptotic theory for viscoacoustic seismic imaging. SEG 65th Annual Meeting, Houston (TX), USA, Expanded Abstracts, 14, 631-634. 4.5, 5.1

RicketT, J. E. [2003] Illumination-based normalization for wave-equation depth migration. Geophysics, 68(4), 1371-1379. 5.2

Royle, G. T. And Singh, S. C. [2008] Viscoelastic modeling and full waveform inversion in attenuating media. 70th EAGE Conference \& Exhibition, Rome, Italy, Workshop WO11, 121-125. 5.1

VON SEggern, D. [1991] Spatial resolution of acoustic imaging with the Born approximation. Geophysics, 56(8), 1185-1202. 5.2

SHeriff, R. E. [2002] Encyclopedic dictionary of applied geophysics. Society of Exploration Geophysicists, Tulsa (OK), USA, 4th edn. 1

Shewchuк, J. R. [1994] An introduction to the conjugate gradient method without the agonizing pain. Notes, Carnegie Mellon University, Pittsburgh (PA), USA. 3.5

SHIN, C., JANG, S. AND Min, D.-J. [2001] Improved amplitude preservation for prestack depth migration by inverse scattering theory. Geophysical Prospecting, 49(5), 592606. 2.2.4, 4.3.5, 5.1 
Shipp, R. M. AND Singh, S. C. [2002] Two-dimensional full wavefield inversion of wideaperture marine seismic streamer data. Geophysical Journal International, 151(2), 325-344. 5.1

Sirgue, L. And Pratt, R. G. [2004] Efficient waveform inversion and imaging: a strategy for selecting temporal frequencies. Geophysics, 69(1), 231-248. 4.3.4

Smithyman, B. R., Pratt, R. G., Hayles, J. G. and Wittebolle, R. J. [2009] Detecting near-surface objects with seismic waveform tomography. Geophysics, 74(6), WCC119WCC127. 6.1

Symes, W. W. [2008] Approximate linearized inversion by optimal scaling of prestack depth migration. Geophysics, 73(2), R23-R35. 5.2

TARANTOla, A. [1984] Inversion of seismic reflection data in the acoustic approximation. Geophysics, 49(8), 1259-1266. 1, 2.2, 3.1, 5.1, 5.2

Tarantola, A. [1986] A strategy for nonlinear elastic inversion of seismic reflection data. Geophysics, 51(10), 1893-1903. 5.1

Tarantola, A. [1988] Theoretical background for the inversion of seismic waveforms, including elasticity and attenuation. Pure and Applied Geophysics, 128(1/2), 365399. 3.1

Titchmarsh, E. C. [1948] Introduction to the theory of Fourier integrals. Clarendon Press, Oxford, UK, 2nd edn. 2.1.3

Toksöz, M. N. And Johnston, D. [1981] Seismic wave attenuation. Society of Exploration Geophysicists, Tulsa (OK), USA, 2nd edn. 2.1.3

Toxopeus, G., Petersen, S., Thorbecke, J. and Wapenaar, K. [2004] Simulating high resolution inversion: decomposing the resolution function. SEG 74th Annual Meeting, Denver (CO), USA, Expanded Abstracts, 23, 1798-1801. 5.2

Toxopeus, G., Thorbecke, J., Wapenaar, K., Petersen, S., Slob, E. and Fokkema, J. [2008] Simulating migrated and inverted seismic data by filtering a geologic model. Geophysics, 73(2), T1-T10. 5.2

Ursin, B. AND Toverud, T. [2002] Comparison of seismic dispersion and attenuation models. Studia Geophysica et Geodaetica, 46(2), 293-320. 7.2

Vasheghani, F. And Lines, L. R. [2009] Viscosity and $Q$ in heavy-oil reservoir characterization. The Leading Edge, 28(7), 856-860. 1.1, 1.1, 1.2, 3.1, 5.1 
WAPENAAR, C. P. A. [1997] 3-D migration of cross-spread data: resolution and amplitude aspects. Geophysics, 62(4), 1220-1225. 5.2

Wu, R.-S. And Toksöz, M. N. [1987] Diffraction tomography and multisource holography applied to seismic imaging. Geophysics, 52(1), 11-25. 5.3

Yoon, K., Marfurt, K. J. And StARr, W. [2004] Challenges in reverse-time migration. SEG 74th Annual Meeting, Denver (CO), USA, Expanded Abstracts, 23, 10571060. 1.1 


\section{Acknowledgements}

Any successful PhD project is built on the support of a group of people. Here, I like to acknowledge and thank all these people, with my apologies if I am not complete.

First of all, I would like to thank my promotor Wim Mulder for all his time and effort, and his insight in making this thesis to the scientific work it is now. Wim, thank you. Also, I would really like to thank my committee members for the time they made available for my defense and for giving their useful comments.

Finally, I like to thank all my (former) colleagues at TU Delft and at Shell, where I would like to specially mention Christiaan, Mariandl, and Tristan. Thank you all for the coffee breaks, conferences, sports, and discussions, but basically for making the working days and office life enjoyable.

I would really like to thank my family and friends. Most of you probably didn't have a direct impact on my PhD, but believe me, all of you helped me in some way; weekends back home, a holiday in Hungary, hiking in the mountains, birthday parties, bachelor parties, sinterklaas celebrations, randstad drinks, game-weekends, and even the occasional barbecue. Thank you all. I would specially like to thank Dasha. You probably don't even realise how much you helped me, but you did. Thank you so much dear Dasha. I would also like to thank mam and pap for their unconditional support and love. That is what a son needs every day.

- Bobby - 


\section{Curriculum Vitae}

\section{Personal}

Name

Bobby Hak.

Born

$11^{\text {th }}$ May 1979, Laren, The Netherlands.

Nationality

Dutch.

\section{Education}

1991 - 1998 HAVO \& VWO, Christelijke Scholengemeenschap Dingstede, Meppel, The Netherlands.

1998 - 2005 MSc Mathematical Engineering, University of Groningen, Groningen, The Netherlands.

2005 - 2010 PhD Applied Geophysics and Petrophysics, Delft University of Technology, Delft, The Netherlands.

\section{Work}

2010 - present Project Geoscientist, Fugro Jason,

Leidschendam, The Netherlands. 


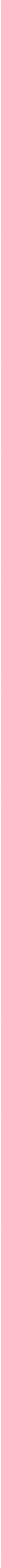

